\title{
Fully Bayesian Modeling for fMRI Group Analysis
}

\author{
Johnatan Cardona Jiménez \\ DOCTORAL THESIS SUBMITTED AT THE INSTITUTE \\ OF MATHEMATICS AND STATISTICS \\ OF THE UNIVERSITY OF SÃO PAUlO \\ IN FULFILMENT OF THE REQUIREMENTS \\ FOR THE DEGREE \\ OF \\ Doctor of Philosophy (PhD) \\ Program: Statistics \\ Supervisor: Dr. Carlos Alberto de Bragança Pereira
}

São Paulo, October 14, 2019 


\section{Resumo}

Jiménez, J.C. Modelagem totalmente Bayesiana para análise de grupos com dados de fMRI. Tese (Doutorado) - Instituto de Matemática e Estatística, Universidade de São Paulo, São Paulo, 2019.

Imagens de ressonância magnética funcional ou MRI funcional (fMRI) é uma forma não invasiva de avaliar a atividade cerebral através da deteç̧ão de mudanças relacionadas ao fluxo sanguíneo. Nesta tese propomos uma modelagem Bayesiana completa para analisar dados de fMRI para o caso individual e em grupos. Para a etapa individual, usamos a Modelo Linear Dinâmico Matriz-Variado (MLDMV), onde a dependência temporal é modelada através dos parâmetros de estado e a dependência espacial é modelada apenas localmente, considerando os vizinhos mais próximos de cada voxel. Para a fase de grupos, a partir da distribuição posterior dos parâmetros de estado obtidos no estágio individual criamos uma nova distribuição posterior que representam as crenças atualizadas para a análise de grupo. Como a distribuição posterior dos parâmetros de estado é indexada pelo tempo t, propomos três opções para algoritmos que permitem amostrar curvas estimadas dos parâmetros de estado e calcular probabilidades posteriores para avaliar a ativação cerebral para os estágios individual e de grupo. Ilustramos nosso método por meio de dois exemplos práticos e oferecemos uma avaliação usando dados reais de resting-state para calcular taxas empíricas de ativações falso-positivas. Finalmente, disponibilizamos um pacote de R (BayesDLMfMRI) para executar análises de dados de fMRI baseada em tarefas para etapas individuais e de grupo usandoo método proposto nesta tese.

Palavras-chave: Modelos lineares dinâmicos, Análise Bayesiana, Integração de Monte Carlo. 


\section{Abstract}

Jiménez, J.C. Fully Bayesian Modeling for fMRI Group Analysis. Thesis (PhD) - Institute of Mathematics and Statistics, University of São Paulo, 2019.

Functional magnetic resonance imaging or functional MRI (fMRI) is a non-invasive way to assess brain activity by detecting changes associated with blood flow. In this thesis, we propose a fully Bayesian procedure to analyze fMRI data for individual and group stages. For the individual stage, we use a Matrix-Variate Dynamic Linear Model (MDLM), where the temporal dependence is modeled through the state parameters and the spatial dependence is modeled only locally, taking the nearest neighbors of each voxel location. For the group stage, we take advantage of the posterior distribution of the state parameters obtained at the individual stage and create a new posterior distribution that represents the updated beliefs for the group analysis. Since the posterior distribution for the state parameters is indexed by the time $t$, we propose three options for algorithms that allow on-line estimated curves for the state parameters to be drawn and posterior probabilities to be computed in order to assess brain activation for both individual and group stages. We illustrate our method through two practical examples and offer an assessment using real resting-state data to compute empirical false-positive brain activation rates. Finally, we make available the $\mathbf{R}$ package BayesDLMfMRI to perform task-based fMRI data analysis for individual and group stages using the method proposed in this thesis.

Keywords: Dynamic linear models, functional MRI, Bayesian analysis, Monte Carlo integration. 


\section{Contents}

1 Introduction $\quad 1$

1.0.1 What is a Magnetic Resonance Image (MRI)? . . . . . . . . . . . . . . . . . . 2

1.0.2 What is an fMRI experiment? . . . . . . . . . . . . . . . 3

1.0.3 The blood-oxygenation-level dependent (BOLD) signal and the hemodynamic response function $(\mathrm{HRF}) \ldots \ldots \ldots \ldots \ldots$

1.0.4 The observed and expected BOLD signal . . . . . . . . . . . . 5

1.1 Statistical modeling of the BOLD signal . . . . . . . . . . . . . . 6

1.1 .1 Group analysis . . . . . . . . . . . . . . . . . 7

1.1.2 About software packages for fMRI analysis . . . . . . . . . . . 8

2 Paper 1: Assessing Dynamic Effects on a Bayesian Matrix-Variate Dynamic Linear Model: an Application to fMRI data Analysis

3 Paper 2: fMRI group analysis based on outputs from Matrix-Variate Dynamic Linear Models

4 Paper 3: BayesDLMfMRI: Bayesian Matrix-Variate Dynamic Linear Models for fRMI modeling in $\mathbf{R}$

References 
viii CONTENTS 


\section{Chapter 1}

\section{Introduction}

Magnetic resonance imaging (MRI) is a non-invasive technique that is used to create elaborate anatomical images of the human body. Specifically, this technique can be used to obtain detailed brain images that can help to identify different types of tissue such as white matter and gray matter, and can also be used to diagnose aneurysms and tumors. Another important facet of this technique is that it can be used to visualize dynamic or functional activity in the brain. Functional Magnetic Resonance Imaging (fMRI) can be described as a generalization of the MRI technique, where the focus is not just one high-resolution image of the brain, but rather a sequence of low-resolution images that allows for identification, at least in an indirect way, of neuronal activity through the blood-oxygen-level dependent (BOLD) contrast. In this regard, statistical methods are very useful for analyzing the post-processed data that compose the sequence of images obtained by fMRI experiment. For instance, the most popular statistical model used to assess brain reaction due to an external stimulus is the normal regression linear model, usually known as General Linear Model (GLM) in the fMRI literature [Beckmann et al., 2003]. This type of modeling assumes spatiotemporal independence among the data, an unrealistic assumption that can lead to incorrect inference ([Eklund et al., 2012], Eklund et al. [2016]). However, more sophisticated statistical methods for fMRI data analysis which take into account the complex temporal and spatial dependence (or at least one of them) have already been published on statistical and neuroscience journals in the last two decades (some recent and relevant references: [Zhang et al., 2016], [Eklund et al., 2017], [Bezener et al., 2018], [Yu et al., 2018]). The reviews by Bowman [2014] and Zhang et al. [2015] offer a general insight on statistical methods for brain imaging analysis and a complete review of Bayesian methods for task-based fMRI data analysis.

In spite of the availability of different statistical tools to address the problem of modeling fMRI data, the GLM seems to be the more common method used until now. There are some reasons for this: just a few options of software implementations or packages with alternative methods; the complexity related to the implementation of Bayesian methods for non-statisticians or novice users; the most common packages for fMRI data analysis such as FSL [Jenkinson et al., 2012] and SPM [Penny et al., 2011] lead the users to run their most common tools (GLM being one of them). Another issue that makes the modeling of this type of data into a huge challenge is the vast amounts of data usually contained within an fMRI array. For instance, in one of the practical examples shown in the next chapters, the "voice localizer" experiment [Pernet et al., 2015], its data can be represented in an array of dimensions $x=91 \times y=109 \times z=91 \times t=310 \times N_{s}=207$, where the vector $[x, y, z]$ represents the position on the brain image, $t$ is the number on the sequences of brain images and $N_{S}$ is the number of subjects or participants in the study.

In this work, we propose Bayesian modeling for task-based fMRI data using Matrix-Variate Dynamic Linear Models (MDLM). Our aim with this type of modeling is to take advantage of the flexibility offered by the general theory of Dynamic Linear Models (DLM) [West \& Harrison, 1997] to easily account for the temporal dependence present is this type of data. Furthermore, we use the MDLM proposed by Quintana [1985] to jointly model groups of temporal series from clusters of voxels (see figure (1.1)) and therefore (at least locally) take into account the spatial dependence. 
Some of the goals when modeling task-based fMRI data are to assess brain activation either on a single individual or on groups of subjects as well as compare the strength of brain activation between groups (e.g. cases vs controls). Hence, in the context of the modeling proposed in this work the assessment of brain activation is performed through the state parameter, for which we propose three options of algorithms to sample on-line trajectories of that dynamic effect and compute a measure of evidence for brain activation via Monte Carlo. To make this method available for potentially interested users, we offer an $\mathrm{R}$ package called BayesDLMfMRI, which allows assessing brain activation at individual and group stages.

\section{Outline}

In the remaining part of this chapter we introduce basic concepts on fMRI experiments, going through the explanation of what is a Magnetic Resonance Image (MRI) and the acquisition process in a functional Magnetic Resonance Image (fMRI) experiment. We also explain the important concepts of blood-oxygen-level dependet (BOLD) contrast imaging and the hemodynamic response function. We then introduce the construction of the expected BOLD response, the definition of GLM, and finally, we mention some of the most popular software packages for fMRI data analysis and the models implemeted on them. In chapter 2, we present an article where our method for assessing dynamic effects or (in the context of task-based fMRI data analysis) detecting brain activation is introduced. The method is illustrated through two practical examples and assessments using simulated and real resting-state fMRI data are performed in order to evaluate the capability of the method related to detections of real and false brain activations. In chapter 3, an article with the extension of our method for the group case is presented and finally in chapter 4, we introduce the $\mathbf{R}$ package BayesDLMfMRI, where the proposal presented here has been implemented.

\subsubsection{What is a Magnetic Resonance Image (MRI)?}

We now give a brief description about the composition of a Magnetic Resonance Image (MRI). This can be important in order to understand some basic characteristics of the random variables we are going to deal with. It is worthwhile pointing out that some preprocessing is necessary for these types of images in order to take raw data from the scanner and prepare them for statistical analysis. Some of the steps usually applied in the image preprocessing are motion correction, slice timing correction, spatial filtering, intensity normalization, and temporal filtering. Here, we do not explain those in detail, however more information can be found here: Poldrack et al. [2011]. An MRI can be viewed as a matrix of numbers that correspond to spatial locations. When we view an image, we do so by representing the numbers in the image in terms of grayscale values and each element in the image is called as a voxel, which is the three-dimensional analog to a pixel. See figure 1.1 for a visual description.
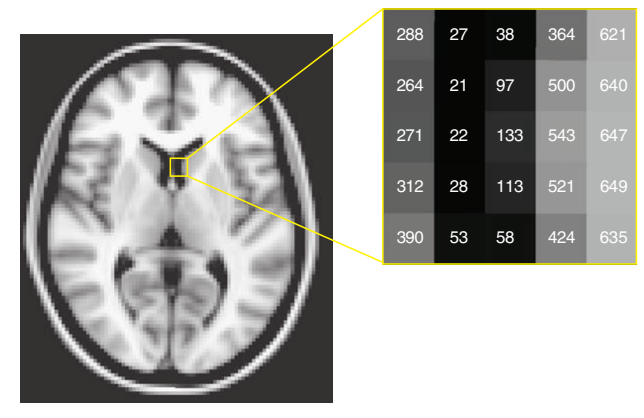

Figure 1.1: An image as a graphical representation of a matrix. The grayscale values in the image on the left correspond to numbers, shown for a specific set of voxels in the closeup section on the right ([Poldrack et al., 2011). 


\subsubsection{What is an fMRI experiment?}

An MRI of the brain can be describeded as a high-resolution picture which is obtained from an MRI scanner (See the right panel in figure 1.2)). Thus, in order to take it, the individual has to lie in the scanner for a period of time, usually 5 minutes, in a rest state until the entire brain is scanned. This type of image can help to identify different types of tissue, such as white matter and gray matter, and can also be used to diagnose aneurysms and tumors. On the other hand, an fMRI experiment is a process where the individual can also stay lying in the scanner for the same period of time -five minutes- but up to 100 low-resolution images can be obtained. See the right side in the left panel in figure 1.2). In an fMRI experiment, the individual can either receive a sequence of stimuli according to a specific design (task-based fMRI experiment) or just stay in a resting position without any external stimulation (resting-state fMRI experiment).

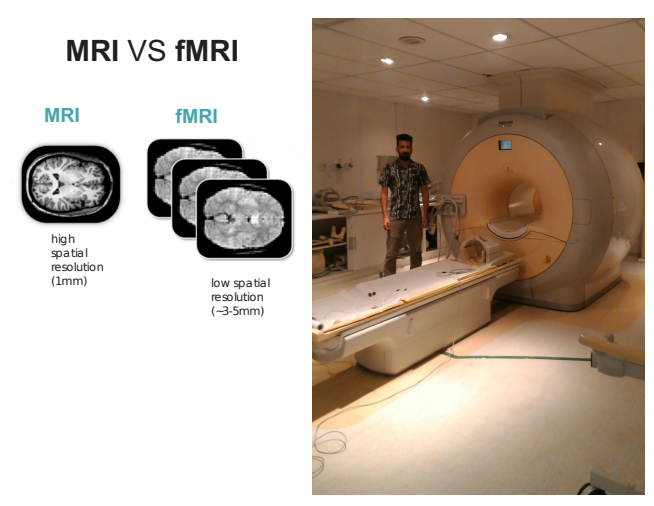

Figure 1.2: Left panel: Diferences between MRI and fMRI images. Right panel: The MRI scanner at the Institute of Radiology of the University of São Paulo.

The types of stimuli presented to the subject in the scanner in an task-based fMRI experiment depend on the interests of the researcher, and usually include sensory, visual and/or auditory stimuli. There are three different types of experimental designs or paradigms that are usually applied in a task-based fMRI experiment: block design, event-related design, and a composition of the two. In a block design, a stimulus is presented in a continuous way, say, in intervals with durations of 20 to 30 seconds, with sequences of resting intervals where the stimulus is not presented. See panel (a) of figure 1.3. On the other hand, in an event-related design, a stimulus of a short duration -2 seconds or less- is presented, followed by more long periods of rest. In this kind of paradigm, the stimuli can be presented in a random or fixed way. See panel (c) of figure 1.3. In general, block designs are statistically powerful and straighforward to analyze. The disadvantage of this design is that the participant may start to predict or anticipate the task. In contrast, event-related designs can be more easily randomized because of the short stimulus duration, and allows for stimulus events from different experimental conditions to be displayed in one run, something that is not possible with block designs. The main disadvantage of this type of deign is the weak signal-to-noise ratio (SNR), leading to a loss of statistical power. In general, the paradigm chosen will depend on the goal of the experiment (Kashou [2014]). 
(a) Block design

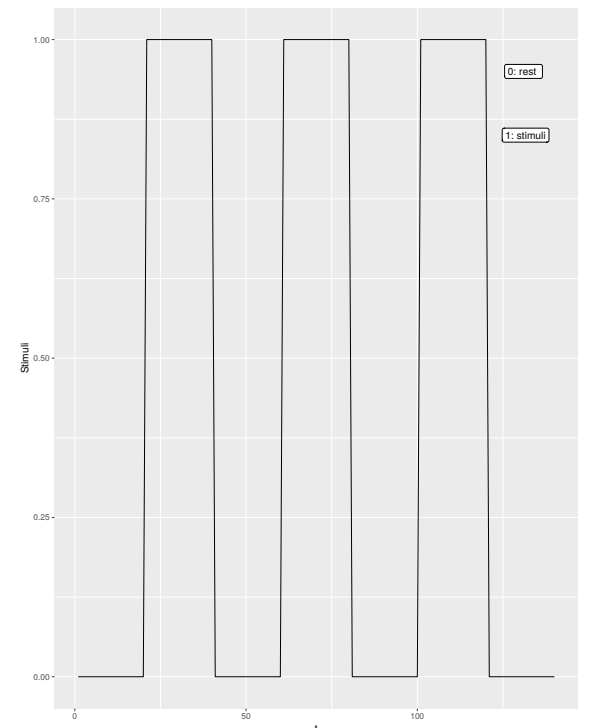

(c) Event related design

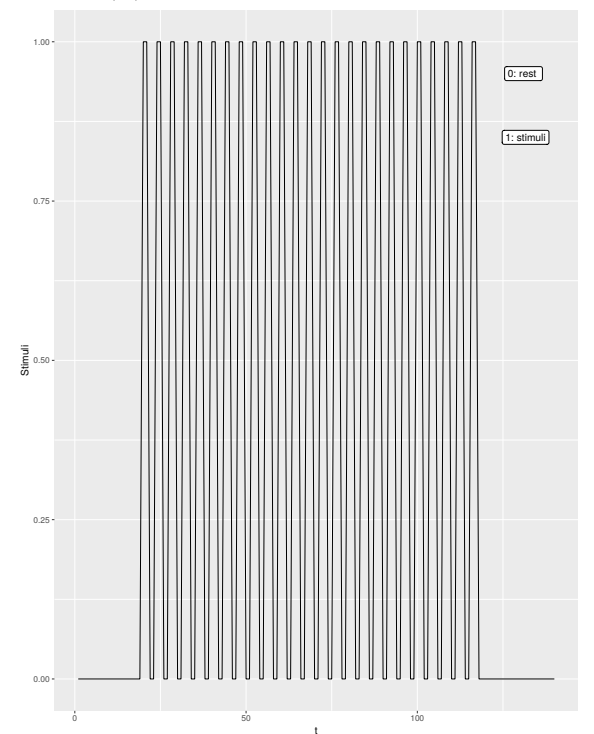

(b) Mixed block/event related design

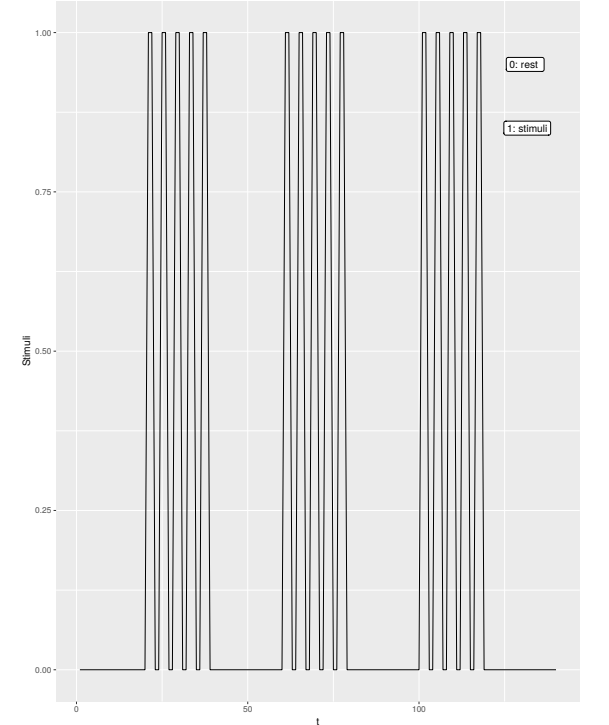

(d) randomized event related design

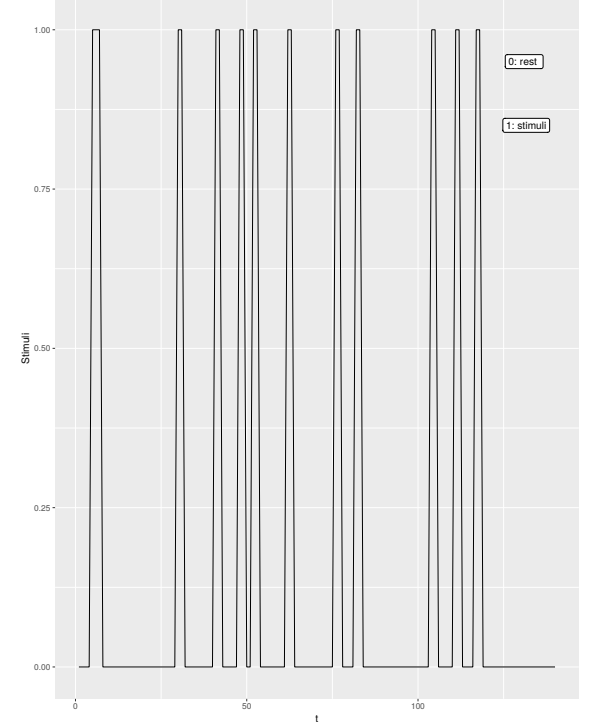

Figure 1.3: Some alternatives of designs broadly used in fMRI experiments.

\subsubsection{The blood-oxygenation-level dependent (BOLD) signal and the hemody- namic response function (HRF)}

Above, we give a description of an MRI scan in terms of numeric information. Now it is explained how the neural activation, at least in an indirect way, can be pictured in a sequence of MRI scans or in an fMRI experiment. In order to do so, we refer to Poldrack et al. [2011]: "The most common method of fMRI takes advantage of the fact that when neurons in the brain become active, the amount of blood flowing through that area is increased. This phenomenon has been known for more than 100 years, though the mechanisms that cause it remain only partly understood. What is particularly interesting is that the amount of blood that is sent to the area is more than is needed to replenish the oxygen that is used by the activity of the cells. Thus, the activity-related increase in blood flow caused by neuronal activity leads to a relative surplus in local blood oxygen. The signal measured in fMRI depends on this change in oxygenation and is referred to as the 
blood-oxygenation-level dependent, or BOLD signal." In figure 1.4 we can see the shape of the hemodynamic response function (HRF), which is the picture over time of the change of the BOLD signal. Thus, when the neuronal activity increases in a specific region of the brain, the BOLD signal also does, and the electromagnetic field generated by the MRI scanner captures those changes, allowing the phenomenon to be transformed into numeric information.

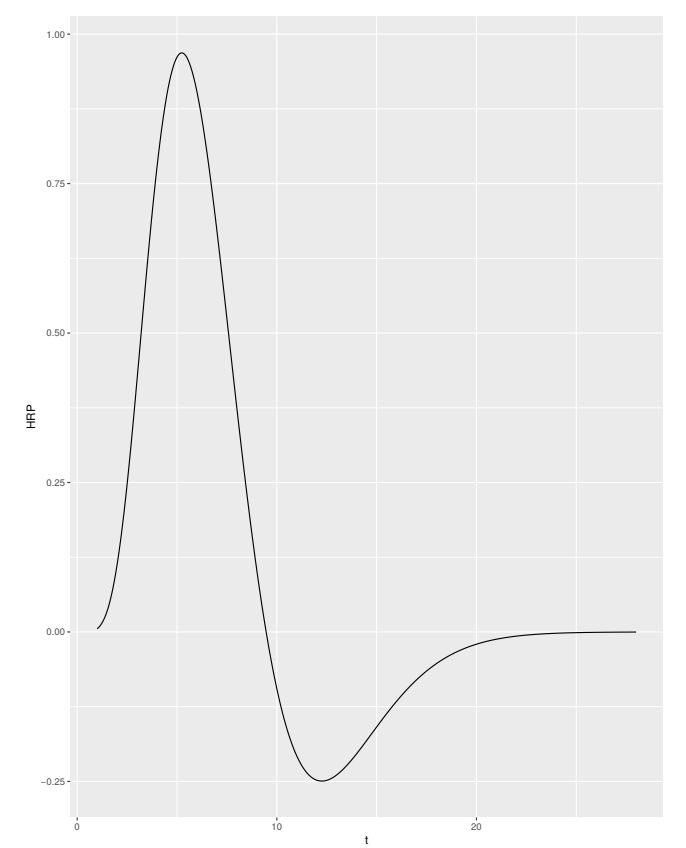

Figure 1.4: Hemodynamic Response Function:h.

\subsubsection{The observed and expected BOLD signal}

One of the main objectives in a task-based fMRI experiment is to identify a brain reaction in response to some controlled external stimuli, in other words, to look for changes in the BOLD signal related to some experimental manipulation. In order to do that, one can use any of the designs mentioned above. For instance, in figure 1.5 (left panel), we can see the block design related to a sequence of stimuli and its resulting observed BOLD signal for a particular voxel. In that case, the goal is to find the voxel time series (i.e., the observed BOLD signals) that matches this pattern (block design) as they do. However, we can see that the BOLD signal does not follow the block stimuli very well, due to slow physiological response. Hence, in order to correct that delay, the stimulus time series $f$ (such as in figure 1.5, left panel) is blended with an HRF, $h$ (such as figure 1.4), creating a shape that more closely represents the shape of the BOLD response, which is usually called "expected BOLD response." See figure 1.5 right panel. To find out more about the BOLD response and its properties refer to Poldrack et al. [2011]. The computation of the expected BOLD response is given by the convolution:

$$
x(t)=(h * f)(t)=\int h(\tau) f(t-\tau) d \tau
$$



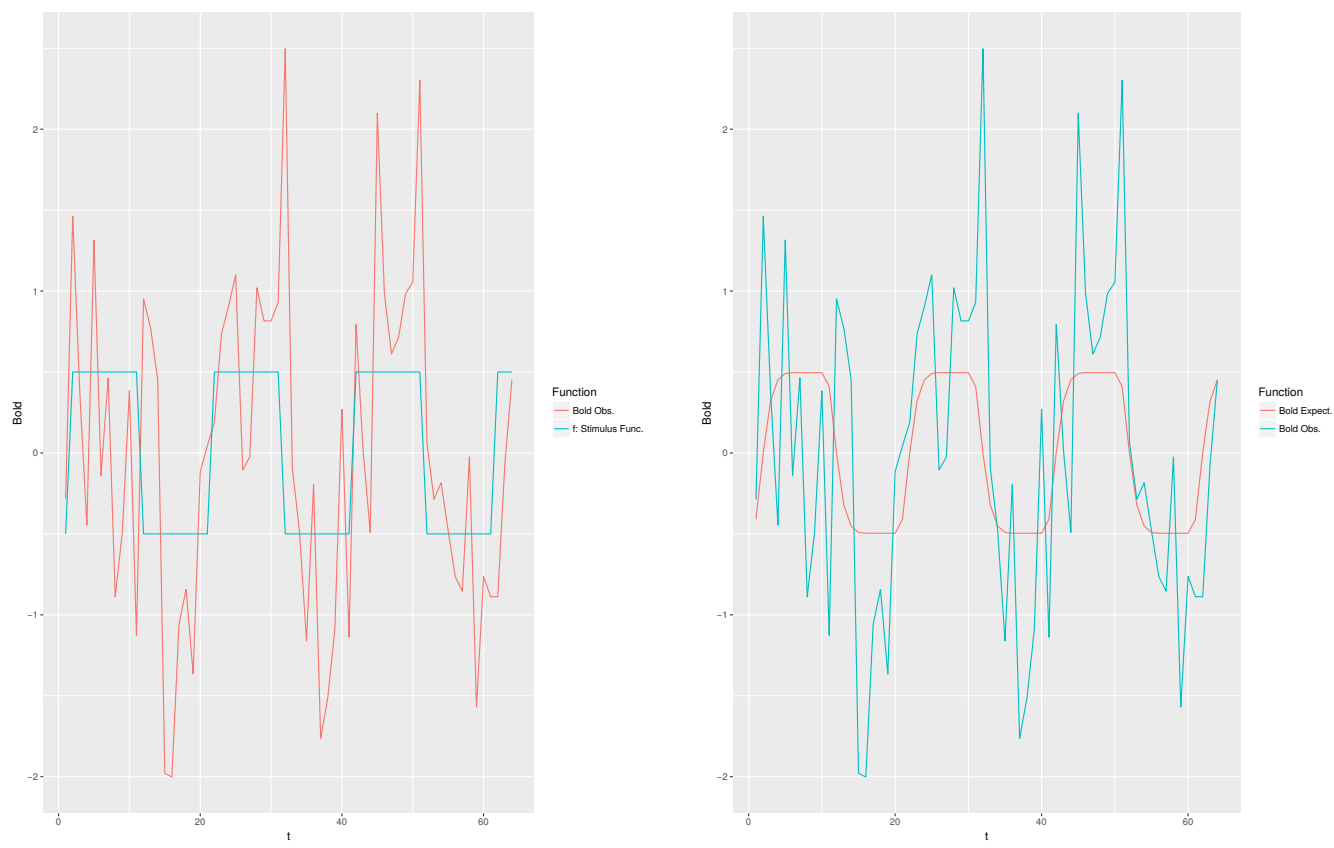

Figure 1.5: Left panel: Observed BOLD response (red line) and the stimulus time series (blue line). Right panel: Observed BOLD response (blue line) and the expected BOLD response (red line).

Choosing an appropriate $\mathrm{HRF}$ is the key to capturing the best shape possible, which will ensure a good fit of the model being used on the BOLD observed when the signal is present ([Poldrack et al., 2011]).

\subsection{Statistical modeling of the BOLD signal}

The most widely-used statistical model among users of the fMRI technique is the so-called General Linear Model (GLM), which is simply a normal linear regression model. There are several reasons that help to explain the popularity and success of this model in the fMRI field, but two very important ones are: its simplicity and robustness; and the fact that it can can be found as a standard tool in the most popular packages for fMRI data analysis. The usual specification of this model is given by

$$
Y_{v}(t)=x(t) \beta_{v}+\epsilon_{v}, \quad \epsilon_{v} \sim N\left(0, \sigma^{2}\right)
$$

for $v=1, \ldots, V$ and $t=1, \ldots, T$, where $V$ is the number of voxels in the fMRI array and $T$ is the number of observations in each time series. $Y_{v}(t)$ and $x(t)$ are the observed (obtained from the scanner) and expected ( obtained from equation 1.1) BOLD response, respectively. Then the parameter $\beta_{v}$ will inform about the time series voxels $\left(Y_{v}\right)$ that match with expected BOLD response $x(t)$. In this type of modeling, a key feature of any fMRI data array is totally ignored: the spatiotemporal relationships. Thus, in the model 1.1, observations are assumed independent inside each voxel (temporal independence), as well as among voxels (spatial independence), both unrealistic suppositions. One of the main consequences of performing this type of analysis is getting incorrect inferences about the $\beta_{v}$ parameter, which could led to have high rates of false positives activations. A common practice to fix this problem is to use some sort of corrections, like the Bonferroni correction or spatial extent methods (Worsley \& Friston [1995]), among others. For instance, in figure 1.6 we can see an example of an activation of the visual cortex. In the left panel, the inference is performed without any type of correction and in the right panel, a Bonferroni correction is used. 
From this example, we might think that this kind of method could help to solve the problem of detecting a false activation pattern. However, Eklund et al. [2012] and Eklund et al. [2016] evaluate the most common software packages for fMRI analysis using real data, and they find that in general, those correction methods do not work very well. Specifically, they use resting-state data and a total of 3 million random task group analyses to compute empirical familywise error rates. For a nominal familywise error rate of $5 \%$, parametric statistical methods are shown to be conservative for voxelwise inference (e.g., using the Bonferroni method) and invalid for clusterwise inference (e.g., using spatial extent methods).

(a)

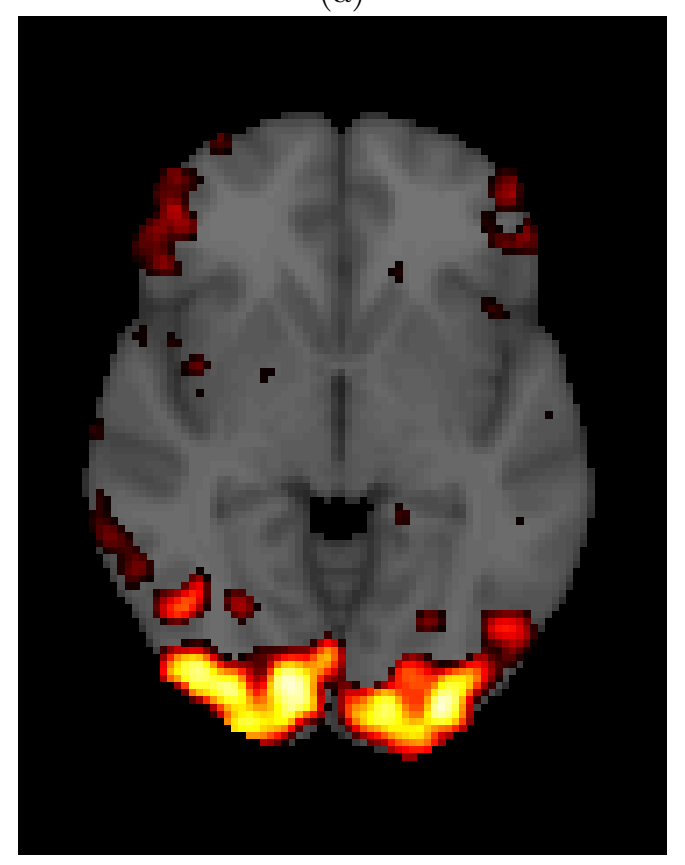

(b)

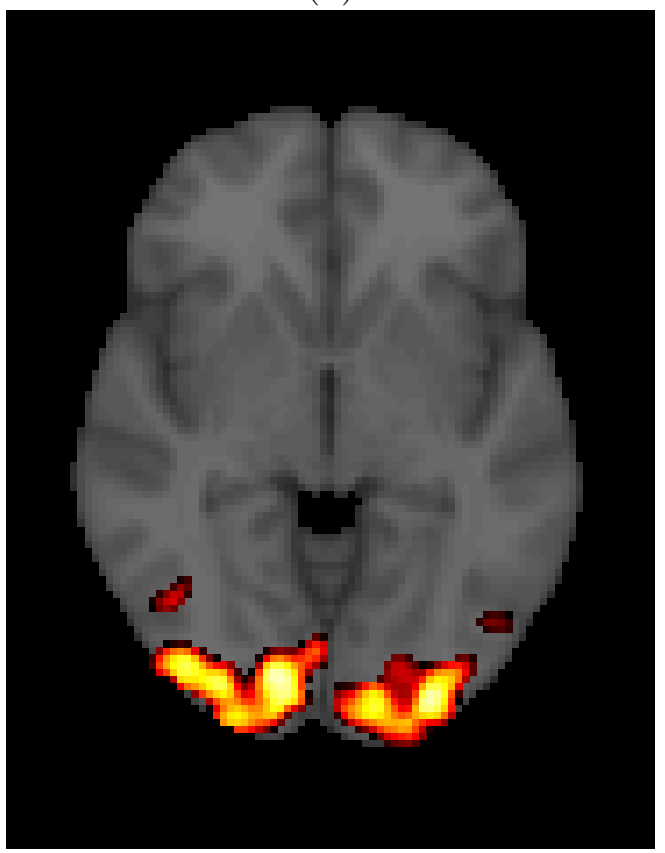

Figure 1.6: (a) Visual cortex activation without Bonferroni correction (a high rate of false activations or detected activations outside the visual cortex). (b) Visual cortex activation with Bonferroni correction (activation detected only in the visual cortex).

\subsubsection{Group analysis}

In fMRI studies, it is very common to perform group experiments, where the main interest could be to study either group activation or compare the activation response between two groups (e.g., patients versus controls). In order to do so, the analysis is divided in two stages. In the first stage, for each individual in each group, a statistical model is used to model the observed BOLD response as is described above. In the second stage, the $\beta_{v i j}$ parameters obtained in the first are used to compute the group effect. Thus, for the $j-t h$ group and the $v-t h$ voxel the average effect is given by

$$
\bar{\beta}_{v j}=\frac{\sum_{i=1}^{n_{j}} \beta_{v i j}}{n_{j}},
$$

where $n_{j}$ is the sample size of the group $j$, for $j=1, . ., m$. For example, when $m=2$ one could perform any of the following tests: $\left\{\bar{\beta}_{v 1}>\bar{\beta}_{v 2}\right\},\left\{\bar{\beta}_{v 2}>\bar{\beta}_{v 1}\right\},\left\{\bar{\beta}_{v j}>0\right\}$ and $\left\{\bar{\beta}_{v j}=0\right\}$. The type of test to be performed depends on the interest of the researcher. 


\subsubsection{About software packages for fMRI analysis}

The most common software packages for fMRI analysis are SPM, FSL and AFNI. Among them, only SPM has a complete option for Bayesian modeling for both first-stage and secondstage analysis. For the first-level analysis, the user can use spatial priors for regression coefficients and regularized voxel-wise $A R(p)$ models for the fMRI noise processes. The second stage uses the empirical Bayes algorithm with global shrinkage priors. However, those stages are not connected. In other words, the second stage does not use the results obtained in the first stage. Thus, if the user wants to perform Bayesian modeling in the second stage, then they must have already estimated a frequentist model in the first stage and used those estimations as an input for the second, which means that with SPM it is not possible to perform fully Bayesian modeling for group fMRI analysis. In the case of FSL there is only a Bayesian option for the second stage or group comparison. In the AFNI package, there are only frequentist modeling options. 


\title{
Assessing Dynamic Effects on a Bayesian Matrix-Variate Dynamic Linear Model: an Application to fMRI data Analysis.
}

\author{
January 19, 2020
}

\begin{abstract}
In this work, we propose a modeling procedure for fMRI data analysis using a Bayesian Matrix-Variate Dynamic Linear Model (MVDLM). With this type of model, less complex than the more traditional temporal-spatial models, we are able to take into account the temporal and -at least locally- the spatial structures that are usually present in this type of data. Despite employing a voxel-wise approach, every voxel in the brain is jointly modeled with its nearest neighbors, which are defined through a euclidian metric. MVDLM's have been widely used in applications where the interest lies in to perform predictions and/or analysis of covariance structures among time series. In this context, our interest is rather to assess the dynamic effects which are related to voxel activation. In order to do so, we develop three algorithms to simulate online-trajectories related to the state parameter and with those curves or simulated trajectories we compute a Monte Carlo evidence for voxel activation. Through two practical examples and two different types of assessments, we show that our method can be viewed for the practitioners as a reliable tool for fMRI data analysis. Despite all the examples and analysis are illustrated just for a single subject analysis, we also describe how more general group analysis can be implemented.
\end{abstract}

Keywords: fMRI, Bayesian Analysis, Matrix-Variate Dynamic Linear Models, Monte Carlo Integration.

\section{Introduction}

Magnetic resonance imaging (MRI) is a non-invasive technique that is used to create elaborate anatomical images of the human body. Specifically, this technique can be used to obtain detailed brain images that can help to identify different types of tissue such as, 
for example, white matter and gray matter, and can also be used to diagnose aneurysms and tumors. Another important facet of this technique is that it can be used to visualize dynamic or functional activity in the brain. Functional Magnetic Resonance Imaging (fMRI) can be described as a generalization of the MRI technique, where the focus is not just one high-resolution image of the brain, but rather a sequence of low-resolution images that allows for identification, at least in an indirect way, of neuronal activity through the blood-oxygen-level dependent (BOLD) contrast (Poldrack et al. [2011]). The sequence of MRI images is obtained using an MRI scanner, essentially a huge magnet, which detects activity in the brain's different regions by tracing blood flows. Statistical models are very useful for analyzing the post-processed data that compose the sequence of images obtained in an fMRI experiment. The most popular statistical model used to identify a brain-region reaction to an external stimulus is the normal regression linear model, usually known as General Linear Model (GLM) in the fMRI literature, which assumes spatiotemporal independence among the data, an unrealistic assumption that can lead to incorrect inference. For instance, Eklund et al. 2016] evaluate the most common software packages (SPM, FSL and AFNI) for fMRI analysis, which have the GLM implemented as one of their analysis tools. Specifically, they evaluate that model for group analysis using real resting-state data and they find that, for a nominal family-wise error rate of $5 \%$, parametric statistical methods are shown to be conservative for voxel-wise inference and invalid for cluster-wise inference. However, there are other alternatives to model fMRI data, specially Bayesian models. Those alternatives usually account for the spatiotemporal structure present in this type of data. For example, Zhang et al. [2016] propose a spatiotemporal Nonparametric Bayesian approach to model both individual and group stages in one-step modeling. For posterior inference, they implement a Markov Chain Monte Carlo (MCMC) algorithm and additionally a suitable variational Bayes algorithm. Eklund et al. [2017] propose a Bayesian heteroscedastic GLM with autoregressive linear noise and heteroscedastic noise innovations for single subject fMRI analysis. They develop an efficient MCMC algorithm that allows for variable selection among the regressors. Bezener et al. 2018] propose a Bayesian Spatiotemporal Modeling for single subject fMRI analysis. Their modeling includes a novel areal model to parcel voxels into clusters and also use an MCMC algorithm for poste- 
rior inference. Yu et al. 2018 propose a Bayesian Variable Selection approach to model Complex-Valued fMRI data. They develop their models applying complex-valued spikeand-slab priors on the parameters associated with brain activation and perform posterior inference via MCMC algorithms. Those methods are just some of the most recent works published in the field. See Zhang et al. 2015] and Bowman [2014 for a more complete review of statistical methods for fMRI data analysis.

In this work, we propose a local spatiotemporal Bayesian modeling for two-stage fMRI data analysis. However, here we do not resort to the traditional spatiotemporal models that are usually used to model fMRI data in the Bayesian contex. Instead, we use a Matrix-Variate Dynamic Linear Model (MDLM) (see West and Harrison, 1997, p. 581] for more details about these types of models), specifically, we employ the model proposed by Quintana [1985] and Quintana [1987] to model the BOLD response related to block and event-related experiments ( see Kashou 2014 for a detailed explanation of these kinds of designs). With this type of modeling, we can easily account for the temporal dependence through the latent component of the model which is most commonly known as the state equation. Despite the fact that, we take a voxel-wise approach, we model every voxel jointly with their nearest neighbors and thus the resulting covariance structure is used to perform the voxel-wise inference related to brain activation. This is what we call local spatiotemporal modeling. To define the clusters or neighborhoods related to every voxel, we just employ an Euclidean distance. Thus, the modeler has to define a fixed distance $r$ and all the voxels lying within that distance will be considered part of the cluster. Before explaining how this modeling is used to detect brain activation, it is worth mentioning that most of the work related to MDLM has been devoted to forecasting and analyzing covariance structure across several time series (Quintana and West [1987], Aguilar and West [2000], Fei et al. [2011]), however not much work has been developed regarding the inference of effect sizes related to regressors in an MDLM. West and Harrison, 1997, p. 280] briefly mention how to perform inference about the dynamic regression coefficient or state parameter, but only for a particular time $t$ within the interval of observed time. In the case of an fMRI modeling, we would like to perform inference on the state parameter for an entire interval of time at 
once. In that sense, we propose three algorithms to draw on-line estimated trajectories of the state parameter and thus compute a measure of evidence via Monte Carlo integration. Hence, with that measure of evidence, we can perform inference about the state parameter or equivalently, in the context of fMRI modeling, about brain reaction. One algorithm is just an implementation of a forward-filtering-backward-sampling algorithm which was suggested by Frühwirth-Schnatter [1994 to draw state parameters for univariate dynamic linear models. In this matrix-variate setting, we use the same idea, but instead of estimating individual parameters for the covariance matrix of the state parameter in the Evolution equation (1) using data augmentation, we use discount factors to deal with those quantities (Ameen and Harrison [1985]). The remain two algorithms can be described as forwardsampling schemes, in the sense that here we do not resort to the filtering distributions and just use the posterior distributions computed at each observed time $t$.

Thus, at the individual stage, an MDLM is fitted for every subject in the sample, and analysis such as brain activation and/or contrast between tasks can be performed using any of the three algorithms mentioned above. At the group stage, the individual posterior distributions for the state parameters are combined in a suitable way, obtaining new distributions that represent the updated belief for the group effect. In this way, all of the analysis usually performed in a fMRI experiment (brain activation, task comparison, and group comparison) can be performed again by using any of the three algorithms proposed in this work. In order to show the performance of our method, we present two real data examples related to visual and motor task experiments respectively. We also assess our method in two ways: 1) following the same approach as in Eklund et al. [2016] using resting-state fMRI data from healthy controls, obtained from the 1000 Functional Connectomes Project Biswal et al. [2010]. Here we define a fictitious stimulus and seek for the rate of false positives given the null hypothesis of no brain reaction. 2) Using simulated data obtained from the $\mathrm{R}$ package neuRosim [Welvaert et al., 2011].

In the next section, we give a brief description of the Matrix-Variate Dynamic Linear Model and its posterior distribution under a matrix Normal/inverse Wishart prior distribution. We also describe the modeling of the BOLD response using this type model for single and group analysis. Section 3 describes the three algorithms and computation of Monte Carlo 
evidence for voxel activation. In section 4 two examples for block and event-related designs are presented to illustrate the performance of our proposal. In section 5 an assessment is performed to evaluate the capacity of our method to deal with false-positive activations. In the final section, we present some concluding remarks and potential open problems for future development.

\section{$2 \quad$ MVDLM and fRMI Modeling}

The general theory of the MVDLM is presented in Quintana 1985, Quintana 1987 and West and Harrison [1997]. Despite this model having been conceived for forecasting, in this work it is used for a different purpose: model fMRI data related to block and event-related designs through a regression structure in order to identify brain reaction. In other words, we focus our interest on the estimation of, and inference on, the state parameter. Thus, suppose that we have a $q \times 1$ vector $\mathbf{Y}_{t}$, which can be modeled in terms of observation and evolution or state equations as follows.

$$
\begin{array}{ll}
\text { Observation: } & \mathbf{Y}_{t}^{\prime}=\mathbf{F}_{t}^{\prime} \boldsymbol{\Theta}_{t}+\boldsymbol{\nu}_{t}^{\prime} \\
\text { Evolution: } & \boldsymbol{\Theta}_{t}=\mathbf{G}_{t} \boldsymbol{\Theta}_{t-1}+\boldsymbol{\Omega}_{t},
\end{array}
$$

where, for each $t$ we have a $q \times 1$ vector $\boldsymbol{\nu}_{t}$ of observational errors, a $p \times q$ matrix $\boldsymbol{\Theta}_{t}$ of state parameters, a $p \times q$ matrix $\boldsymbol{\Omega}_{t}$ of evolution errors. The $1 \times p$ and $p \times p$ matrices $\mathbf{F}_{t}^{\prime}$ and $\mathbf{G}_{t}$ respectivelly are common to each of the $q$ univariate DLMs. The covariates related to the design being used, either a block or an event-related design as well as other characteristics of the subjects, can be included in the columns of $\mathbf{F}_{t}^{\prime}$. It is supposed that $\nu_{t} \sim N_{q}\left[\mathbf{0}, V_{t} \boldsymbol{\Sigma}\right]$, independently over time, where $\boldsymbol{\Sigma}$ defines the cross-sectional covariance structure for the multivariate model and $V_{t}$ is a known observational scale factor. For the random matrix $\Omega_{t}$ the distribution is given by $\Omega_{t} \sim N_{p q}\left[\mathbf{0}, \mathbf{W}_{t}, \boldsymbol{\Sigma}\right]$, a matrix-variate normal distribution with mean matrix $\mathbf{0}$, left $p \times p$ variance matrix $\mathbf{W}_{t}$ and right variance matrix $\boldsymbol{\Sigma}$ (Dawid 1981]). It is also supposed the initial prior for $\boldsymbol{\Theta}_{0}$ and $\boldsymbol{\Sigma}$ is matrix normal/inverse Wishart, namely $\left(\boldsymbol{\Theta}_{0}, \boldsymbol{\Sigma} \mid D_{0}\right) \sim N W_{n_{0}}^{-1}\left[\mathbf{m}_{0}, \mathbf{C}_{0}, \mathbf{S}_{0}\right]$, for some known defining parameters $\mathbf{m}_{0}, \mathbf{C}_{0}, \mathbf{S}_{0}$ and $n_{0}$ ([Quintana, 1987] [Chapter 3]). $D_{t}=\left\{\mathbf{Y}_{0}, \mathbf{Y}_{1}, \ldots, \mathbf{Y}_{t}\right\}$ are the data observed at time $t$. 


\subsection{Voxel-wise Individual Analysis}

One of the main objectives in an fMRI experiment is to identify a brain reaction in response to some controlled external stimuli, in other words, to look for changes in the BOLD signal related to some experimental manipulation. In order to do that, one can use a block design, an event-related design or even possible, a new design from the composition of those two (Kashou [2014]). The modeling we propose here relies on something called the expected BOLD response, which is obtained as the convolution of the stimulus time series $f$, which is obtained from the design being used, and the hemodynamic response function $h$ (HRF), which can be interpreted as the picture over time of the change of the BOLD signal. In the examples and analysis presented here we use the canonical HRF, which is defined as the difference (between) of two gamma densities. However, it is worth mentioning that any other form from the available options for the HRF can be used. This subject is discussed in depth in Poldrack et al. [2011] and Penny et al. [2011]. In general, the expected BOLD response can be obtained as follows:

$$
x(t)=(h * f)(t)=\int h(\tau) f(t-\tau) d \tau .
$$

Thus, the aim is to identify the fMRI time series that matches the expected BOLD response. For that purpose, we model the observed BOLD response as a linear function of the expected BOLD response using the model (1).

\subsubsection{Individual Modeling}

Let $y_{[i, j, k], t, 1}^{*(z)}$ and $x^{(z)}(t)$ be the observed and expected BOLD response, respectively at brain position $\{i, j, k\}$, time $t$ and subject $z$, for $i=1, \ldots, d_{1}, j=1, \ldots, d_{2}, k=1, \ldots, d_{3}$, $t=1, \ldots, T$ and $z=1 \ldots, N_{g}$. Something that can be noticed from this last definition is that $x^{(z)}(t)$ is supposed to be the same for all the locations in the brain image, which means, it is supposing that the BOLD response is the same in all brain regions. This may be an unrealistic assumption, but it is one that works well in practice. Let the vector 


$$
\mathbf{Y}_{[i, j, k] t}^{(z)}=\left(\begin{array}{c}
y_{[i, j, k], t, 1}^{*(z)} \\
y_{[i+1, j, k], t, 2}^{(z)} \\
y_{[i-1, j, k], t, 3}^{(z)} \\
y_{[i, j+1, k], t, 4}^{(z)} \\
y_{[i, j-1, k], t, 5}^{(z)} \\
y_{[i, j, k+1], t, 6}^{(z)} \\
y_{[i, j, k-1], t, 7}^{(z)}
\end{array}\right)_{1 \times q}
$$

represent the cluster or neighborhood of size $q=7$ of the voxel at position $\{i, j, k\}$ and time t. Thus, we model $\mathbf{Y}_{[i, j, k] t}^{(z)}$ using the model $(1)$, with $\mathbf{G}_{t}=\mathbf{I}_{p}$ and $\mathbf{F}_{t}^{\prime(z)}=\left(x_{1}^{(z)}(t), \ldots, x_{p}^{(z)}(t)\right)$, where $x_{1}^{(z)}(t), \ldots, x_{p}^{(z)}(t)$ are related to different types of stimuli or tasks performed in the fMRI experiment. What we intend with this type of modeling is to capture the covariance structure within the cluster of voxels through the matrix $\Sigma^{(z)}$. The criterion to define the cluster form is based on the Euclidean distance, where the distance between the voxel $V^{*}$ and the neighboring voxel $V_{\sim}$ is given by $d\left(V^{*}, V_{\sim}\right) \leq r$. In figure 1 (right panel), we can see a graphical illustration of a neighborhood of voxels related to the observed BOLD response vector (3) and its corresponded matrix parameter on equation (4), where $r=1$ and consequently $q=7$. In the R-packag $\AA^{1}$ where this method has been implemented, a range of different $r$ values can be set by the users: $r=1,2,3,4$, which consequently leads to $q=7,19,27,33$.

\footnotetext{
${ }^{1}$ https://github.com/JohnatanLAB/BayesDLMfMRI
} 

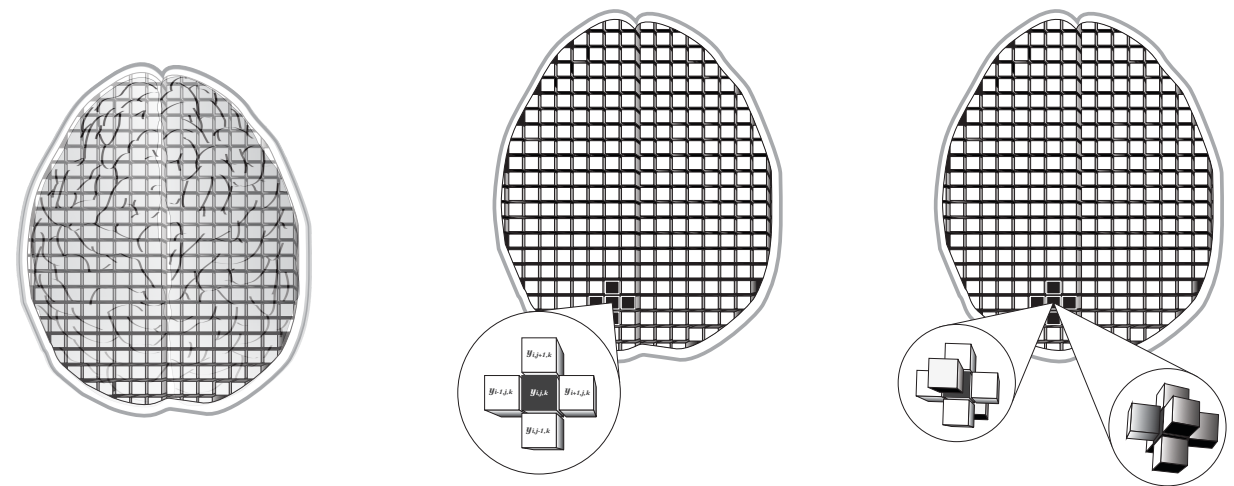

Figure 1: Graphical illustration of a neighborhood of voxels, for $r=1$.

$$
\boldsymbol{\Theta}_{[i, j, k] t}^{(z)}=\left(\begin{array}{ccccccc}
\theta_{[i, j, k], t, t, 1}^{*(z)} & \theta_{[i, j, k], t, 1,2}^{(z)} & \theta_{[i, j, k], t, 1,3}^{(z)} & \theta_{[i, j, k], t, 1,4}^{(z)} & \theta_{[i, j, k], t, 1,5}^{(z)} & \theta_{[i, j, k], t, 1,6}^{(z)} & \theta_{[i, j, k], t, 1,7}^{(z)} \\
\vdots & \vdots & \vdots & \vdots & \vdots & \vdots & \vdots \\
\theta_{[i, j, k], t, p, 1}^{*(z)} & \theta_{[i, j, k], t, p, 2}^{(z)} & \theta_{[i, j, k], t, p, 3}^{(z)} & \theta_{[i, j, k], t, p, 4}^{(z)} & \theta_{[i, j, k], t, p, 5}^{(z)} & \theta_{[i, j, k], t, p, 6}^{(z)} & \theta_{[i, j, k], t, p, 7}^{(z)}
\end{array}\right) .
$$

From here to the rest of the article, we change the notation related to the location subscript $[i, j, k]$ by $v$.

\section{Posterior inference}

Under the assumptions and prior distributions defined above and following the results in Dawid [1981], it can be shown that the joint posterior distribution of the state parameter $\boldsymbol{\Theta}_{v t}^{(z)}$ and the covariance matrix $\boldsymbol{\Sigma}_{v}^{(z)}$ is given by

$$
\left(\mathbf{\Theta}_{v t}^{(z)}, \boldsymbol{\Sigma}_{v}^{(z)} \mid D_{v t}\right) \sim N W_{n_{t}}^{-1}\left[\mathbf{m}_{v t}^{(z)}, \mathbf{C}_{v t}^{(z)}, \mathbf{S}_{v t}^{(z)}\right]
$$

with

$$
\begin{gathered}
\mathbf{m}_{v t}^{(z)}=\mathbf{m}_{v, t-1}^{(z)}+\mathbf{A}_{v t}^{(z)} \mathbf{e}_{v t}^{\prime(z)} \text { and } \mathbf{C}_{v t}^{(z)}=\mathbf{C}_{v, t-1}^{(z)}+\mathbf{W}_{v t}^{(z)}-\mathbf{A}_{v t}^{(z)} \mathbf{A}_{v t}^{\prime(z)} Q_{v t}^{(z)}, \\
n_{t}=n_{t}+1 \text { and } \mathbf{S}_{v t}^{(z)}=n_{t}^{-1}\left[n_{t-1} \mathbf{S}_{v, t-1}^{(z)}+\mathbf{e}_{v t}^{(z)} \mathbf{e}_{v t}^{(z)} / Q_{v t}^{(z)}\right]
\end{gathered}
$$

where 
$Q_{v t}^{(z)}=V_{t}+\mathbf{F}_{t}^{\prime(z)}\left[\mathbf{C}_{v, t-1}^{(z)}+\mathbf{W}_{v t}^{(z)}\right] \mathbf{F}_{t}^{(z)}, \quad \mathbf{A}_{v t}^{(z)}=\left[\mathbf{C}_{v, t-1}^{(z)}+\mathbf{W}_{v t}^{(z)}\right] F_{t}^{(z)} / Q_{v t}^{(z)}, \quad$ and $\mathbf{e}_{v t}^{(z)}=\mathbf{Y}_{v t}^{(z)}-\mathbf{f}_{v t}^{(z)}$

$\mathbf{f}_{v t}^{(z)}$ is the mean of the predictive distribution $p\left(\mathbf{Y}_{v t}^{(z)} \mid D_{v, t-1}^{(z)}\right)$. Hence, the marginal posterior distribution for the state parameter is given by

$$
\left(\mathbf{\Theta}_{v t}^{(z)} \mid D_{v t}^{(z)}\right) \sim T_{n_{t}}\left[\mathbf{m}_{v t}^{(z)}, \mathbf{C}_{v t}^{(z)}, \mathbf{S}_{v t}^{(z)}\right]
$$

which is known as a matrix $T$ distribution with $p \times q$ mean matrix $\mathbf{m}_{v t}^{(z)}, p \times p$ left variance matrix $\mathbf{C}_{v t}^{(z)}$, and $q \times q$ right variance matrix $\mathbf{S}_{v t}^{(z)}$. To deal with the unknown matrix parameter $\mathbf{W}_{v t}^{(z)}$, we adopt the discount model approach proposed by Ameen and Harrison 1985 in the same manner as in West et al. 1985. Thus, we express the system variace matrix as $\mathbf{W}_{v t}^{(z)}=\mathbf{B}_{\mathbf{t}} \mathbf{C}_{v, t-1}^{(z)} \mathbf{B}_{\mathbf{t}}-\mathbf{C}_{v, t-1}^{(z)}$, where $\mathbf{B}_{\mathbf{t}}$ is an $p \times p$ diagonal matrix of positive discount factors $1 / \beta_{i t}^{1 / 2}$, for $i=1, \ldots, p$ and $0<\beta_{i t} \leq 1$. As we show in one of the examples below, those discount factors are a key component of this modeling in the sense that they can affect the sensibility of detection of brain activation.

A reasonable approximation for the posterior distribution (6) when $n_{t} \geq 30$ is given by

$$
\left(\Theta_{v t}^{(z)} \mid D_{v t}^{(z)}\right) \stackrel{a p p r o x}{\sim} N_{p q}\left[\mathbf{m}_{v t}^{(z)}, \mathbf{C}_{v t}^{(z)}, \mathbf{S}_{v t}^{(z)}\right] .
$$

We have two main considerations to justify working only with posterior distributions for $n_{t} \geq 30$. The first is that as we use vague prior distributions at $t=0$, the sequential update process takes some period of time before reaching a posterior distribution dominated by the data. Then, the first posterior distributions (i.e., for $t<30$ ) could be considered irrelevant for the analysis. The second consideration is simply that dealing with normal distributions simplifies the mathematical work when dealing with linear combinations, which are quite common in this work when inference is performed on the matrix parameter $\boldsymbol{\Theta}_{v t}^{(z)}$ to detect brain activation.

As mentioned above, a brain activation related to the stimulus or task $l$ (for $l=1, \ldots, p$ ) is identified when the expected BOLD response $\left(x_{l}^{(z)}(t)\right)$ matches the observed BOLD response $\left(\mathbf{Y}_{v t}^{(z)}\right)$. In other words, a brain activation in the cluster of voxels $v$ related to the $l$-th task or stimulus is equivalent to the $l$-th row of $\boldsymbol{\Theta}_{v t}^{(z)}$ being positive. In this sense, we define 
three different ways to evaluate brain activation, which are related to the following variables obtained from the components of $\boldsymbol{\Theta}_{v t}^{(z)}$

$$
\text { Marginal effect: } \theta_{v t, l}^{*(z)}=\theta_{v, t, l, 1}^{*(z)}
$$

$$
\text { Average cluster effect: } \bar{\theta}_{v t, l}^{(z)}=\frac{1}{q}\left[\theta_{v, t, l, 1}^{*(z)}+\sum_{n=2}^{q} \theta_{v, t, l, n}^{(z)}\right]
$$

$$
\text { Joint effect: } \boldsymbol{\theta}_{v t, l}^{(z)}=\left(\theta_{v, t, l, 1}^{*(z)}, \theta_{v, t, l, 2}^{(z)}, \ldots, \theta_{v, t, l, q}^{(z)}\right) .
$$

The marginal effect (8) is when just a marginal distribution from (7) is considered, in other words when the correlation captured by $\mathbf{S}_{v t}^{(z)}$ is ignored. It can be shown that under particular considerations a DLM is equivalent to a traditional static regression linear model (or GLM in the fMRI context), so an analysis using marginal effects can yield results pretty similar to those obtained when performing a voxel-wise approach using a GLM. Nevertheless, this depends on the variance value of the state parameter $\left(\mathbf{W}_{v t}^{(z)}=0\right)$. The average (9) and joint (10) effects are more interesting in the sense that they take advantage of the information contained within the entire cluster of voxels.

From the properties of the matrix normal distribution, the distributions of (8), (9) and (10) are given by

$$
\begin{gathered}
\theta_{v t, l}^{*(z)} \mid D_{v t}^{*(z)} \sim N\left(m_{v t, l, 1}^{*(z)}, C_{v t, l, l}^{(z)} S_{v t, 1,1}^{(z)}\right), \\
\bar{\theta}_{v t, l}^{(z)} \mid D_{v t}^{(z)} \sim N\left(\bar{m}_{v t, l}^{(z)}, \bar{S}_{v t, l}^{(z)}\right), \\
\boldsymbol{\theta}_{v t, l}^{(z)} \mid D_{v t}^{(z)} \sim N_{q}\left(\boldsymbol{m}_{v t, l}^{(z)}, C_{v t, l, l}^{(z)} \boldsymbol{S}_{v t}^{(z)}\right),
\end{gathered}
$$

where $C_{v t, l, l}^{(z)}$ and $S_{v t, n, n}^{(z)}$ are the elements on the main diagonal of the matrices $\boldsymbol{C}_{v t}^{(z)}$ and $\boldsymbol{S}_{v t}^{(z)}$ respectively, $\bar{m}_{v t, l}=\frac{1}{q} \sum_{n=1}^{q} m_{v t, l, n}, \bar{S}_{v t, l}=\frac{1}{q^{2}}\left[\sum_{n=1}^{q} C_{v t, l, l} S_{v t, n, n}+\sum_{n \neq n^{\prime}} C_{v t, l, l} S_{v t, n, n^{\prime}}\right]$ and $D_{v t}^{*(z)}$ is the data related to first component of the vector (3). With the distributions (11), (12) and (13), at least from our knowledge, it is only possible to perform inference (about (8), (9) 
and (10) for a particular fixed time $t$, which in the context of fMRI means inference related to only one brain image. However, in fMRI data analysis, it is more relevant to perform an inference procedure at once for the entire group of brain images just to understand brain dynamics related to the controlled stimulation presented on the fMRI experiment. In the next section, we present three algorithms that allow us to draw dynamic trajectories of the state parameter related to any of (8), (9) and (10) and hence using those trajectories to compute a measure of evidence for voxel activation on the entire observed interval of brain images. But before we get there, we first offer a brief explanation on how to get versions of (8), (9) and (10) for the group stage analysis.

\subsection{Voxel-wise Group Analysis}

Now, we describe the fMRI group analysis where the focus is to detect an average group activation (single-group analysis) and/or compare voxel activation between two groups (e.g. patients vs. controls). Here we take any of the posterior distributions (11), (12), or (13), depending on the case, as an input for this stage. For instance, let us suppose that a sample of $N_{g}$ subjects are part of an fMRI experiment where $p$ stimuli are presented. Thus, taking advantage of the properties of normal distriution, we obtain the following distributions for the group stage:

$$
\begin{gathered}
\text { Marginal group effect: } \bar{\theta}_{v t, l}^{*(g)} \sim N\left(\bar{m}_{v t, l}^{*(g)}, \bar{S}_{v t, l}^{(g)}\right), \\
\text { Average cluster group effect: } \overline{\bar{\theta}}_{v t, l}^{(g)} \sim N\left(\overline{\bar{m}}_{v t, l}^{(g)}, \overline{\bar{S}}_{t, l}^{(g)}\right), \\
\text { Joint group effect: } \overline{\boldsymbol{\theta}}_{v t, l}^{(g)} \sim N_{q}\left(\overline{\boldsymbol{m}}_{v t, l}^{(g)}, \overline{\boldsymbol{S}}_{t}^{(g)}\right),
\end{gathered}
$$

where, 


$$
\begin{aligned}
& \bar{m}_{v t, l}^{*(g)}=\frac{1}{N_{g}} \sum_{z=1}^{N_{g}} m_{v t, l, 1}^{*(z)} \quad \bar{S}_{v t, l}^{(g)}=\frac{1}{N_{g}^{2}} \sum_{z=1}^{n_{g}} C_{v t, l, l}^{(z)} * S_{v t, 1,1}^{(z)}, \\
& \overline{\bar{m}}_{v t, l}^{(g)}=\frac{1}{N_{g}} \sum_{z=1}^{n_{g}} \bar{m}_{v t, l}^{(z)} \quad \overline{\bar{S}}_{v t, l}^{(g)}=\frac{1}{N_{g}^{2}} \sum_{z=1}^{N_{g}} \bar{S}_{v t, l}^{(z)}, \\
& \overline{\boldsymbol{m}}_{v t, l}^{(g)}=\frac{1}{N_{g}} \sum_{z=1}^{N_{g}} \boldsymbol{m}_{v t, l}^{(z)} \quad \overline{\boldsymbol{S}}_{v t}^{(g)}=\frac{1}{N_{g}^{2}} \sum_{z=1}^{n_{g}} C_{v t, l, l}^{(z)} \boldsymbol{S}_{v t}^{(z)} .
\end{aligned}
$$

Let us suppose now there are two groups we want to compare. For the patiens' group (group $\boldsymbol{A}$ ), we have $N_{A}$ individuals and for the controls' group (group $\boldsymbol{B}$ ), we have $N_{B}$ individuals. Thus, for instance, if we decide to compare the joint effect between the two groups, the most obvious way to do so is by computing the distribution for the variable $\overline{\boldsymbol{\theta}}_{v t, l}^{(A B)}=\overline{\boldsymbol{\theta}}_{v t, l}^{(A)}-\overline{\boldsymbol{\theta}}_{v t, l}^{(B)}$, which in this case is given by $N_{q}\left(\overline{\boldsymbol{m}}_{v t, l}^{(A)}-\overline{\boldsymbol{m}}_{v t, l}^{(B)}, \overline{\boldsymbol{S}}_{t}^{(A)}+\overline{\boldsymbol{S}}_{t}^{(B)}\right)$. The analysis is analogous for the marginal and average effects. As it was mentioned above, under this setting, it is only possible to perform any type of inference for a particular fixed time $t$. In the next section, we present three algorithms that help us to overcome this limitation.

\section{Algorithms}

In this section we present three different algorithms to draw on-line trajectories of the estate parameter. The first two algorithms only depends on each posterior distribution obtained at each time $t$, whereas the third one is based on the filtered distribution $p\left(\boldsymbol{\Theta}_{v, t-j}^{(z)}, \boldsymbol{\Sigma}^{(z)} \mid D_{v t}^{(z)}\right)$, for $1<j \leq t$. For the examples and assessment presented on sections (4) and (5), we implement model (1) along these algorithms in an $\mathrm{R}$ package $\AA^{2}(\mathrm{R}$ Core Team [2018|) with the help of the RcppArmadillo (Eddelbuettel and Sanderson [2014]) and parallel packages to speed up the computation time. We also make use of the oro.nifti Whitcher et al., 2011 and neurobase Muschelli, 2018 packages to import and export the fMRI data on nii.gz format and plot the evidence activation maps. The FLS software (Jenkinson et al. 2012]) is used when preprocessing of the fMRI data is needed.

\footnotetext{
${ }^{2}$ https://github.com/JohnatanLAB/BayesDLMfMRI
} 


\subsection{Forward Estimated Trajectories Sampler or FEST algoritm}

Let us suppose we are interested in detect brain activation using the distribution (12). Then, we define the online estimated trajectory of $\boldsymbol{\theta}_{v t, l}^{(z)}$ as the vector:

$$
\gamma_{v, l}^{(z)}=\left\{E\left(\boldsymbol{\theta}_{v t_{1}, l}^{(z)} \mid D_{v t_{1}}^{(z)}\right), E\left(\boldsymbol{\theta}_{v t_{2}, l}^{(z)} \mid D_{v t_{2}}^{(z)}\right), \ldots, E\left(\boldsymbol{\theta}_{v T, l}^{(z)} \mid D_{v T}^{(z)}\right)\right\},
$$

for $t_{1} \geq 30$. In case of a significant match between the expected and observed BOLD responses, one would expect every component of the vector $\gamma_{v, l}^{(z)}$ being positive. In this sense, we must compute a measure of evidence for brain activation at voxel $v$, such as $p\left(\gamma_{v, l}^{(z)}>0\right)$. For instance, if $p\left(\gamma_{v, l}^{(z)}>0\right)>\alpha$, where $\alpha$ is a threshold defined by the user (e.g. $\alpha=0.95$ ), then one would conclude that there is significant evidence of a match between the expected and the observed BOLD responses. In other words, there is a brain activation related to the $l$-th stimulus or task in the cluster related to the voxel $v$. The functional variable $\gamma_{v, l}^{(z)}$ can be defined in the same way in case of using either 111 or (13). Thus, taking advantage of the sequential update process related to the posterior distribution (7), we build an algorithm to draw $\gamma_{v, l}^{(z)}$ variables. We name it as Forward Estimated Trajectories Sampler (FETS).

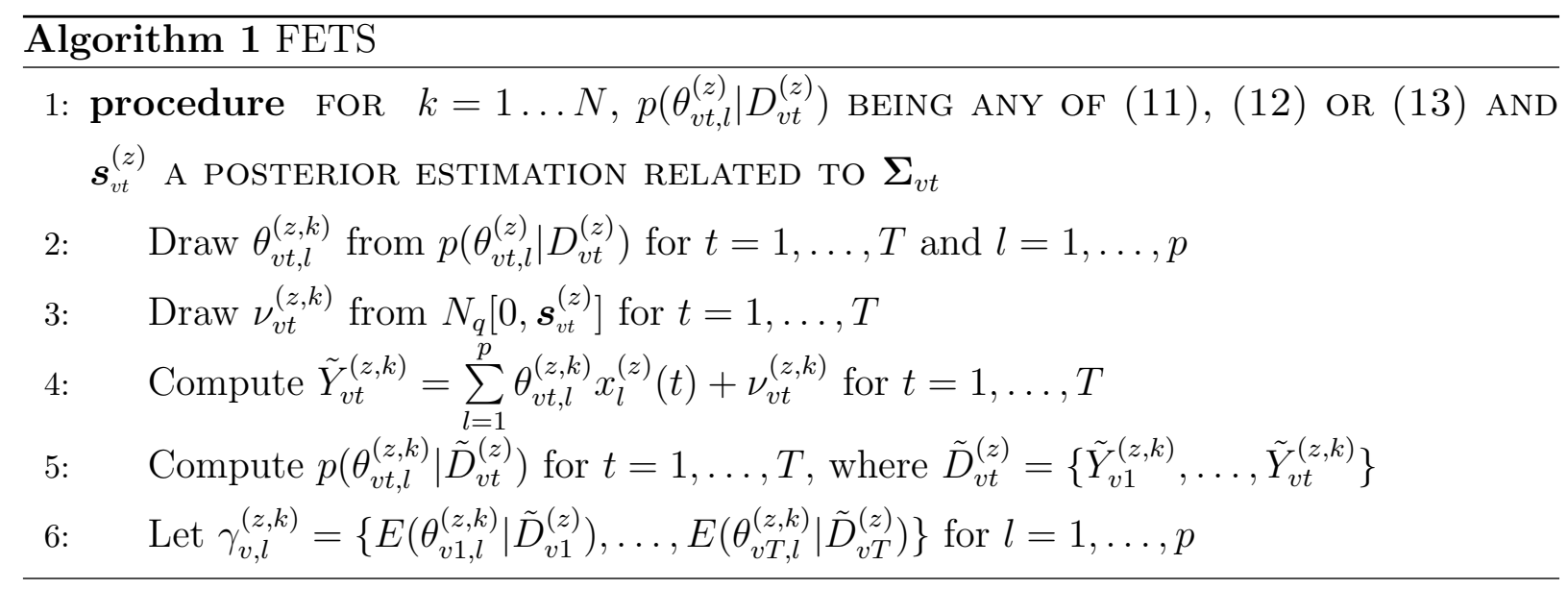

Then, a measure of evidence for the activation of voxel $v$ is computed as

$$
p\left(\gamma_{v, l}^{(z)}>0\right)=E\left(\mathbb{1}_{\left(\gamma_{v, l}^{(z)}>0\right)}\right) \approx \frac{\sum_{k=1}^{N} \mathbb{1}_{\left(\gamma_{v, l}^{(z, k)}>0\right)}}{N},
$$

where $\mathbb{1}_{(A)}$ is the indicator function related to the event $A$. The estimation $\boldsymbol{s}_{v t}^{(z)}$ depends on the chioce for $p\left(\theta_{v t, l}^{(z)} \mid D_{v t}^{(z)}\right)$. Thus, for any of 11, , 12 or $13,, \boldsymbol{s}_{v t}^{(z)}$ can be replaced 
by $S_{v t, 1,1}^{(z)}, \frac{1}{q^{2}}\left[\sum_{n=1}^{q} S_{v t, n, n}^{(z)}+\sum_{n \neq n^{\prime}} S_{v t, n, n^{\prime}}^{(z)}\right]$ or $\mathbf{S}_{v t}^{(z)}$ respectively. It is also possible to compute a measure of evidence for a contrast between two different tasks or stimuli. For instance, let's suppose one is interested in the comparison $\gamma_{v, l}^{(z)}>\gamma_{v, l^{\prime}}^{(z)}$. In other words, he or she wants to investigate whether the brain activation related to the task $l$ is stronger than that related to task $l^{\prime}$. To answer that question, one can compute $p\left(\gamma_{v, l}^{(z)}-\gamma_{v, l^{\prime}}^{(z)}>0\right)$ taking the algorithm's output in the step 6 and using Mote Carlo integration analogously as in (17). Besides of the use of the FEST algorithm to compute measures of evidence for brain activation, it also generates other useful outputs such as $\left\{\tilde{Y}_{v 1}^{(z, k)}, \ldots, \tilde{Y}_{v t}^{(z, k)}\right\}$ and $\left\{\mathbf{S}_{v 1}^{(z, k)}, \ldots, \mathbf{S}_{v t}^{(z, k)}\right\}$, which could eventually be used to build model assessment methods and/or to approach the conectivity problem between different brain regions. These issues are discussed in more detail in the Future Work section. The use of the FEST algorithm for the group case is straightforward and it just requiers the use of any of the distributions in (14), (15) or 16 playing the role of $p\left(\theta_{v t, l}^{(z)} \mid D_{v t}^{(z)}\right)$ and an appropriate estimation for observational noise variance $\boldsymbol{\Sigma}_{v t}^{(z)}$, which in this case can be computed as $\overline{\boldsymbol{S}}_{v t}^{(g)}=\frac{1}{N_{g}^{2}} \sum_{z=1}^{n_{g}} \boldsymbol{S}_{v t}^{(z)}$. However, it requieres that the covariates related to $\mathbf{F}_{v t}^{\prime}(z)$ must be the same for all the subjects. This last is a common feature in many fMRI experiments as we will see in one of the examples presented below. However, some designs such as event-related designs occasionally requiere random sequences of stimuli, which lead to have different $\mathbf{F}_{v t}^{\prime(z)}$ matrices for each subject in the sample. To overcome this limitation, we propose a second and simpler algorithm, which does not depend on $\mathbf{F}_{v t}^{\prime(z)}$ and can also be used to perform inference about the state parameter.

\subsection{FSTS algorithm}

The evolution equation in (1) relates to the state parameter at time $t$ with its own value at time $t-1$. We take advantage of that Markovian property, some prior-posterior estimations and the concept of discount factors to develop a forward sampler algorithm, which we called Forward State Trajectories Sampler (FSTS).

From the vector $\hat{\boldsymbol{\Theta}}_{v}^{(z, k)}=\left\{\boldsymbol{\Theta}_{v 1}^{(z, k)}, \ldots, \boldsymbol{\Theta}_{v T}^{(z, k)}\right\}$, we can define three different types of subvectors related to the variables in (8), (9) and (10). For instance, if one is interested in the 


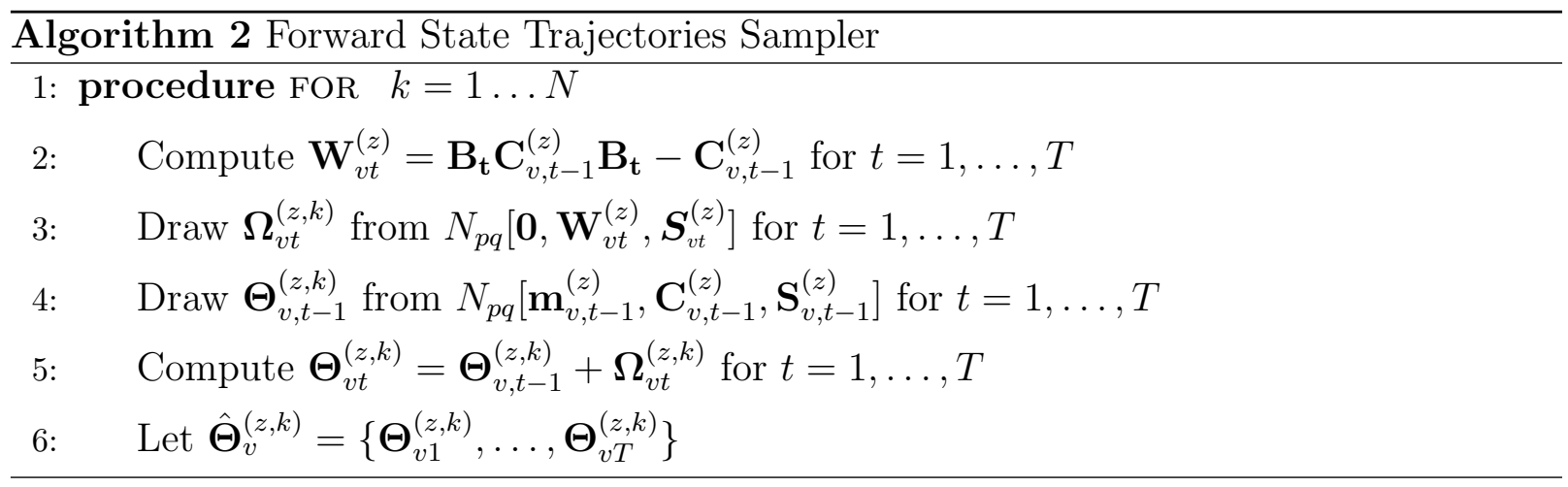

joint effect $\sqrt{10}$, then $\hat{\boldsymbol{\theta}}_{v l}^{(z)}=\left\{\boldsymbol{\theta}_{v 1, l}^{(z)}, \ldots, \boldsymbol{\theta}_{v T, l}^{(z)}\right\}$, for $l=1, \ldots, p$. Thus, for the task $l$ one can compute an activation probability for the voxel $v$ as

$$
p\left(\hat{\boldsymbol{\theta}}_{v l}^{(z)}>0\right)=E\left(\mathbb{1}_{\left(\hat{\boldsymbol{\theta}}_{v l}^{(z)}>0\right)}\right) \approx \frac{\sum_{k=1}^{N} \mathbb{1}_{\left(\hat{\boldsymbol{\theta}}_{v l}^{(z, k)}>0\right)}}{N} .
$$

The probability of activation in (18) can be computed analogously for (8) and (9) just by taking the appropiate components from $\Theta_{v}^{(z)}$.

\subsection{Forward-filtering-backward-sampling algoritm}

Following the same ideia as in Frühwirth-Schnatter 1994, we now present a matrix-variate version of the forward-filtering-backward-sampling algorithm. It is worth mentioning that in Frühwirth-Schnatter [1994], they use a data augmentation approach in order to tackle the problem related to the estimation of the covariance matrix $\mathbf{W}_{v t}^{(z)}$. Here, instead, we apply a discount factor approach as it is described above. Thus, using the Bayes' theorem and given some assumptions of conditional independence related to the MDLM, it can be shown that

$$
p\left(\mathbf{\Theta}_{v, t-j}^{(z)} \mid \mathbf{\Theta}_{v, t-j+1}^{(z)}, \boldsymbol{\Sigma}^{(z)}, D_{v t}^{(z)}\right) \propto p\left(\mathbf{\Theta}_{v, t-j}^{(z)} \mid \boldsymbol{\Sigma}^{(z)}, D_{v, t-j}^{(z)}\right) p\left(\mathbf{\Theta}_{v, t-j+1}^{(z)} \mid \mathbf{\Theta}_{v, t-j}^{(z)}, \boldsymbol{\Sigma}^{(z)}, D_{v, t-j}^{(z)}\right),
$$

which leads to

$$
\left(\mathbf{\Theta}_{v, t-j}^{(z)} \mid \mathbf{\Theta}_{v, t-j+1}^{(z)}, \boldsymbol{\Sigma}^{(z)}, D_{v t}^{(z)}\right) \sim N_{p q}\left(\mathbf{m}_{v j}^{*(z)}, \mathbf{C}_{v j}^{*(z)}, \mathbf{\Sigma}^{(z)}\right)
$$


where $\mathbf{m}_{v j}^{*(z)}=\mathbf{m}_{v, t-j}^{(z)}+\mathbf{C}_{v, t-j}^{(z)}\left(\mathbf{B}_{\mathbf{t}} \mathbf{C}_{v, t-j}^{(z)} \mathbf{B}_{\mathbf{t}}\right)^{-1}\left(\boldsymbol{\Theta}_{v, t-j+1}^{(z)}-\mathbf{m}_{v, t-j}^{(z)}\right)$ and $\mathbf{C}_{v j}^{*(z)}=\mathbf{C}_{v, t-j}-$ $\mathbf{C}_{v, t-j}\left(\mathbf{B}_{\mathbf{t}} \mathbf{C}_{v, t-j}^{(z)} \mathbf{B}_{\mathbf{t}}\right)^{-1} \mathbf{C}_{v, t-j}$. Thus, the forward-filtering-backward-sampling algorithm for the matrix-variate case is given by:

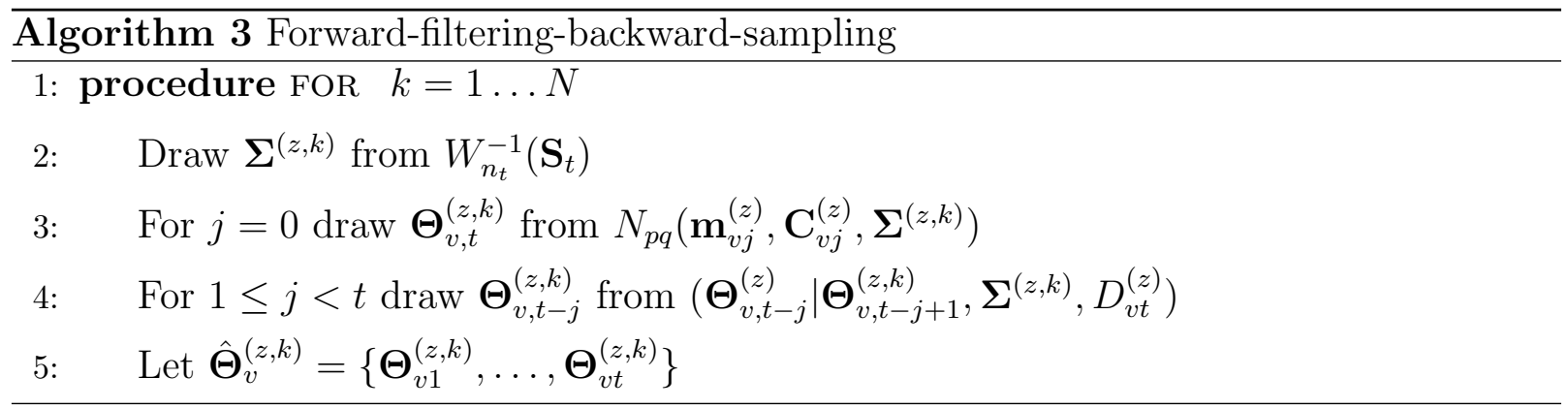

Using Monte Carlo integration as in (18), one can test brain activation just taking the appropiate components from $\boldsymbol{\Theta}_{v}^{(z)}$.

\section{Examples}

In this section we present two applications modeling real fMRI data using model (1) and applying the three algorithms presented above in order to detect brain reaction. In the first example, Pernet et al. 2015] perform an expriment where an auditoy stimulus, following a block-design, was presented in order to localize "temporal voice areas". In that work, Pernet et al. 2015] model the voice and non-voice sounds separate (separately), whereas here, for the sake of simplicity, we merge both vocal and non-vocal sounds in a single block design as it is shown in figure 2. In the second example, Bazán et al. [2015] perform a finger-tapping task experiment, following an event-related design, which should lead to an activation of the motor cortex. With the aim of showing the performance of the three algorithms proposed in this work, we present the analysis for two single voxels (an active and non-active voxel) and activation maps for the entiere brain. In both examples, we run an analysis for one single subject and use the same fix value for the discount factor, i.e. $\beta_{i t}=0.95$ and vague priors (zero mean and large variance) for the state parameter $\boldsymbol{\Theta}_{v 0}^{(z)}$, as well as for $\boldsymbol{\Sigma}_{v}^{(z)}$ with $\boldsymbol{S}_{v o}^{(z)}=\boldsymbol{I}_{q}$ and $n_{0}=1$. 


\section{Auditory cortex activation}

In figure 3, we can see the results after applying the FAST, FSTS and FFBS algorithms to both active and non-active voxels, which are shown in figure 2. Considering that this is an experiment where only sound stimulation is applied, all of the three algorithms yield the results that are expected for voxels located at the temporal cortex and for voxels outside that region.
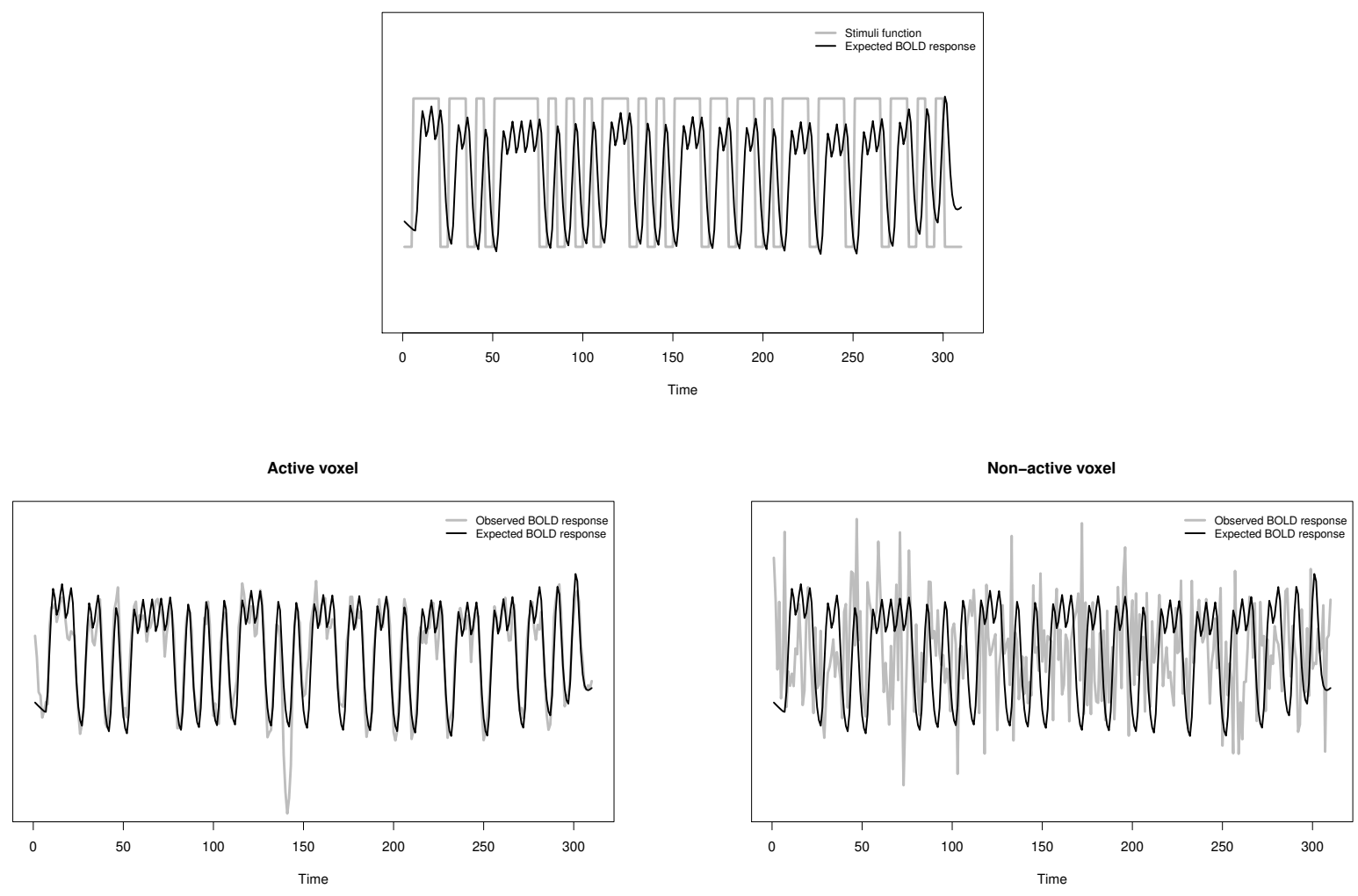

Figure 2: The top figure shows the block-design or stimuli function for voice and non-voice sounds and its expected Bold response. Lower left and right figures show the contrast (or possible match) between the expected and observed bold responses for both an active and non-active voxel respectively on a single subject. 
Active Voxel

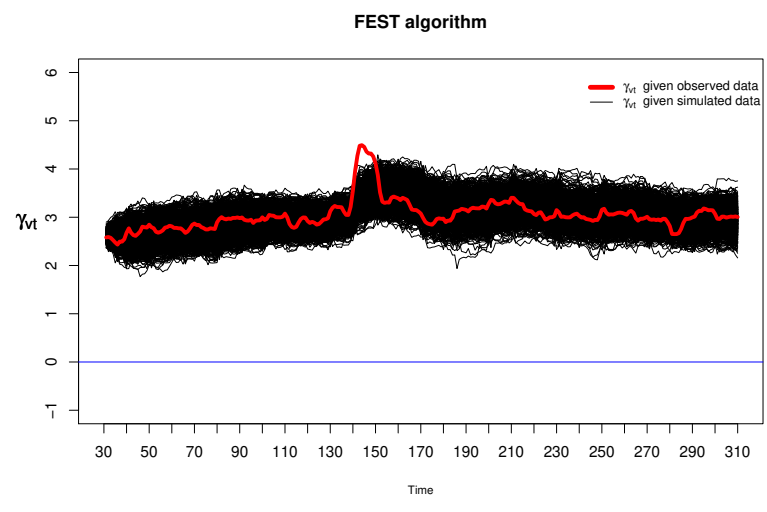

FSTS algorithm

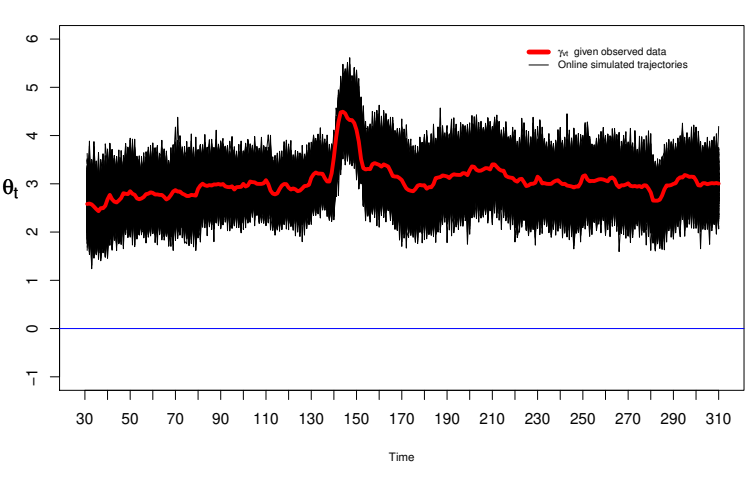

FFBS algorithm

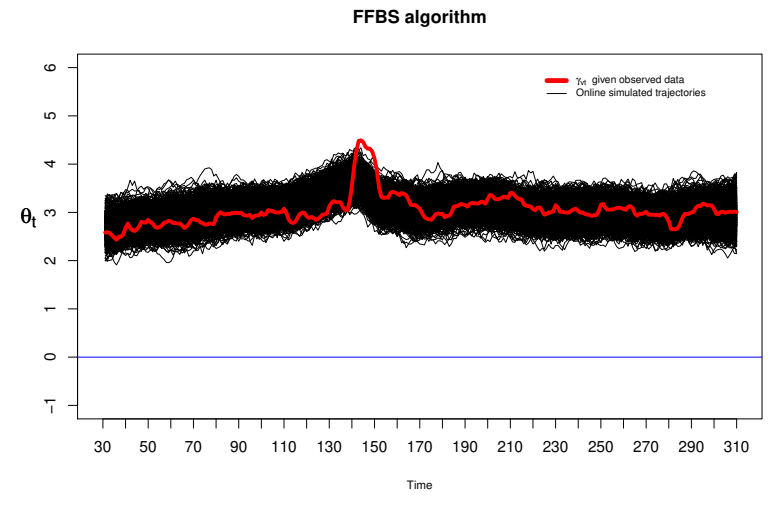

Non-active Voxel

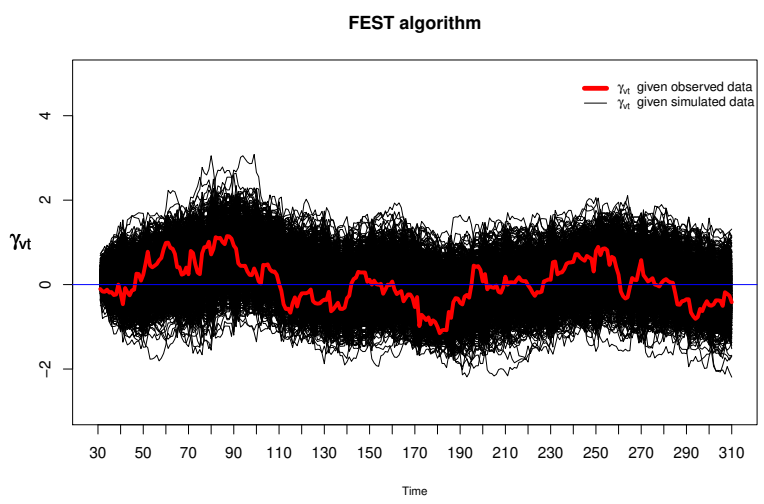

FSTS algorithm

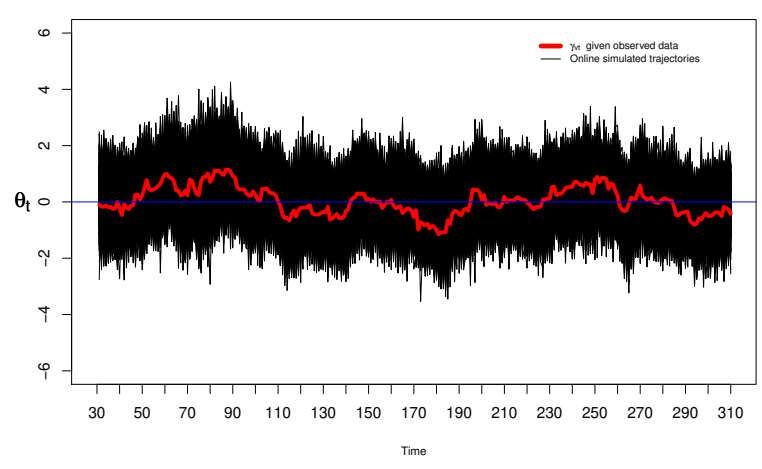

FFBS algorithm

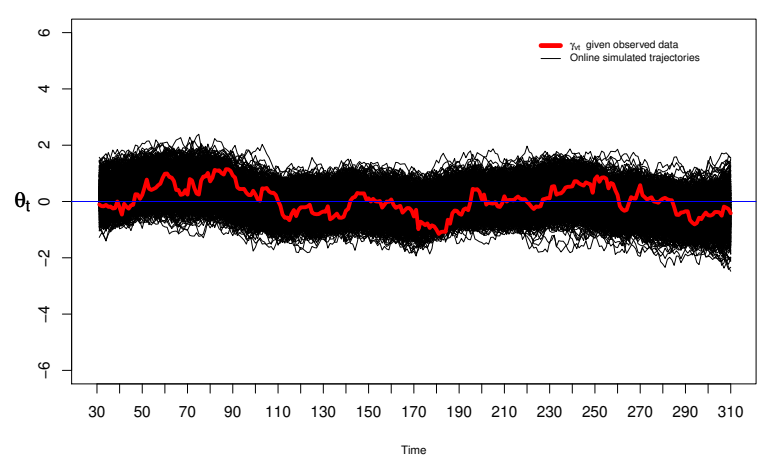

Figure 3: Simulated on-line trajectories obtained when FEST, FSTS, and FFBS algorithms are applied to both active and non-active voxels presented on figure 2 .

For the active voxel, the on-line trajectories related to the state parameter lie above zero, which can be interpreted as a match between the expected and observed bold responses. For non-active voxels, the simulated on-line trajectories lie around zero, which yields lower 
evidence of activation (e.g. < 0.95) according to the Monte Carlo evidence defined on (17) and/or (18). In figure 4, we present the activation maps for a single subject, obtained when applying the FEST, FSTS and FFBS algorithms respectively. For this example, we consider a voxel as active when its computed evidence obtained under any of the three algorithms, is greater than 0.95 . We also present a parametric map obtained when using the General Linear Model (with correction for multiple comparisons using Gaussian randomfield theory and a significant level of $0.01 \%$ ), which can be considered the most common approach employed by users of statistical techniques for fMRI data analysis.

GLM

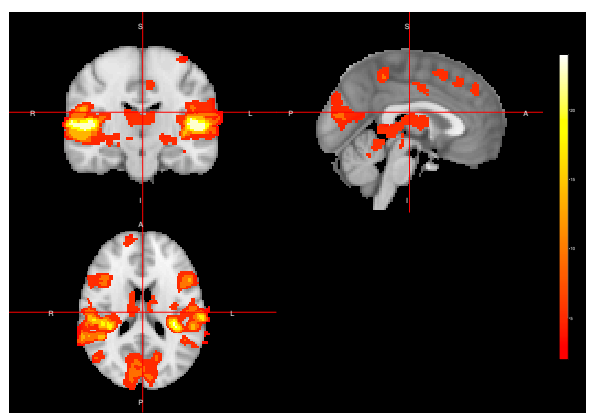

FSTS-ACE

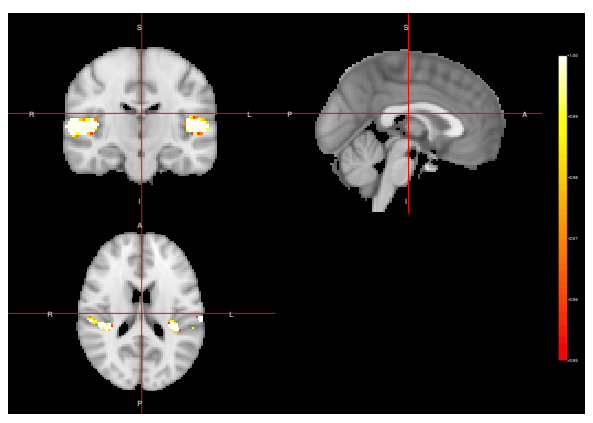

FEST-ACE

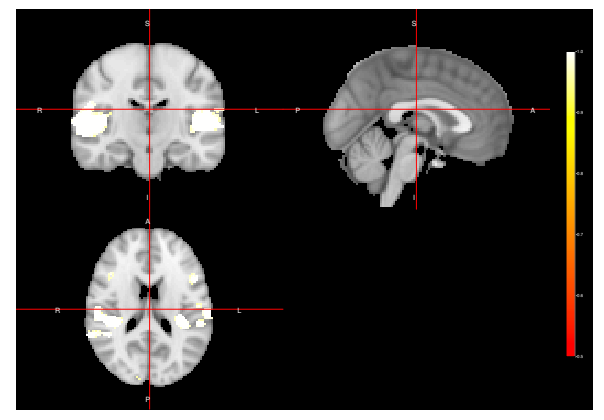

FFBS-ACE

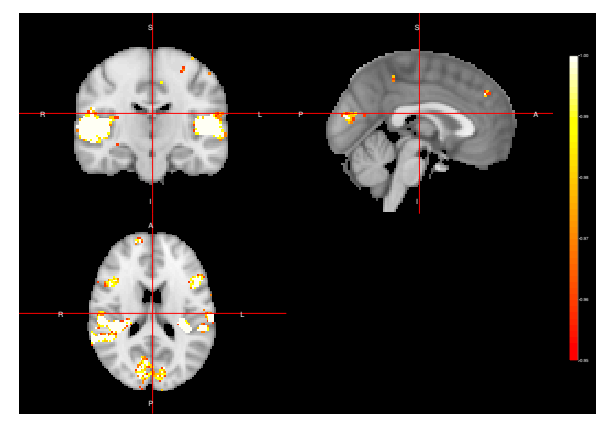

Figure 4: Voxel-wise analysis for one single subject. The top left figure shows a parametric map obtained via General Linear Model (GLM). The top right and lower left and right figures show activation maps related to each of the FEST, FSTS and FFBS algorithms respectively. 
From figures 3 and 4 , we can see the performance of our method in this example, with each of the three algorithms detecting the parietal activation due to sound stimulation. From our knowledge and after evaluating these images with some colleagues from the field of Radiology, we conclude that the FEST algorithm yields better results in terms of a wider parietal activation and less ("false") activations from outside the temporal cortex. FFBS also yields good activation maps, but with more activations from outside the temporal cortex. FSTS also performs well, but shown to be conservative with almost no possible false activations detected and with a smaller parietal activation. For this example, only the average cluster effect (ACE) distribution (9) is employed as the sampler distribution for the state parameter. However, the results are pretty similar when using either the marginal (8) or the joint 10 effect distribution.

\section{Motor cortex activation}
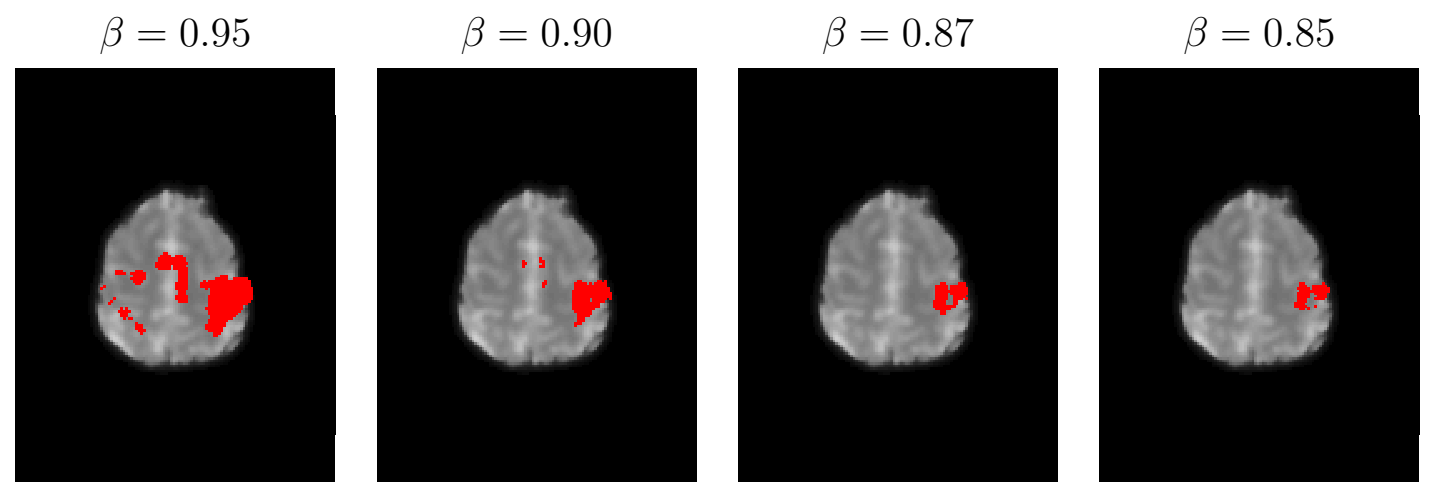

Figure 5: Activation maps of 95\% obtained using the FEST-ACE algorithm for different values of the discount factor $\beta$.

In figure 5, we can see an activation map corresponding to the event-related finger-tapping experiment from Bazán et al. [2015] obtained when using the FEST algorithm. When the FSTS and FFBS algorithms are applied, they yield pretty similar results successfully identifying the motor cortex activation expected from this experiment. What we aiming to highlight with this example is the key role played by the discount parameter $\beta$. In the 
modeling we are proposing here, we let the value of $\beta$ as a free input value defined by the user. However, we can see that the activation map is sensitive to the value set for $\beta$. From our empirical experience, we recommend values for $0.8<\beta<1$. One possible way to overcome this limitation is to run several maps for different $\beta$ values and use a statistical criterion (e.g. Bayes factor) to select the "appropriate" map. In the final section, we discuss this issue in more detail.

\section{Assessment of the Method}

In order to assess the activation maps obtained when the model (1) is fitting and any of the algorithms proposed in this work is used to detect voxel activation, we employ two different approaches. For the first type of assessment, we resort to simulated data using the R package neuRosim (Welvaert et al. [2011]). The second assessment approach is inspired by the work of Eklund et al. [2016], where real data from resting state experiments is used in order to evaluate the rate of false positive activations in the entire brain.

\subsection{Using simutaled data}

The aim of using simulated data is to evaluate the capacity of our method to detect true voxel activations, which in this case are artificially created using the $\mathrm{R}$ package neuRosim. Specifically, we create two spherical shape activations in the visual cortex related to a block design experiment. In Welvaert et al. 2011 the interested reader can find a detailed explanation on how to simulate fMRI data using the neuRosim package. In this aeessment, we set two different radius sizes (4 and 7 respectively) and the same effect size (250) for the two activated regions. In the figure 6, we can see the activation maps obtained when using each one from the three algorithms FEST, FSTS, and FFBS, for two different levels of signal-to-noise ratio (SNR). From figure 6, we can see that when a high SNR (30) is fixed, all the three algorithms successfully identify the activated regions. However, when a low SNR (3.2) is set the FSTS algorithm fails to identify the activated regions, even when a less conservative probability threshold is applied (90\%). The activation maps, when using FEST and FFBS algorithms under both scenarios yield quite good results, with a notorious 
performance of the FEST algorithm in terms of none false-positive activations.

$$
\mathrm{SNR}=30
$$
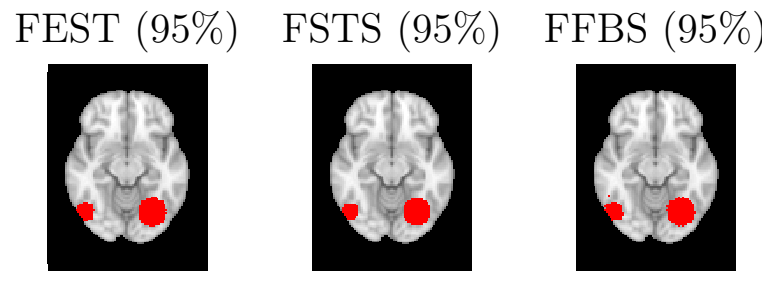

$$
\mathbf{S N R}=3.2
$$
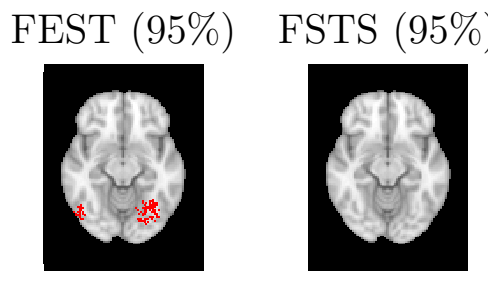

FFBS (95\%)
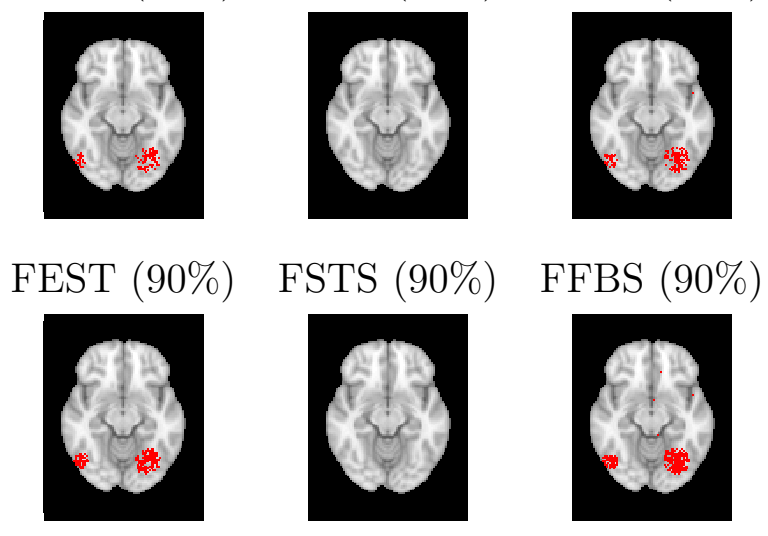

Figure 6: activation maps for fMRI simulated data under different SNR levels, algorithms (FEST, FFBS and FFBS), and probability activation thresholds.

\subsection{Using real data}

One of the big challenges to the statistical techniques for fMRI data analysis is controling the rate of false-positve activations, which usually appear on activation maps. For instance, in the case of the GLM, even using some sort of corrections for multiple comparisons, the rate of false-positive can be as high as 70\% (Eklund et al. [2012] and Eklund et al. [2016]). Thus, in order to assess the capacity of the method proposed in this work related to the control of false activations, we follow the same approach as in Eklund et al. 2016. That approach consists on the use of real fMRI data, related to resting-state experiments obtained from the 1000 Functional Connectomes Project Biswal et al. [2010], which are 
correlated with covariates from fictitious stimuli. Hence, all the significant activations detected are interpreted as false-positive activations. This is a resaonable assumption in the sense that if one models the observed BOLD signal $\left(Y_{v t}^{(z)}\right)$ from a resting-state experiment as $E\left(Y_{v t}^{(z)}\right)=\mathbf{F}_{v t}^{\prime} \boldsymbol{\Theta}_{v t}$, where $\mathbf{F}_{v t}^{\prime}$ is an (fictitious) expected BOLD signal not related with $Y_{v t}^{(z)}$, what one would expect to find is $\left.E\left(\boldsymbol{\Theta}_{v t} \mid D_{v t}\right)\right) \leq 0$, which can be interpreted as no existing match between the expected and observed BOLD signals. Therefore, we create four fictitious variables related to two block paradigms (B1 (10-s on off), B2 (30s on off)) and to two event-related paradigms (E1 (2-s activation, 6-rest), E2(1- to 4-s activation, 3to $6 \mathrm{~s}$ rest, randomized)). We correlate those variables with the fMRI resting data from three different labs (Beijing(198 subjects), Cambridge (198 subjects), Oulu (103 subjects)), and count the number of (false) significant activations. This analysis is only performed at the individual level.

Table 1: Rate of false-positive activations for the fictitious block paradigms B1 and B2.

\begin{tabular}{|c|c|c|c|c|c|c|c|c|c|}
\hline \multicolumn{10}{|c|}{ B1 } \\
\hline \multirow[b]{2}{*}{ Source fMRI data } & \multicolumn{3}{|c|}{ FEST } & \multicolumn{3}{|c|}{ FSTS } & \multicolumn{3}{|c|}{ FFBS } \\
\hline & Marginal & LTT & Joint & Marginal & LTT & Joint & Marginal & LTT & Joint \\
\hline Beijing (198 subjects) & 0.0 & 0.0 & 0.0 & 0.0 & 0.0 & 0.0 & $2.5 \times 10^{-3}$ & $4.1 \times 10^{-3}$ & $6.7 \times 10^{-6}$ \\
\hline Cambridge (198 subjects) & $7.9 \times 10^{-5}$ & $1.1 \times 10^{-4}$ & 0.0 & 0.0 & 0.0 & 0.0 & $3.9 \times 10^{-3}$ & $5.4 \times 10^{-3}$ & $1.4 \times 10^{-5}$ \\
\hline Oulu (103 subjects) & 0.0 & 0.0 & 0.0 & 0.0 & 0.0 & 0.0 & $1.4 \times 10^{-4}$ & $3.2 \times 10^{-4}$ & 0.0 \\
\hline \multicolumn{10}{|c|}{ B2 } \\
\hline & \multicolumn{3}{|c|}{ FEST } & \multicolumn{3}{|c|}{ FSTS } & \multicolumn{3}{|c|}{ FFBS } \\
\hline Source fMRI data & Marginal & LTT & Joint & Marginal & LTT & Joint & Marginal & LTT & Joint \\
\hline Beijing (198 subjects) & $3.1 \times 10^{-6}$ & $5.7 \times 10^{-6}$ & 0.0 & 0.0 & 0.0 & 0.0 & $7.0 \times 10^{-3}$ & $1.2 \times 10^{-2}$ & $1.3 \times 10^{-4}$ \\
\hline Cambridge (198 subjects) & $1.4 \times 10^{-3}$ & $2.1 \times 10^{-3}$ & $2.0 \times 10^{-5}$ & $6.70 \times 10^{-8}$ & $1.53 \times 10^{-5}$ & $2.9 \times 10^{-5}$ & $9.9 \times 10^{-3}$ & $1.4 \times 10^{-2}$ & $2.1 \times 10^{-4}$ \\
\hline Oulu (103 subjects) & 0.0 & 0.0 & 0.0 & 0.0 & 0.0 & 0.0 & $1.2 \times 10^{-2}$ & $2.1 \times 10^{-2}$ & $4.9 \times 10^{-4}$ \\
\hline
\end{tabular}

In the table 1, we only show the assessment results for the variables B1 and B2 given that E1 and E2 yield pretty similar outcomes. Those results are congruent with what is observed in the assessmente with simulated data and real data examples shown above. The three algorithms present a low rate of false-positive activations, but with a less conservative behavior in the case of FEST and FFBS allowing for the detection of weaker BOLD signals. From these assessments (using real and simulated data), the examples presented above and other analysis that are not possible to be presented here, we would recommend the FEST as the best option among the three algorithms, not only because its good performance related to weak BOLD signals and false-positive activations, but also because its time of 
execution is $50 \%$ faster in comparison with the FFBS and FSTS algorithms.

\section{Concluding remarks}

In this work we develop an effective and reliable method for statistical analysis of fMRI data, taking into account its temporal and (at least locally) spatial features, without the need for the more traditional and complex spatial-temporal models. We employ an MDLM, which has shown to be a very flexible type of modeling beacuse of the availability of closed-form posterior distributions, discount factors to deal with the variability of the state parameter and the possibility to combine the posterior distributions through subjects to perform group analysis. Another important aspect of the MDLM is its relationship with the traditional and broadly known GLM ([West and Harrison, 1997, page 80]), which can help to nonstatistician practitioners to get more familiar with this type of modeling. We also make an important contribution, not only in the context of fMRI modeling but a general way to perform an assessment of dynamic effects on Dynamic Linear Models. From our knowledge, this is something that has been neglected, as it is mentioned above, possibly because of the type of applications and motivations behind MDLM. In this work, instead of making predictions or analyze covariance structures among time series, our aim is to assess affects size related to neural activity. Thus in order to achieve that using this type of modeling, we propose two new algorithms (FEST, FSTS) and adapt an existing one (FBST) to draw online trajectories related to the state parameter. Throughout the examples and assessments presented above, one can see that the performance of our method is remarkable, in the sense that it is able to both properly identify brain activation under different conditions of experimentation and control the rate of false activations. Currently we are working on the implementation of an $\mathrm{R}$ package for this method, which will be freely available for interested users from the fMRI community. It is worth mentioning that with this work we leave some open problems. One of them is the choice of the discount factor $\beta$, which from our experience dealing with fMRI data from different types of experiments just setting values for $0.8<\beta<1$ can work well. But instead of setting or tuning a particular value for $\beta$, a model selection approach for different $\beta$ values could be easily implemented in order to select the "best" $\beta$ value according to some statistical criteria such as Bayes 
Factors. Another problem is related to the distance parameter $r$, for which we believe a sensitivity analysis could be performed in order to have a better sense of the behavior of this relevant parameter. In all of the examples and analysis performed in this work, we set $r=1$. Aditionally, in the case of the FEST algorithm, there are some outputs such as

the sequence of covariance matrices $\left\{\mathbf{S}_{v 1}^{(z, k)}, \ldots, \mathbf{S}_{v t}^{(z, k)}\right\}$, which could be used to build some sort of dynamic graphs to tackle the connectivity problem among brain regions for restingstate fMRI data (see Shen et al. 2010] for a better understanding of this type of analysis). Finally, we want to highlight that in this work only a tiny part of the rich possibilities offered by the general theory of the DLM's is explored. Our ideas could be extended to more complex model specifications or even to the case of non-linear Dynamic models.

\section{References}

Russell A Poldrack, Jeanette A Mumford, and Thomas E Nichols. Handbook of functional MRI data analysis. Cambridge University Press, 2011.

Anders Eklund, Thomas E Nichols, and Hans Knutsson. Cluster failure: why fmri inferences for spatial extent have inflated false-positive rates. Proceedings of the National Academy of Sciences, 113(28):7900-7905, 2016.

Linlin Zhang, Michele Guindani, Francesco Versace, Jeffrey M Engelmann, Marina Vannucci, et al. A spatiotemporal nonparametric bayesian model of multi-subject fmri data. The Annals of Applied Statistics, 10(2):638-666, 2016.

Anders Eklund, Martin A Lindquist, and Mattias Villani. A bayesian heteroscedastic glm with application to fmri data with motion spikes. NeuroImage, 155:354-369, 2017.

Martin Bezener, John Hughes, Galin Jones, et al. Bayesian spatiotemporal modeling using hierarchical spatial priors, with applications to functional magnetic resonance imaging. Bayesian Analysis, 2018. 
Cheng-Han Yu, Raquel Prado, Hernando Ombao, and Daniel Rowe. A bayesian variable selection approach yields improved detection of brain activation from complex-valued fmri. Journal of the American Statistical Association, (just-accepted):1-61, 2018.

Linlin Zhang, Michele Guindani, and Marina Vannucci. Bayesian models for fmri data analysis. Wiley interdisciplinary reviews. Computational statistics, 7(1):21-41, 2015.

F DuBois Bowman. Brain imaging analysis. Annual review of statistics and its application, 1:61-85, 2014.

Mike West and Jeff Harrison. Bayesian Forecasting and Dynamic Models (2Nd Ed.). Springer-Verlag New York, Inc., New York, NY, USA, 1997. ISBN 0-387-94725-6.

Jose Mario Quintana. A dynamic linear matrix-variate regression model, 1985.

Jose Mario Quintana. Multivariate Bayesian forecasting models. PhD thesis, University of Warwick, 1987.

Nasser H Kashou. A practical guide to an fmri experiment. in: Duric d, editor. advanced brain neuroimaging topics in health and disease - methods and applications. 2014.

Jose Mario Quintana and Mike West. An analysis of international exchange rates using multivariate dlm's. The Statistician, pages 275-281, 1987.

Omar Aguilar and Mike West. Bayesian dynamic factor models and portfolio allocation. Journal of Business 6 Economic Statistics, 18(3):338-357, 2000.

Xiang Fei, Chung-Cheng Lu, and Ke Liu. A bayesian dynamic linear model approach for real-time short-term freeway travel time prediction. Transportation Research Part C: Emerging Technologies, 19(6):1306-1318, 2011.

Sylvia Frühwirth-Schnatter. Data augmentation and dynamic linear models. Journal of time series analysis, 15(2):183-202, 1994.

JR Ameen and P.J Harrison. Normal discount bayesian models. In Bayesian Statistics 2: Proceedings of the Second Valencia International Meeting, September 6/10, 1985, volume 2, pages 271-298. North Holland, 1985. 
Bharat B Biswal, Maarten Mennes, Xi-Nian Zuo, Suril Gohel, Clare Kelly, Steve M Smith, Christian F Beckmann, Jonathan S Adelstein, Randy L Buckner, Stan Colcombe, et al. Toward discovery science of human brain function. Proceedings of the National Academy of Sciences, 107(10):4734-4739, 2010.

Marijke Welvaert, Joke Durnez, Beatrijs Moerkerke, Geert Verdoolaege, and Yves Rosseel. neurosim: An r package for generating fmri data. Journal of Statistical Software, 44(10): $1-18,2011$.

A Philip Dawid. Some matrix-variate distribution theory: notational considerations and a bayesian application. Biometrika, 68(1):265-274, 1981.

William D Penny, Karl J Friston, John T Ashburner, Stefan J Kiebel, and Thomas E Nichols. Statistical parametric mapping: the analysis of functional brain images. Elsevier, 2011.

Mike West, P Jeff Harrison, and Helio S Migon. Dynamic generalized linear models and bayesian forecasting. Journal of the American Statistical Association, 80(389):73-83, 1985.

R Core Team. R: A Language and Environment for Statistical Computing. R Foundation for Statistical Computing, Vienna, Austria, 2018. URL https://www.R-project.org/.

Dirk Eddelbuettel and Conrad Sanderson. Rcpparmadillo: Accelerating $\mathrm{r}$ with highperformance c++ linear algebra. Computational Statistics and Data Analysis, 71:10541063, March 2014. URL http://dx.doi.org/10.1016/j.csda.2013.02.005.

Brandon Whitcher, Volker J. Schmid, and Andrew Thornton. Working with the DICOM and NIfTI data standards in R. Journal of Statistical Software, 44(6):1-28, 2011. URL http://www.jstatsoft.org/v44/i06/.

John Muschelli. neurobase: 'Neuroconductor' Base Package with Helper Functions for 'nifti' Objects, 2018. URL https://CRAN.R-project.org/package=neurobase. R package version 1.27.6. 
Mark Jenkinson, Christian F Beckmann, Timothy EJ Behrens, Mark W Woolrich, and Stephen M Smith. Fsl. Neuroimage, 62(2):782-790, 2012.

Cyril R Pernet, Phil McAleer, Marianne Latinus, Krzysztof J Gorgolewski, Ian Charest, Patricia EG Bestelmeyer, Rebecca H Watson, David Fleming, Frances Crabbe, Mitchell Valdes-Sosa, et al. The human voice areas: Spatial organization and inter-individual variability in temporal and extra-temporal cortices. Neuroimage, 119:164-174, 2015.

Paulo Rodrigo Bazán, Claudinei Eduardo Biazoli Jr, Joao Ricardo Sato, and Edson Amaro Jr. Motor readiness increases brain connectivity between default-mode network and motor cortex: impact on sampling resting periods from fmri event-related studies. Brain connectivity, 5(10):631-640, 2015.

Anders Eklund, Mats Andersson, Camilla Josephson, Magnus Johannesson, and Hans Knutsson. Does parametric fmri analysis with spm yield valid results?an empirical study of 1484 rest datasets. NeuroImage, 61(3):565-578, 2012.

Xilin Shen, Xenophon Papademetris, and R Todd Constable. Graph-theory based parcellation of functional subunits in the brain from resting-state fmri data. Neuroimage, 50 (3):1027-1035, 2010. 


\title{
fMRI group analysis based on outputs from Matrix-Variate Dynamic Linear Models.
}

\author{
January 16, 2020
}

\begin{abstract}
In this work, we describe in more detail how to perform fMRI group analysis using inputs from modeling fMRI signal using Matrix-Variate Dynamic Linear Models (MDLM) at the individual level. After computing a posterior distribution for the average group activation, the three algorithms (FEST, FSTS, and FFBS) proposed from our previous work [Jiménez et al., 2019] can be easily implemented. We also propose an additional algorithm, which we call AG-algorithm, to draw on-line trajectories of the state parameter and therefore assess voxel activation at the group level. The performance of our method is illustrated through one practical example using real fMRI data from a "voice-localizer" experiment.
\end{abstract}

Keywords: fMRI, Bayesian Analysis, Matrix-Variate Dynamic Linear Models, Monte Carlo Integration.

\section{Introduction}

Statistical fMRI group analysis is a procedure commonly used by practitioners in the neuroscience community. One of the aims of this type of analysis can be either identify patterns of brain activation for a specific group of individuals who suffer some types of illnesses (or conditions) or just for the general understanding of the functioning of the human brain under specific types of stimuli. One example of the former is the study performed by Batistuzzo et al. [2015], where an fMRI group analysis is performed in order to characterized prefrontal activation under specific experimental conditions in pediatric patients who suffer from obsessive-compulsive disorders. On the other hand, one good example of studies which explores mechanisms of human brain activation is the work developed by 
Pernet et al. [2015], where an fMRI group analysis is performed in order to characterize specific areas of the temporal cortex involved in the perception of the human voice. Those are just two examples, from thousands of studies that have been published since the arrival of the MRI scanners. To carry on this type of fMRI group analysis there are some packages available, such as FSL [Jenkinson et al., 2012] and SPM [Penny et al., 2011], with implementations of different types of statistical analysis. The most common statistical technique reported in the majority of fMRI studies is the so-called General Linear Model (GLM), which is noting more than a normal linear model. The GLM is used only at the individual stage, where it is fitted on every voxel (voxel-wise approach) for every subject in the sample, and its estimated size effects (and sometimes its standard errors as well) from the covariates (usually related with the stimuli presented on the experiment) are used as an input product for the group stage. In this work, we present a similar approach, but instead of a GLM, we use a Matrix-Variate Dynamic Linear Model (MVDLM) in order to incorporate the temporal and spatial structures usually present in fMRI data. The description of the modeling at the individual stage is presented in paper $\mathbf{1}^{1}$. In this work, we mainly focus on the description of the group stage analysis assuming that we already have available the posterior distributions obtained at the individual stage.

In the next section, we present the statistical procedure to both detections of brain activation patterns from one single group and the comparison of activation strengths between two groups. In section 3, we present one practical example from an experiment exploring brain activation in the temporal cortex. Finally, in section 4 some concluding remarks are presented.

\section{2 fMRI Group Analysis}

Let's suppose we have $N_{g}$ subjects in $g$ different groups, for which there is a need to explore and/or characterized some aspects related to their brain functioning. The most common cases in the fMRI literature are for $g=1,2$, when either there is an interest for the pattern of activation from a single group or the comparison of activation strength between groups

\footnotetext{
${ }^{1}$ Assessing Dynamic Effects on a Bayesian Matrix-Variate Dynamic Linear Model: an Application to fMRI Data Analysis.
} 
cases and controls. Suppose also that the model (1) is fitted at the voxel level for every subject from each group as it is performed in Jiménez et al. [2019].

$$
\begin{array}{ll}
\text { Observation: } & \mathbf{Y}_{v t}^{(z)}=\mathbf{F}_{t}^{\prime} \boldsymbol{\Theta}_{v t}^{(z)}+\boldsymbol{\nu}_{v t}^{\prime(z)} \\
\text { Evolution: } & \boldsymbol{\Theta}_{v t}^{(z)}=\mathbf{G}_{t} \boldsymbol{\Theta}_{v t-1}^{(z)}+\boldsymbol{\Omega}_{v t}^{(z)},
\end{array}
$$

where $v=[i, j, k]$ represent the voxel position in the brain image and $z=1, \ldots, N_{g}$. Thus, the posterior distribution $p\left(\boldsymbol{\Theta}_{v t}^{(z)} \mid \mathbf{Y}_{v t}^{(z)}\right)$ is given by

$$
\left(\boldsymbol{\Theta}_{v t}^{(z)} \mid D_{v t}^{(z)}\right) \sim T_{n_{t}}\left[\mathbf{m}_{v t}^{(z)}, \mathbf{C}_{v t}^{(z)}, \mathbf{S}_{t v}^{(z)}\right]
$$

In the contex of this modeling the $p \times q$ parameter $\boldsymbol{\Theta}_{v t}^{(z)}$ brings information about the brain activation for subject $z$, at position $v$ and time $t$. Specifically, when $\left\{\boldsymbol{\Theta}_{v t}^{(z)}>0\right\}$ it is interpreted as a match between the expected (the components of $\mathbf{F}_{t}^{\prime}$ ) and observed BOLD $\left(\mathbf{Y}_{v t}^{(z)}\right)$ responses, which simply means a brain activation. In that sense, we would like to combine that information among the subjects and compute a new measure which would inform about the activation pattern of the entire group. Thus, in order to do so, we follow a similar approach as in Beckmann et al. [2003], where $\boldsymbol{\Theta}_{v t}^{(g)}=\frac{1}{N_{g}} \sum_{z} \boldsymbol{\Theta}_{v t}^{(z)}$ represents the average group activation at positon $v$ and time $t$. However, given that the distribution of the original variables is matrix $\mathrm{T}$ distribution, dealing with the resulting distribution from the new variable $\boldsymbol{\Theta}_{v t}^{(g)}$ can be cumbersome. Thus, in order to overcome this problem, we take advantage of this reasonable approximation: the posterior distribution of $\boldsymbol{\Theta}_{v t}^{(z)}$ when $n_{t} \geq 30$ can be represented by

$$
\left(\Theta_{v t}^{(z)} \mid D_{v t}^{(z)}\right) \stackrel{\text { approx }}{\sim} N_{p q}\left[\mathbf{m}_{v t}^{(z)}, \mathbf{C}_{v t}^{(z)}, \mathbf{S}_{v t}^{(z)}\right]
$$

Then, from the properties of the matrix variate distribution [Gupta and Nagar, 1999], the distribution of the average group activation is given by

$$
\left(\mathbf{\Theta}_{v t}^{(g)} \mid D_{v t}^{(g)}\right) \stackrel{a p p r o x}{\sim} N_{p q}\left[\frac{1}{N_{g}} \sum_{z} \mathbf{m}_{v t}^{(z)}, \frac{1}{N_{g}^{2}} \sum_{z} \mathbf{C}_{v t}^{(z)}, \frac{1}{N_{g}^{2}} \sum_{z} \mathbf{S}_{v t}^{(z)}\right]
$$

Having the distribution (4) available, the group activation at voxel $v$ can be assessed by using any of the algorithms we define in Jiménez et al. [2019]. From the posterior distribu- 
tion (4), we define three different probability distributions obtained from the components of $\boldsymbol{\Theta}_{v t}^{(g)}$ :

$$
\text { Marginal group effect: } \bar{\theta}_{v t, l}^{*(g)} \sim N\left(\bar{m}_{v t, l}^{*(g)}, \bar{S}_{v t, l}^{(g)}\right)
$$

Average cluster group effect: $\overline{\bar{\theta}}_{v t, l}^{(g)} \sim N\left(\overline{\bar{m}}_{v t, l}^{(g)}, \overline{\bar{S}}_{t, l}^{(g)}\right)$,

$$
\text { Joint group effect: } \overline{\boldsymbol{\theta}}_{v t, l}^{(g)} \sim N_{q}\left(\overline{\boldsymbol{m}}_{v t, l}^{(g)}, \overline{\boldsymbol{S}}_{t}^{(g)}\right) \text {, }
$$

where $l=1, \ldots, p$. In [Jiménez et al., 2019], the interested reader can find a more detailed explanation on how distributions (5), (6) and (7) are obtained.

\section{Algorithms for Group Analysis}

In this section we present the versions for group analysis of the FEST, FSTS and FFBS algorithms. We also present an aditional algorithm, which rely on outputs from those three algorithms at the individual level.

\section{FEST algorithm}

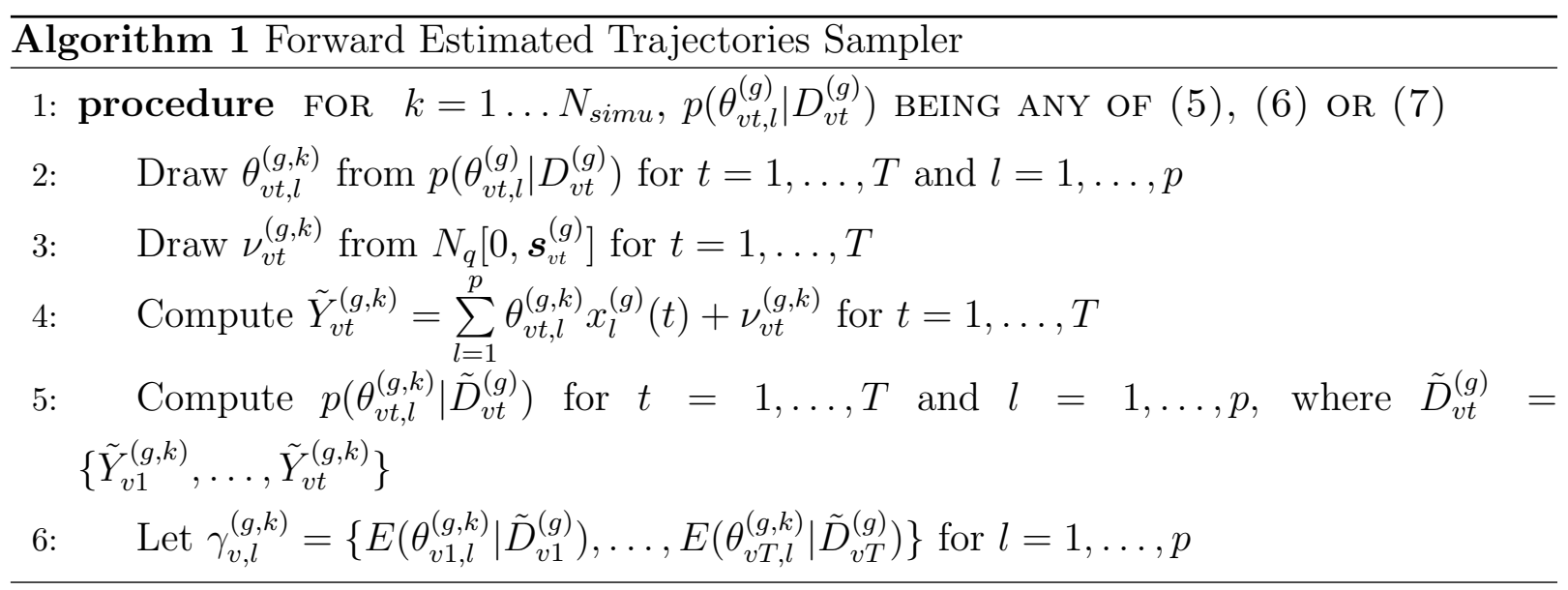

Thus, a measure of evidence for group activation related to stimulus $l$ at position $v$ is 
given by

$$
p\left(\gamma_{v, l}^{(g)}>0\right)=E\left(\mathbb{1}_{\left(\gamma_{v, l}^{(g)}>0\right)}\right) \approx \frac{\sum_{k=1}^{N_{\text {simu }}} \mathbb{1}_{\left(\gamma_{v, l}^{(g, k)}>0\right)}}{N_{\text {simu }}} .
$$

One limitation of this algorithm is that it depends on $x_{l}^{(g)}(t)$ being the same for all the subjects. Despite most of the fMRI group experiment meeting this requirement, there are some designs where random sequences of stimuli are presented, so in cases like these, the form of the expected BOLD response $\left(x_{l}^{(g)}(t)\right)$ will be different among the subjects. The FSTS and FFBS algorithms can help to overcome this specific limitation since they do not depend on $x_{l}^{(g)}(t)$.

\section{FSTS algorithm}

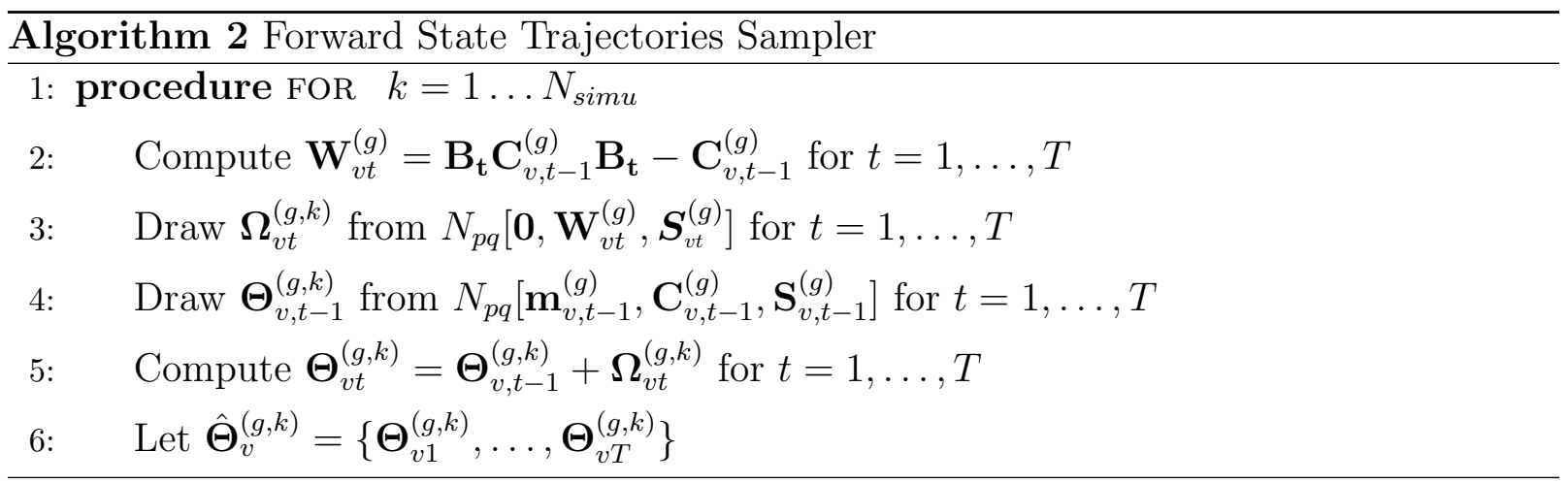

Using Monte Carlo integration as in (10), one can test brain activation just by taking the appropriate components from $\hat{\mathbf{\Theta}}_{v}^{(g, k)}$ to compute whichever marginal, average or joint effects.

\section{FFBS algorithm}

The FFBS algorithm at the individual level rely on the filtered distribution $p\left(\Theta_{v, t-j}^{(z)} \mid \Theta_{v, t-j+1}^{(z)}, \boldsymbol{\Sigma}^{(z)}, D_{v t}^{(z)}\right)$, which under the assumptions and prior distributions set for model (1) is given by

$$
N_{p q}\left(\mathbf{m}_{v j}^{*(z)}, \mathbf{C}_{v j}^{*(z)}, \Sigma^{(z)}\right)
$$

In order to define the FFBS algorithm for the group case, we just adapt the distribution (9) by replacing its parameters $\mathbf{m}_{v j}^{*(z)}, \mathbf{C}_{v j}^{*(z)}$ and $\boldsymbol{\Sigma}^{(z)}$ for $\mathbf{m}_{v j}^{*(g)}, \mathbf{C}_{v j}^{*(g)}$ and $\boldsymbol{\Sigma}^{(g)}$ respectively. 
In this way, the adapted filtered distribution for the group case is given by

$$
\left(\boldsymbol{\Theta}_{v, t-j}^{(g)} \mid \Theta_{v, t-j+1}^{(g)}, \boldsymbol{\Sigma}^{(g)}, D_{v t}^{(g)}\right) \sim N_{p q}\left(\mathbf{m}_{v j}^{*(g)}, \mathbf{C}_{v j}^{*(g)}, \boldsymbol{\Sigma}^{(g)}\right)
$$

where $\mathbf{m}_{v j}^{*(g)}=\mathbf{m}_{v, t-j}^{(g)}+\mathbf{C}_{v, t-j}^{(g)}\left(\mathbf{B}_{\mathbf{t}} \mathbf{C}_{v, t-j}^{(g)} \mathbf{B}_{\mathbf{t}}\right)^{-1}\left(\boldsymbol{\Theta}_{v, t-j+1}^{(g)}-\mathbf{m}_{v, t-j}^{(g)}\right)$ and $\mathbf{C}_{v j}^{*(g)}=\mathbf{C}_{v, t-j}^{(g)}-$ $\mathbf{C}_{v, t-j}^{(g)}\left(\mathbf{B}_{\mathbf{t}} \mathbf{C}_{v, t-j}^{(g)} \mathbf{B}_{\mathbf{t}}\right)^{-1} \mathbf{C}_{v, t-j}^{(g)}$.

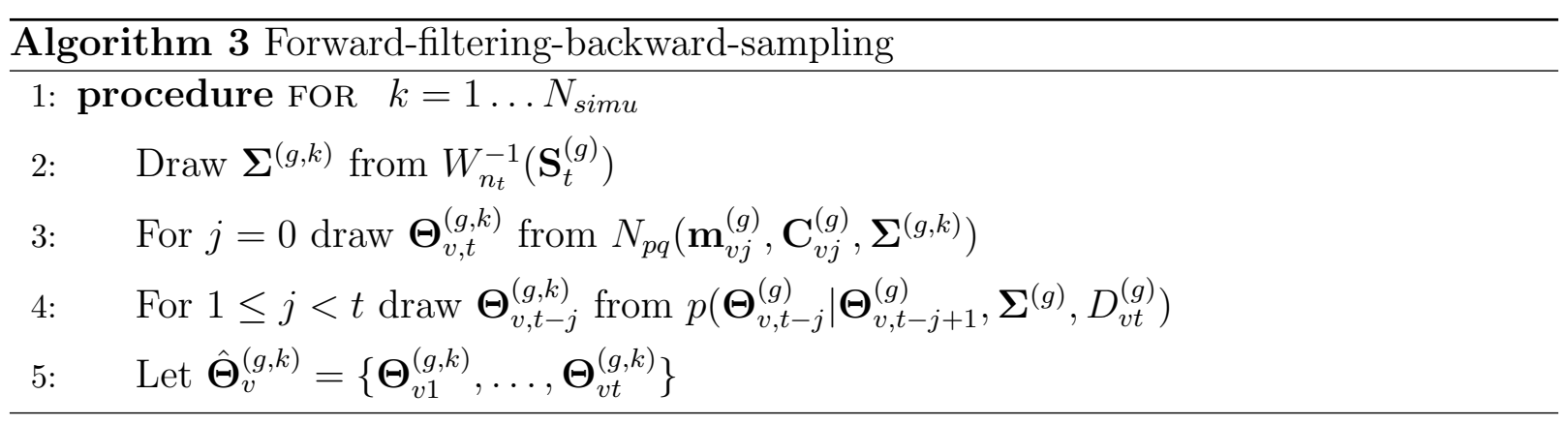

From the simulated on-line trajectories $\hat{\Theta}_{v}^{(g, k)}$ one can compute an activation evidence as in the case of the FSTS algorithm.

\section{AG-algorithm}

Average Group algorithm (AG-algorithm) is just another way to simulate on-line trajectories of the state parameter, which are used to assess voxel activation at the group level. However, instead of using the distribution of the average group effect (4), the AG-algorithm samples on-line estimated trajectories at the individual level and then compute an average group on-line trajectory at voxel $v$. What is intended with this approach is to allow more variability among subjects to be taken into account when computing group activation effects.

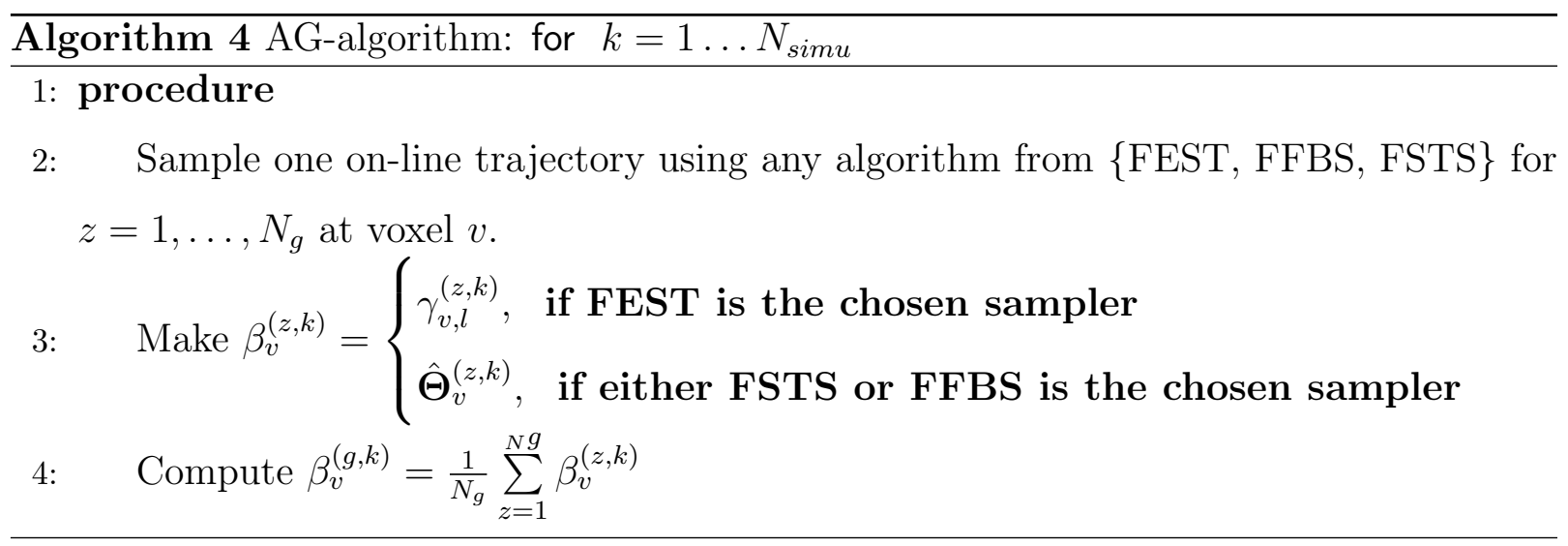


Therefore, a measure of evidence for group activation at voxel $v$ is given by:

$$
p\left(\beta_{v}^{(g)}>0\right)=E\left(\mathbb{1}_{\left(\beta_{v}^{(g)}>0\right)}\right) \approx \frac{\sum_{k=1}^{N_{\text {simu }}} \mathbb{1}_{\left(\beta_{v}^{(g, k)}>0\right)} .}{N_{\text {simu }}} .
$$

\section{Example}

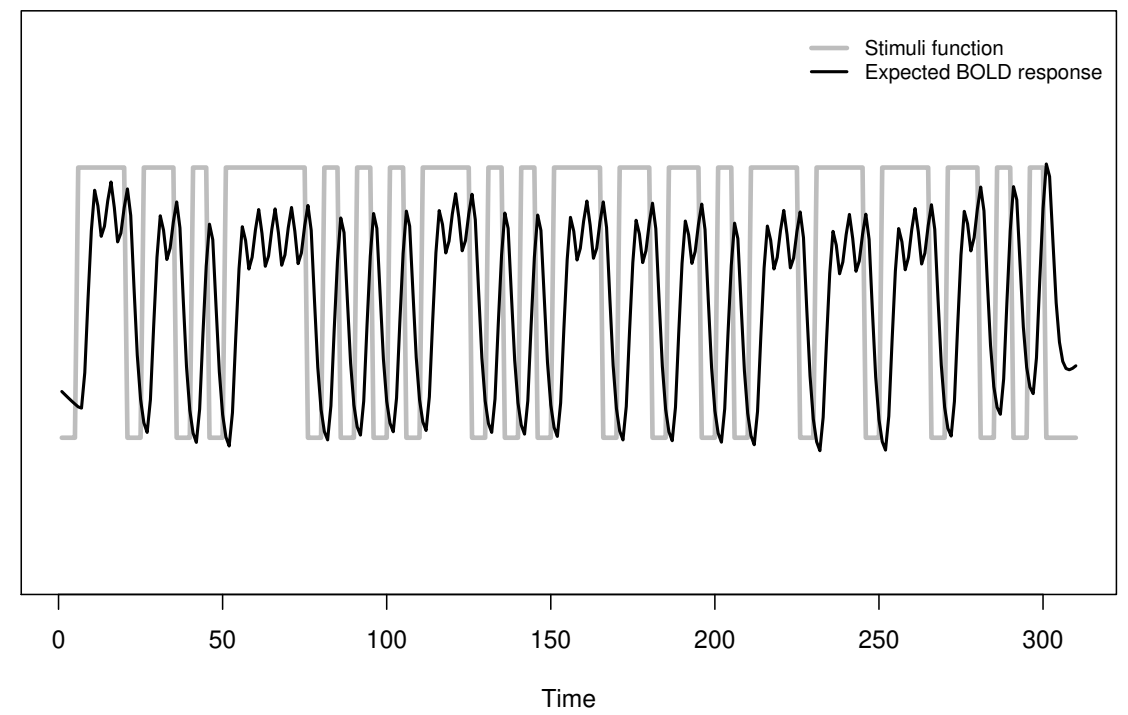

Figure 1: Merged stimuli function for voice and non-voice sounds with its respective expected BOLD response function for the "voice localizer" example.

In order to present a practical example using the method proposed in this work, we use the data from Pernet et al. [2015], which is openly available on OpenNeuro [Gorgolewski et al., 2017]. In that work, they perform an fMRI experiment where a group of $n=207$ individuals is submitted to sound stimulation composed by human voices and non-human sounds in order to identify or characterize the brain regions involved in the recognition of human voice sounds. For the sake of simplicity, we merge both human and non-human sounds in only one block design (figure 1) and take just 21 (sub-001:sub-021) from the 207 participants in the study. Our main aim with this example is to evaluate the capacity of our 
method to identify brain activation due to sound stimulation. It is also worth mentioning that brain images from all the subjects are converted to the MNI atlas [Brett et al., 2002] to make their brains comparable, which is a standard procedure in fMRI group analysis.

Marginal-FEST

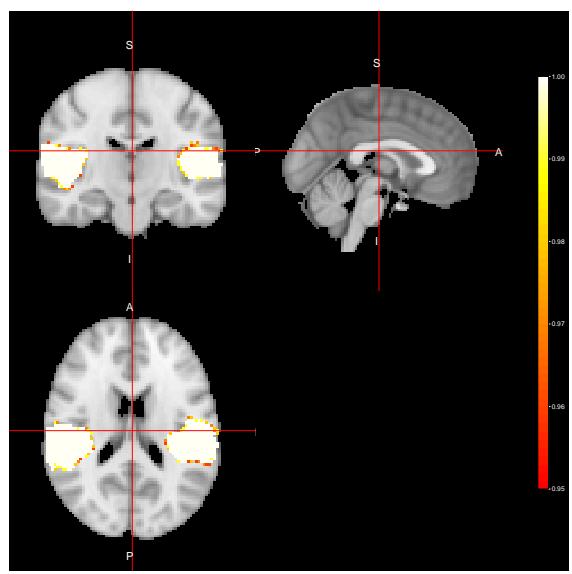

Joint-FEST

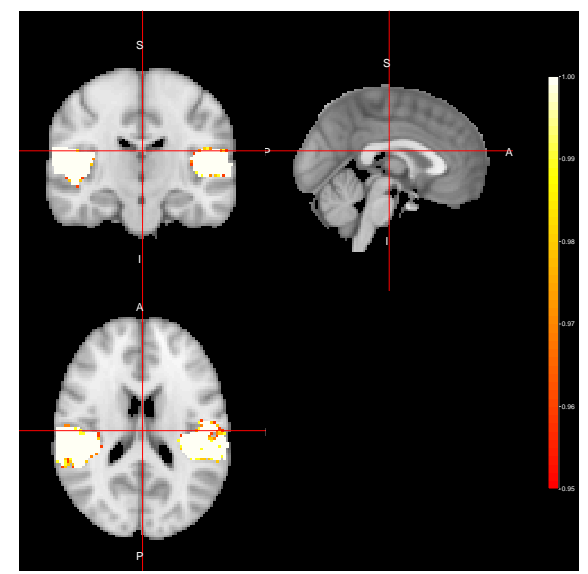

ACE-FEST

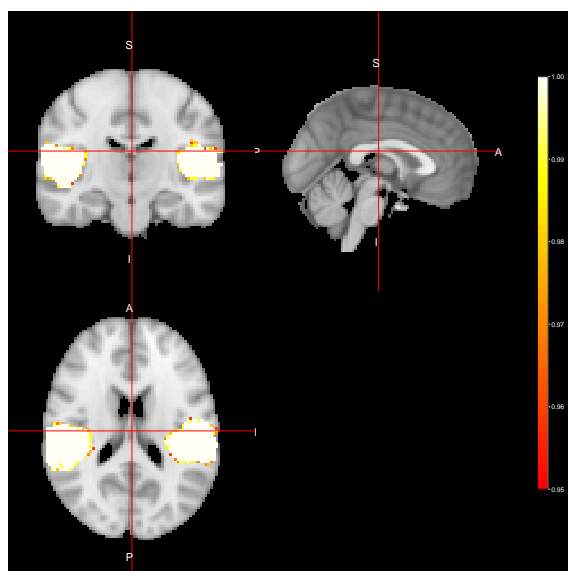

Figure 2: Activation Maps for the "voice localizer" example obtained when using the FEST algorithm under three different distributions (Marginal, Joint and LTT) related to the state parameter. 
Marginal-FFBS
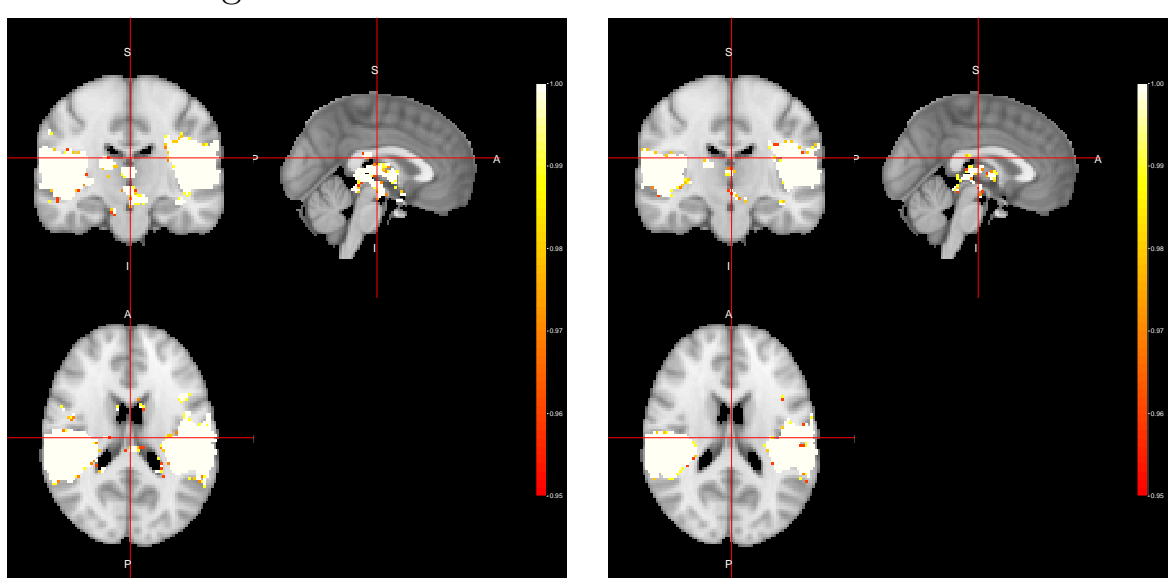

ACE-FFBS

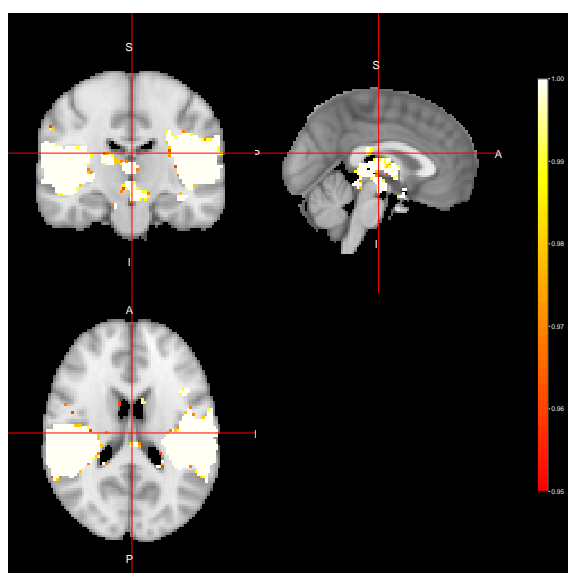

Figure 3: Activation Maps for the "voice localizer" example obtained when using the FFBS algorithm under three different distributions (Marginal, Joint and LTT) related to the state parameter.

From figures 2, 3 and 4, we can see the activation maps obtained for the "voice localizer" experiment using the method proposed in this work. From those images, we can say that the three algorithms (FEST, FFBS and FSTS) under the three different distributions (Marginal, joint and LTT or average distribution) successfully identify the temporal activation due to voice and non-voice sounds stimulation, nevertheless there are some slight differences among those maps worth mentioning. For instance, the maps obtained when using the FFBS algortihm allows for the identification of a broader activated region from the 
temporal cortex, however, on the other hand, it allows activations to appear (false-positive activations) on brain regions that should not be involved with this "voice localizer" experiment. On the other hand, more conservative results seem to be obtained when using FEST and FSTS algorithms, but with less false activations.
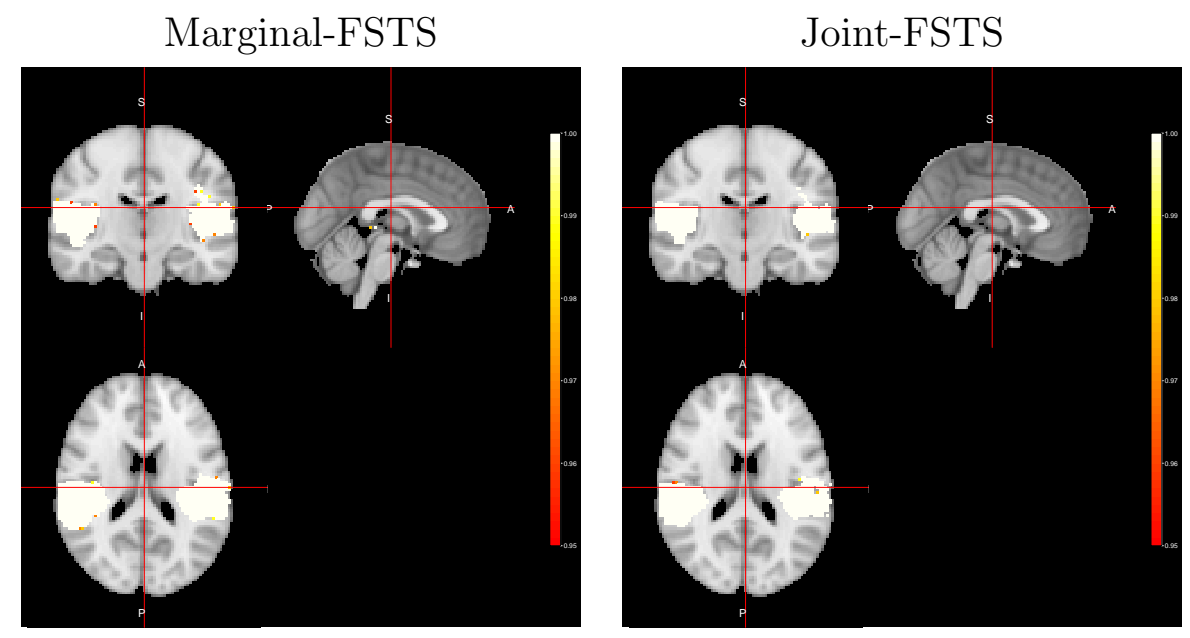

ACE-FSTS

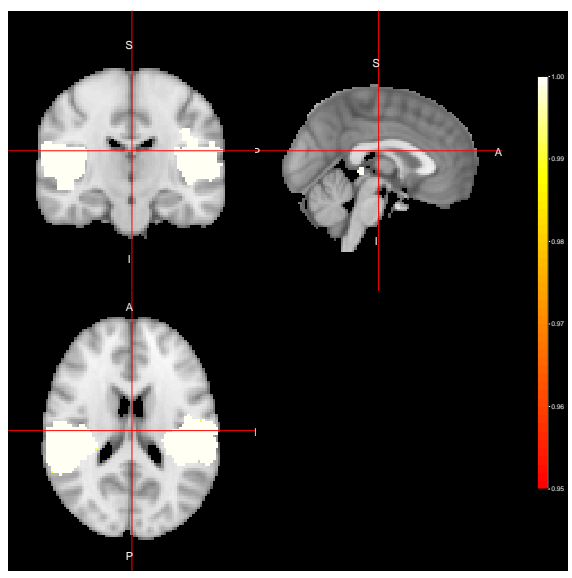

Figure 4: Activation Maps obtained for the "voice localizer" example when using the FSTS algorithm under three different distributions (Marginal, Joint and LTT) related to the state parameter. 
AG-algorithm(ACE-FEST)

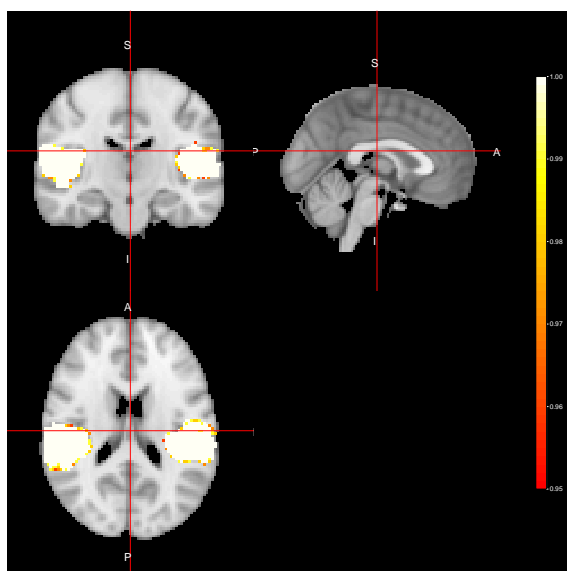

AG-algorithm(ACE-FSTS)

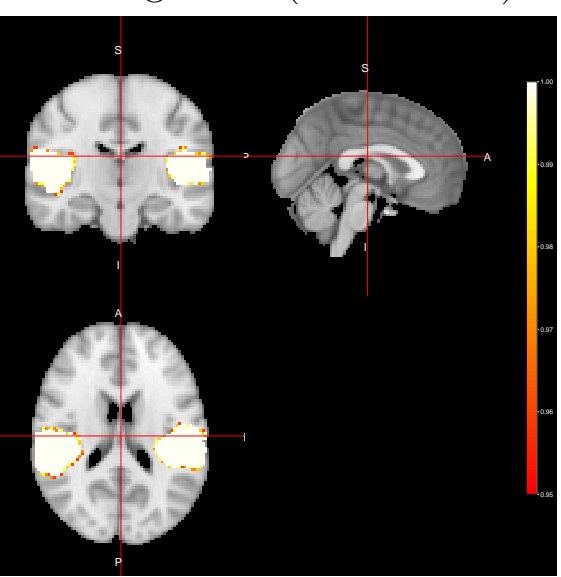

Figure 5: Activation Maps obtained for the "voice localizer" example when using the AGalgorithm

In figure 5, we can see group activation maps obtained when using the AG-algorithm for every sampler option (FEST, FFBS, FSTS) at the individual level. From this example, we can conclude that with the AG-algorithm it is also possible to identify brain activation when analysing fMRI data for group activation. 


\section{Concluding remarks}

In this work, we present a method for fMRI group analysis, which is just a continuation of our method [Jiménez et al., 2019] for fMRI data analysis using MDLM at the individual level. It has shown to be very effective when analyzing fMRI data for a group of 21 subjects from a "voice localizer" experiment. We also introduce a new algorithm (AG-algorithm), which allows us to sample on-line tractories of the state parameter from each subject individually, instead of combining the group information into a single posterior distribution (4) to sample from. What is intended with this approach is to allow more uncertainty from the intra-subject variability to be taken into account when assessing group voxel activation. Even though, no group comparison is performed in this paper, it can be easily implemented and we just let it as a future work. We also want to stress that other types of analysis, such as group analysis for repeated measures can be easily addressed under this setting thanks to the flexibility of the MDLM. Despite this method being successfully tested with many other fMRI data sets from different types of experiments, a more indepth assessment (as it is made in Jiménez et al. [2019]) using real and simulated data must be performed in order to offer a more reliable validation of it.

\section{References}

Johnatan Cardona Jiménez, Carlos A. de B. Pereira, and Victor Fossaluza. Assessing dynamic effects on a bayesian matrix-variate dynamic linear model: an application to fmri data analysis. arXiv:1910.12058, 2019.

Marcelo Camargo Batistuzzo, Joana Bisol Balardin, Maria da Graça Morais Martin, Marcelo Queiroz Hoexter, Elisa Teixeira Bernardes, Sonia Borcato, Marina de Marco e Souza, Cicero Nardini Querido, Rosa Magaly Morais, Pedro Gomes De Alvarenga, et al. Reduced prefrontal activation in pediatric patients with obsessive-compulsive disorder during verbal episodic memory encoding. Journal of the American Academy of Child $\&$ Adolescent Psychiatry, 54(10):849-858, 2015. 
Cyril R Pernet, Phil McAleer, Marianne Latinus, Krzysztof J Gorgolewski, Ian Charest, Patricia EG Bestelmeyer, Rebecca H Watson, David Fleming, Frances Crabbe, Mitchell Valdes-Sosa, et al. The human voice areas: Spatial organization and inter-individual variability in temporal and extra-temporal cortices. Neuroimage, 119:164-174, 2015.

Mark Jenkinson, Christian F Beckmann, Timothy EJ Behrens, Mark W Woolrich, and Stephen M Smith. Fsl. Neuroimage, 62(2):782-790, 2012.

William D Penny, Karl J Friston, John T Ashburner, Stefan J Kiebel, and Thomas E Nichols. Statistical parametric mapping: the analysis of functional brain images. Elsevier, 2011.

Christian F Beckmann, Mark Jenkinson, and Stephen M Smith. General multilevel linear modeling for group analysis in fmri. Neuroimage, 20(2):1052-1063, 2003.

Arjun K Gupta and Daya K Nagar. Matrix variate distributions. Chapman and Hall/CRC, 1999.

Krzysztof Gorgolewski, Oscar Esteban, Gunnar Schaefer, Brian Wandell, and Russell Poldrack. Openneuro - a free online platform for sharing and analysis of neuroimaging data. Organization for Human Brain Mapping. Vancouver, Canada, 1677, 2017.

Matthew Brett, Ingrid S Johnsrude, and Adrian M Owen. The problem of functional localization in the human brain. Nature reviews neuroscience, 3(3):243, 2002. 


\section{BayesDLMfMRI: Bayesian Matrix-Variate Dynamic Linear Models for fRMI modeling in $\mathbf{R}$}

Abstract This article introduces an $\mathrm{R}$ package to perform statistical analysis for task-based fMRI data at both individual and group levels. The analysis to detect brain activation at the individual level is based on modeling the fMRI signal using Matrix-Variate Dynamic Linear Models (MDLM). Therefore, the analysis for the group stage is based on posterior distributions of the state parameter obtained from the modeling at the individual level. In this way, this package offers several $\mathrm{R}$ functions with different algorithms to perform inference on the state parameter to assess brain activation for both individual and group stages. Those functions allow for parallel computation when the analysis is performed for the entire brain as well as analysis at specific voxels when it is required.

\section{Introduction: fMRI packages with options for Bayesian Analysis}

Statistical modeling of processed fMRI data is a challenging problem, which has caught the attention of the statistical community in the past two decades. Large amount of observations are usually obtained with only one subject in an fMRI session which makes the implementation of more appropriate and sophisticated statistical models that can account for the spatiotemporal structures that are usually present in this type of data really challenging. From a Bayesian perspective, there have been published and proposed different types of models to model fMRI data (see for instance Zhang et al. (2016), Eklund et al. (2017), Bezener et al. (2018), Yu et al. (2018) and a complete review by Zhang et al. (2015)). Despite that, just a few software implementations of Bayesian methods are available for fMRI data analysis. For instance, the FSL software (Jenkinson et al., 2012) offers a Bayesian procedure for the group stage analysis, which depends on a Frequentist or Classical output from the individual stage. Another popular software among practioners in the neurosicience community with implementations of Bayesian methods is the SPM package (Penny et al., 2011). It has alternatives for Bayesian analysis to both individual and group stages, but as in the case of the FSL package, the group analysis depends on a Frequentist individaul stage output. There is also available a MATLAB GUI called NPBayes-fMRI (Kook et al., 2019), which is an implementation of the work proposed by Zhang et al. (2016), a fully Bayesian modeling for individual and group stages. In R (R Core Team, 2018), tools for different types of fMRI data analysis are provided in packages like fmri (Tabelow and Polzehl, 2011), oro.nifti (Whitcher et al., 2011), neurobase (Muschelli, 2018), and neuRosim (Welvaert et al., 2011). For fMRI data analysis under a Bayesian approach there was a package called cudaBayesreg (da Silva et al., 2011), which was removed recently from the CRAN repository. Thus, the package BHMSMAfMRI (Sanyal and Ferreira, 2019) is to our knowledge the only Bayesian option for fMRI data analysis in R to this date.

In this work, we are presenting an R package called BayesDLMfMRI, which is an implementation of the method proposed in Jiménez et al. (2019) and Jiménez (2019). The BayesDLMfMRI permits to perform fMRI individual and group analysis based on the Matrix-Variate Dynamic Linear Model (MVDLM) proposed by Quintana (1985). Given this type of analysis usually involved large amounts of data, we take advantage of the packages Rcpp (Eddelbuettel et al., 2011) and RcppArmadillo (Eddelbuettel and Sanderson, 2014) in order to speed up the computation time. BayesDLMfMRI also depends internally on the package pbapply (Solymos and Zawadzki, 2019), which allows the user to visualize a progress bar while the process is run in either sequence or parallel. In order to run an analysis using our package the user must provide a $4 \mathrm{D}$ array (or a $4 \mathrm{D} \times N$ array for a group analysis with $N$ subjects) containing the sequence of processed images and a design matrix whose columns are related to the so-called expected blood-oxygen-Level dependent (BOLD) response and/or some other covariates related to particular subjects' characteristics. To process the raw images, we recommend the use of packages such as FSL or SPM. And to build the expected BOLD response the user has options like the fmri package, which allows defining different types of models to represent the haemodynamic response function (HRF). BayesDLMfMRI package is intended to be just another well tested and assessed tool for the practitioners who are interested to perform statistical analysis of processed fMRI images from a Bayesian perspective. In the next section, we give a brief description of the model and methods on which the BayesDLMfMRI package is based and present its R functions to perform fMRI data analysis. In section three, we present some examples to illustrate the use of the package and in the final section, we give some concluding remarks. 


\section{Methods and software}

\begin{tabular}{|c|c|}
\hline Function & Description \\
\hline (ffdGroupEvidenceFEST) ffdEvidenceFEST & $\begin{array}{l}\text { It returns 3D arrays to build (group) indi- } \\
\text { vidual activation evidence maps (based } \\
\text { on outputs from MVDLM) fitting an } \\
\text { MVDLM at individual level and using the } \\
\text { FEST algorithm to assess voxel activation. } \\
\text { There are two options related to the pos- } \\
\text { terior distribution of } \Theta_{[(i, j) k t}^{(z)}: \text { LLT and Joint. } \\
\text { Each one runs independently and must be } \\
\text { set by the user as an input parameter. }\end{array}$ \\
\hline (ffdGroupEvidenceFFBS) ffdEvidenceFFBS & $\begin{array}{l}\text { It returns 3D arrays to build (group) indi- } \\
\text { vidual activation evidence maps (based } \\
\text { on outputs from MVDLM) fitting an } \\
\text { MVDLM and using the FFBS algorithm to } \\
\text { assess voxel activation. The options LLT } \\
\text { and Joint related to the posterior distribu- } \\
\text { tion of } \Theta_{[i, j, k] t}^{(z)} \text { are simultaneously executed } \\
\text { in the same run. }\end{array}$ \\
\hline (ffdGroupEvidenceFSTS) ffdEvidenceFSTS & $\begin{array}{l}\text { Same features as (ffdGroupEvidenceFFBS) } \\
\text { ffdEvidenceFFBS, though using the FSTS } \\
\text { algorithm. }\end{array}$ \\
\hline (GroupSingleVoxelFEST) SingleVoxelFEST & $\begin{array}{l}\text { Produces some usefull outputs from a sin- } \\
\text { gle voxel analysis related to the FEST al- } \\
\text { gorithm }\end{array}$ \\
\hline (GroupSingleVoxelFFBS) SingleVoxelFFBS & $\begin{array}{l}\text { Same features as (GroupSingleVoxelFEST) } \\
\text { SingleVoxelFEST, though using the FFBS } \\
\text { algorithm }\end{array}$ \\
\hline (GroupSingleVoxelFSTS) SingleVoxelFSTS & $\begin{array}{l}\text { Same features as (GroupSingleVoxelFEST) } \\
\text { SingleVoxelFEST, though using the FSTS } \\
\text { algorithm }\end{array}$ \\
\hline
\end{tabular}

Table 1: Functions implemented in the BayesDLMfMRI package for fMRI (group) individual analysis. LLT stands for Transformation Linear Test, which is the same as the average cluster effect (ACE) distribution defined in ArchiveRef.

The type of MVDLM which this package relies on is the version originally developed by Quintana (1985) and Quintana (1987). Here, we just give a brief description of the model and methods. For a better understanding of the method implemented in this package for the individual stage, see Jiménez et al. (2019). Let $\mathbf{Y}_{[i, j, k] t}^{(z)}$ be a $q \times 1$ random vector representing the cluster of observed BOLD responses at position $(i, j, k)$ in the brain image, time $t$ and subject $z$, for $i=1, \ldots, d_{1}, j=1, \ldots, d_{2}, k=1, \ldots, d_{3}$, $t=1, \ldots, T$ and $z=1 \ldots, N$. Thus, the cluster of BOLD signals is modeled as

$$
\begin{aligned}
& \text { Observation: } \quad \mathbf{Y}_{[i, j, k \mid t}^{(z)}=\mathbf{F}_{t}^{\prime} \Theta_{[i, j, k t}^{(z)}+\boldsymbol{v}_{(i, j, k \mid t}^{\prime(z)} \\
& \text { Evolution: } \quad \Theta_{[i, j, k\}}^{(z)}=\mathbf{G}_{t} \Theta_{[i, j, j] t-1}^{(z)}+\Omega_{[i, j, k\} t}^{(z)},
\end{aligned}
$$

where, for each $t$ we have a $q \times 1$ vector $\boldsymbol{v}_{[i, j, k \mid t}^{\prime(z)}$ of observational errors, a $p \times q$ matrix $\Theta_{[i, j, k \mid t}$ of state parameters, a $p \times q$ matrix $\Omega_{[i, j, k \mid t}^{(z)}$ of evolution errors. The $1 \times p$ and $p \times p$ matrices $\mathbf{F}_{t}^{\prime}$ and $\mathbf{G}_{t}$ respectivelly are common to each of the $q$ univariate DLMs. The covariates related to the design being used, either a block or an event-related design as well as other characteristics of the subjects, can be included in the columns of $\mathbf{F}_{t}^{\prime}$. For individual analysis the model (1) is fitted to every cluster of voxels related to position $[i, j, k]$ in the brain image and the cluster size depends on the $r$ distance, which is a parameter defined by the user. To identify wether or not there is significant evidence of cluster activation or brain reaction at region $[i, j, k]$, three different algorithms (FEST, FSTS and FFBS) proposed in Jiménez et al. (2019) are implemented. Those algorithms are sampling schemes that allow to draw on-line trajectory curves related to the state parameter $\Theta_{[i, j|k| t}^{(z)}$, and with those resulting samples a Monte Carlo evidence related to the event of cluster activation is computed. The information obtained from the first stage can be combined in different ways to produce several measures of evidence for the 
group activation. For a better understanding of the method implemented in this package for the group stage, see Jiménez (2019). In table 1, the package's functions for (group) individual analysis and a brief description of them are presented.

\section{Illustrations}

In order to show some practical illustrations about the use of the BayesDLMfMRI package, we use data related to an fMRI experiment where a sound stimilus is presented. That experiment is intended to offer a "voice localizer" scan, which allows rapid and reliable localization of the voice-sensitive "temporal voice areas" (TVA) of human auditory cortex (Pernet et al., 2015). The data of this "voice localizer" scan is freely available on the online platform OpenNEURO (Gorgolewski et al., 2017). In the original experiment, the voice and non-voice sounds are separately analyzed, but here we merge both sounds in one block as it were just one stimulus (see figure 1). For the individual analysis, we select one from the 217 subjects whose data are available on OpenNEURO, specifically, we take the data from sub-007. To illustrate the use of the group analysis functions, we take 20 subjects (sub-001:sub-021). The raw fMRI data is preprocessed using the standard processing pipelines implemented on the FSL software for motion correction, spatial smoothing and other necessary procedures to perform statistical analysis for both individual and group stages.

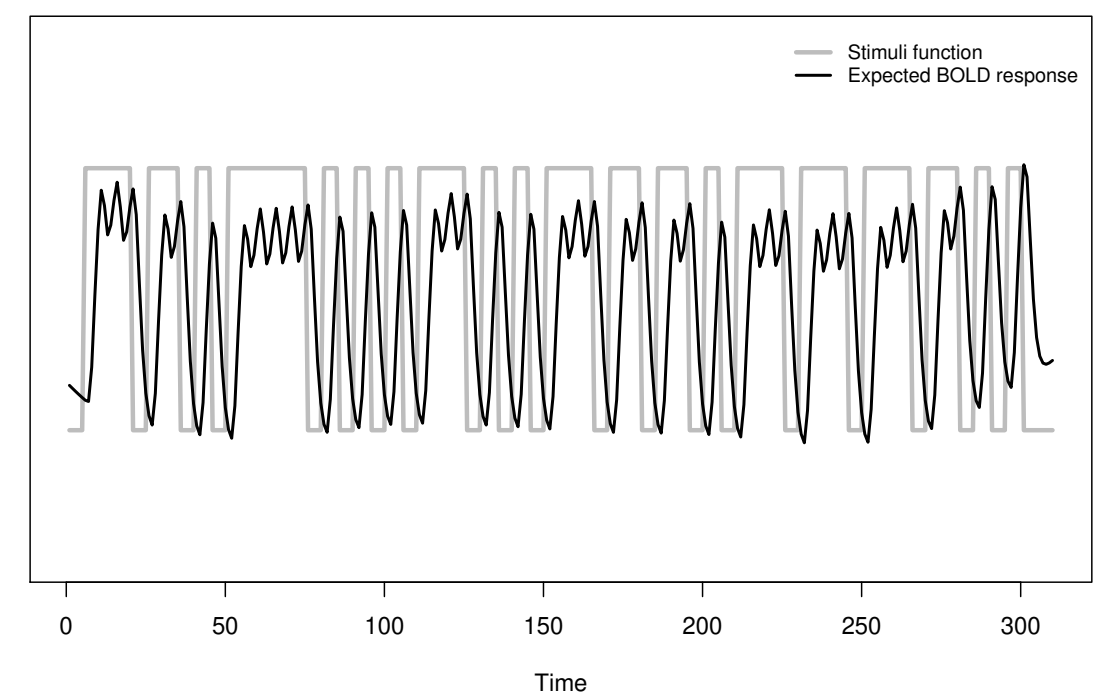

Figure 1: Merged stimuli function for voice and non-voice sounds with its respective expected BOLD response function for the "voice localizer" example.

\section{Individual analysis}

In order to run any of the functions related to individual analysis, the user must provide two inputs: an array of four dimensions containing the MRI images and a matrix whose columns contain the covariates to model the observed BOLD response. Thus, we read the data.nii.qz file, which contains the MRI images, using the function readNIFTI from the package oro.nifti and the covariates.cvs file which contains the expected BOLD response (show in figure 1) and its derivative respectively.

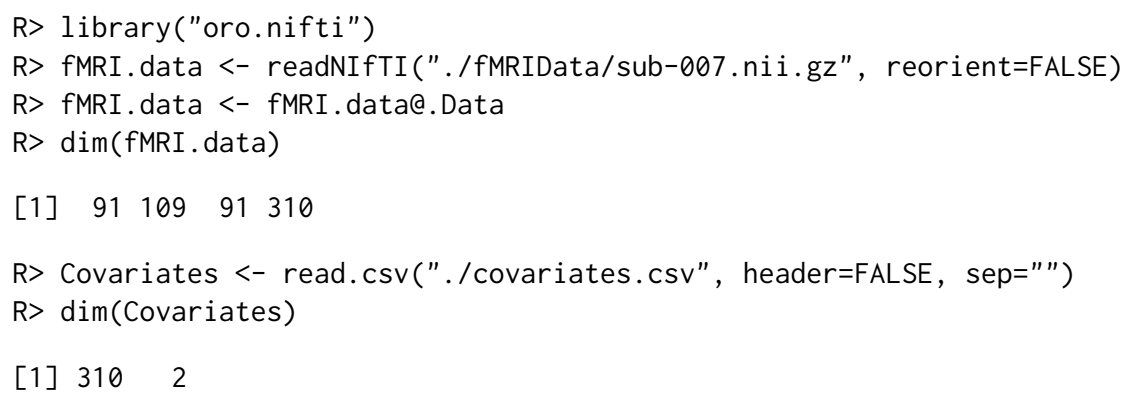


To perform a voxel-wise anaylsis ${ }^{1}$ to obtain a 3D array with measurements of activation evidence for every voxel, the user has three options (ffdEvidenceFEST, ffdEvidenceFFBS and ffdEvidenceFSTS), which at the same time can yield three different types of evidence measurements for voxel activation. Just to ilustrate their use, we run them using the "voice localizer" example.

R> install.packages("BayesDLMfMRI_1.0.tar.gz", repos=NULL)

R> library (BayesDLMfMRI)

$\mathrm{R}>$ res <- ffdEvidenceFEST $(\mathrm{ffdc}=\mathrm{fMRI}$. data, covariates = Covariates,

$+\mathrm{m} \theta=0$, Cova $=100$, delta $=0.95, \mathrm{~s} 0=1, \mathrm{n} \theta=1, \mathrm{Nsimu} 1=100$, Cutpos $1=30$,

$+r 1=1$, Test $=$ "LTT", Ncores $=15$ )

$|++++++++++++++++++++++++++++++++++++++++++++++++++| 100 \%$ elapsed $=05 \mathrm{~m} 36 \mathrm{~s}$

The arguments $\mathrm{m} \theta, \mathrm{Cova}, \mathrm{s} \theta$ and $\mathrm{n} \theta$ are the hyper-parameters related to the joint prior distribution of $\left(\Theta_{\mid i, i k t, t}^{(z)}, \Sigma_{(i, j, k t}^{(z)}\right)$. For this example, we are setting a "vague" prior distribution acording to Quintana and West (1987), where $m \theta=0$ define a null matrix with zero values in all its entries and both Cova $=100$ and $\mathrm{SO}=1$ define diagonal matrices. $r 1$ is the euclidean distance, which defines the size of the cluster of voxels jointly modeled. Test is the parameter related to the test selected by the user, for which there are two options: "LTT" and "Joint". "Ncores" is the argument related to the number of cores when the process is executed in parallel. Nsimu1 is the number of simulated on-line trajectories related to the state parameter $\Theta_{|i, j, k| t}^{(2)}$. From our own experience dealing with different sets of fMRI data, we recommend Nsimu1 $=100$ as a good number of draws to obtain reliable results. Cutpos 1 is the time up from where the on-line trajectories are considered in order to compute the activation evidence and delta is the value of the discount factor. For a better understanding about the setting of these two last arguments, see (Archive1).

$\mathrm{R}>\operatorname{str}($ res $)$

List of 2

$\$:$ num $[1: 91,1: 109,1: 91] 00000000000$.

$\$:$ num $[1: 91,1: 109,1: 91] 0000000000 \ldots$

$R>\operatorname{dim}(\operatorname{res}[[1]])$

[1] $91 \quad 109 \quad 91$

The output for the ffdEvidenceFEST function depends on the type of Test set by the user. For Test $=$ "LTT" the function returns a list of the type $\operatorname{res}[[p]][x, y, z]$, where $[[p]]$ represents the column position in the covariates matrix and $[x, y, z]$ represent the voxel position in the brain image. Thus, for the "voice localizer" example res[[1]] and res[[2]] are the 3D arrays related to the evidence for brain activation related to the BOLD response for the auditory stimuli and its derivative respectively. When Test $=$ Joint the output returned is an array

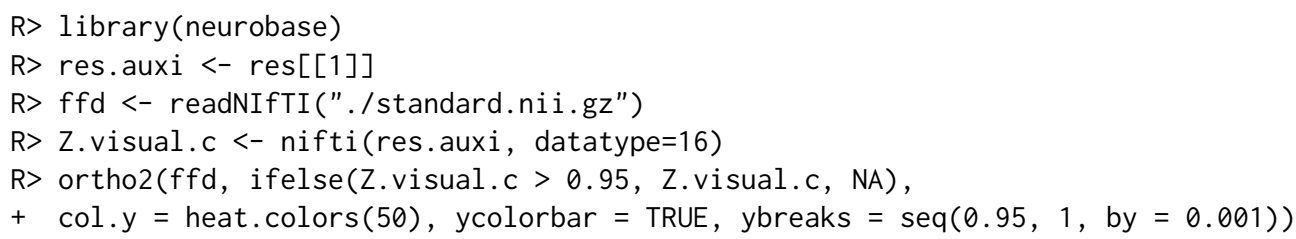

The neurobase package is one amongst several options available in $\mathrm{R}$ to visualize MRI images. In this example, we use its ortho2() function in order to plot the evidence activation map. The standar.nii.gz contains the MNI brain atlas, which is used in this work as a reference space for individual and group analysis. For a better understanding of the use of brain atlas, see Brett et al. (2002).

\footnotetext{
${ }^{1}$ The BayesDLMfMRI package depends on Rcpp, RcppArmadillo, RcppDist and pbapply packages.
} 

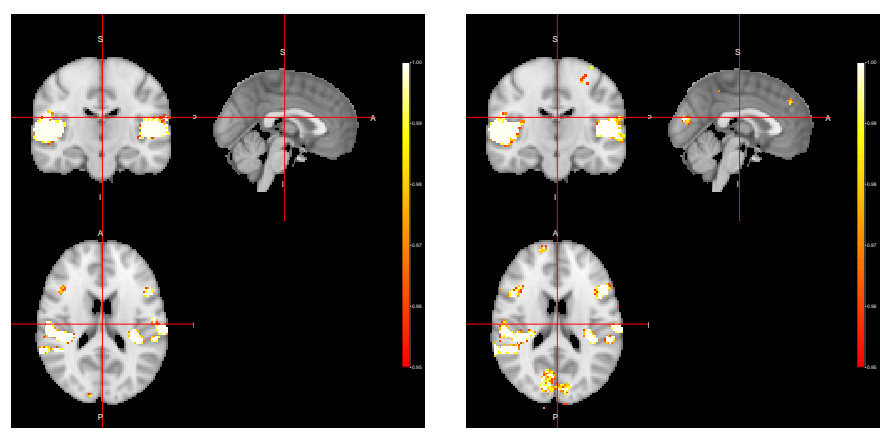

Figure 2: Activations maps obtained with the functions ffdEvidenceFEST (left) and ffdEvidenceFFBS (right) using the "Joint" as sampler distribution.

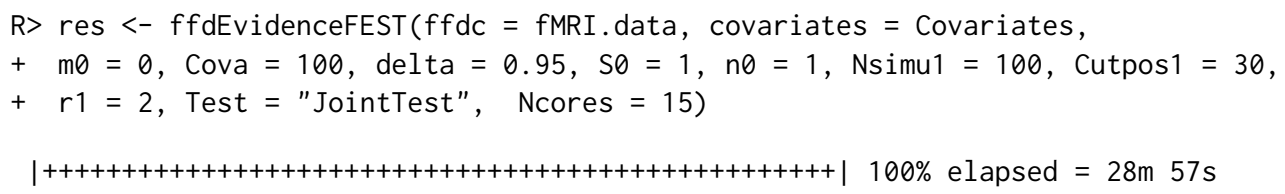

$\mathrm{R}>\operatorname{str}($ res $)$

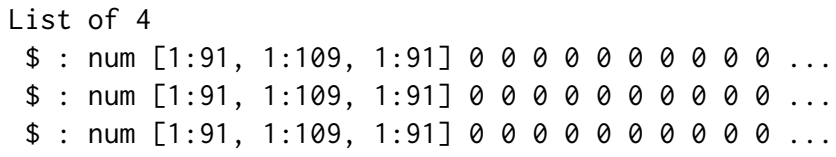

For both $f f d E v i d e n c e F F B S$ and $f f d E v i d e n c e F S T S$ the input arguments and output structures are the same. Below we run the "voice localizer" example using the ffdEvidenceFFBS function. It returns a list of the form $\operatorname{res}[[T]][p, x, y, z]$, where $T$ defines the type of test $(T=1$ for "Marginal", $T=2$ for "JointTest", and $T=3$ for "LTT"), $p$ represents the column position in the covariates matrix and $x, y, z$ represent the voxel position in the brain image.

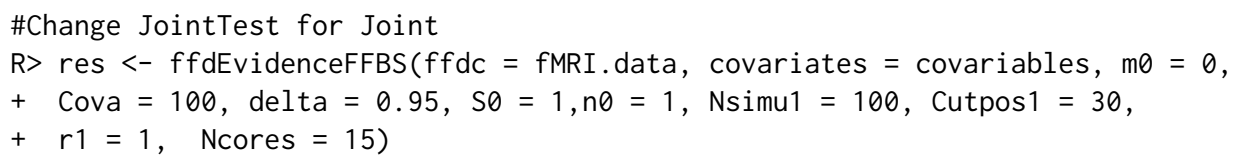

The BayesDLMfMRI package also has functions that allow taking a closer look for specific voxels defined by the user. For instance, let's suppose we are interested to see some of the output elements related to the FEST algorithm for an active voxel when using the LTT test.

R> \#Identifying active voxels for a probability threshold of 0.99

$\mathrm{R}>$ active.voxels $=$ which $(\operatorname{res}[[1]]>0.99$, arr.ind $=$ TRUE)

$\mathrm{R}>$ head(active.voxels) 


$\begin{array}{lrrr} & \operatorname{dim} 1 & \operatorname{dim} 2 & \operatorname{dim} 3 \\ {[1,]} & 28 & 75 & 22 \\ {[2,]} & 22 & 71 & 24 \\ {[3,]} & 22 & 72 & 24 \\ {[4,]} & 23 & 72 & 24 \\ {[5,]} & 23 & 73 & 24 \\ {[6,]} & 24 & 73 & 24\end{array}$

$\mathrm{R}>\mathrm{N} 1<-\operatorname{dim}($ covariables) [1]

R> res.indi <- SingleVoxelFEST(posi.ffd $=c(14,56,40)$, covariates

$+=$ Covariates, $\mathrm{ffdc}=\mathrm{fMRI}$. data, $\mathrm{m} \theta=0$, Cova $=100$, delta $=0.95, \mathrm{~s} 0=1$,

$+\mathrm{n} 0=1, \mathrm{Nsimu} 1=100, \mathrm{~N} 1=\mathrm{N} 1$, Cutpos1 $=30$, Min.vol $=0.10, \mathrm{r} 1=1$,

+ Test $=$ "LTT")

R> $\operatorname{str}($ res.indi)

List of 4

$\$$ Eviden : num $[1,1: 2] 0.980$

\$ Online_theta: num $\left[\begin{array}{llllllll}1: 280, & 1: 2,1: 100] & 2.57 & 2.52 & 2.54 & 2.62 & 2.46 & \ldots\end{array}\right.$

$\$$ Y_simu : num $[1: 280,1: 100] 1.7850 .5210 .624 \quad 1.028 \quad-0.165 \ldots$

$\$$ FitnessV : num 0.762

The function IndividualVoxelFEST() requires just a few additional input arguments: the position of the voxel in the brain image (posi.ffd), the last period of time of the temporal series $\left(\mathbf{Y}_{[i, j, k] t=1}\right.$ : $\mathbf{Y}_{[i, j, k \mid t=N 1}$ ) that are used in the analysis (N1) and Min.vol, which helps to define a threshold for the voxels that are considered in the analysis. For example, Min. vol $=0.10$ means that all the voxels with values below to $\max (\mathrm{fMRI}$. data) * Min.vol are discarted from the analysis. The output is a list containing a vector (Eviden) with the evidence measure of activation for each one of the $p$ covariates considered in the model, the simulated online trajectories of $\Theta_{|i, j, k| t}^{(z)}$ (Online_theta), the simulated BOLD responses (Y_simu) and measure to examine the goodnes of fit of the model $\left(100 *\left|\mathbf{Y}_{[i, j, k] t}-\hat{\mathbf{Y}}_{[i, j, k] t}\right| / \hat{\mathbf{Y}}_{[i, j, k] t}\right)$ for that particular voxel (FitnessV).

R> res.indi2 <- singleVoxelFEST(posi.ffd $=c(14,56,40)$,

+ covariates $=$ covariates, $f f d c=$ fMRI. data, $m \theta=0$, Cova $=100$,

+ delta $=0.95, \mathrm{~S} 0=1, \mathrm{n} 0=1, \mathrm{Nsimu} 1=100, \mathrm{~N} 1=\mathrm{N} 1$, Cutpos $1=30$, Min. vol $=0.10$,

$+r 1=1$, Test $=$ "JointTest")

R> $\operatorname{str}($ res.indi2)

List of 5

\$ EvidenMultivariate: num $[1,1: 2] 0.810$

\$EvidenMarginal : num [1, 1:2] 0.980

\$Online_theta : num $[1: 2,1: 280,1: 100] 2.38-1.72 .42-1.772 .35 \ldots$

\$Y_simu : num $[1: 100,1: 7,1: 280] \quad 0.597 \quad 0.883 \quad 1.7160 .110 .344 \ldots$

$\$$ FitnessV : num $0.805 \$$

When Test $=$ "Joint", the function IndividualVoxelFEST returns two measures of voxel activation related to the joint and marginal tests along with the already explained remain list elements.

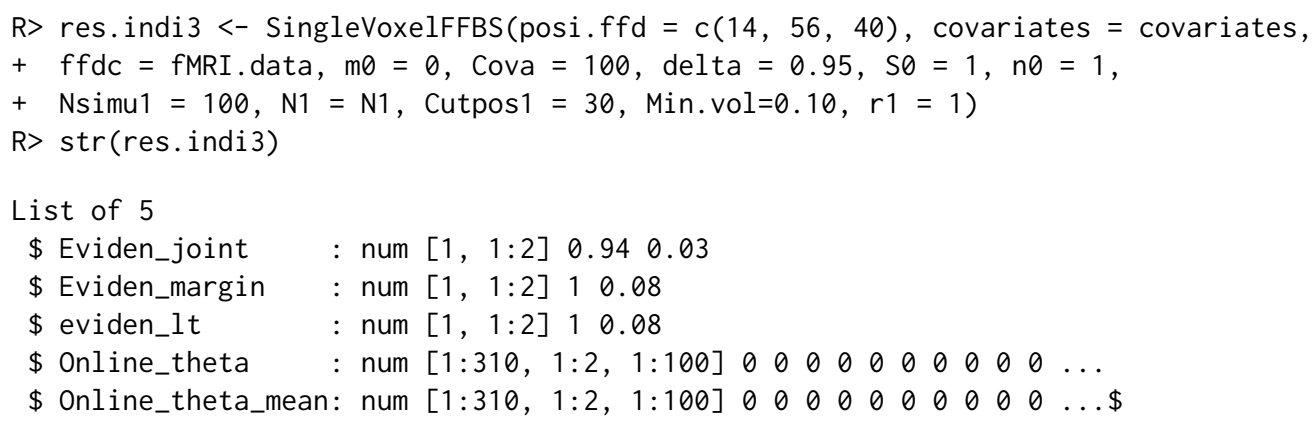

For both functions IndividualVoxelFFBS and IndividualVoxelFSTS the input arguments and output estructures are exactly the same. Three measures of evidence related to the joint, marginal and linear transformation (LTT) tests are generated in the same run. There are also two elements from the list output related to the online simulated trajectories of the state parameter. Online_theta are the draws for the marginal model and Online_theta_mean are the mean draws obtained from the joint model. 
$R>$ frame ()

$\mathrm{R}>$ plot. $w$ indow $(x \lim =\mathrm{c}(30,311), \mathrm{ylim}=\mathrm{c}(-5,5))$

$\operatorname{axis}(1, \operatorname{at}=\operatorname{seq}(30,310$, by $=20), \quad l w d=2, x l a b=$ "Time")

$\mathrm{R}>$ box $(1 w d=2)$

$R>$ for (i in 1:dim(res.indi\$Y_simu)[2])\{lines(31:dim(covariates)[1],

+ res.indi\$Y_simu[, i])\}

R> lines(31:dim(covariates)[1], covariates[31:dim(covariates)[1], 1],

$+\operatorname{col}=$ "red", lwd = 2)

$\mathrm{R}>$ frame ()

R> plot.window $(x \lim =c(30,311), y \lim =c(-0.5,4.5), y l a b=$ expression(theta))

$\mathrm{R}>\operatorname{axis}(1, \operatorname{at}=\operatorname{seq}(30,310$, by $=20), \operatorname{lwd}=2, x \operatorname{lab}=$ "Time")

$R>\operatorname{axis}(2$, at $=-1: 4, \quad 1 w d=2)$

$\mathrm{R}>\operatorname{box}(1 w d=2)$

$\mathrm{R}>$ for ( $\mathrm{i}$ in 1:dim(res.indi $\$ 0$ nline_theta)[2])\{lines(31:dim(covariates)[1],

+ res.indi\$Online_theta[, i])\}

$\mathrm{R}>$ abline $(\mathrm{h}=0, \mathrm{col}=$ "red",$l w d=2)$

posi.ffd $=c(14,56,40)$

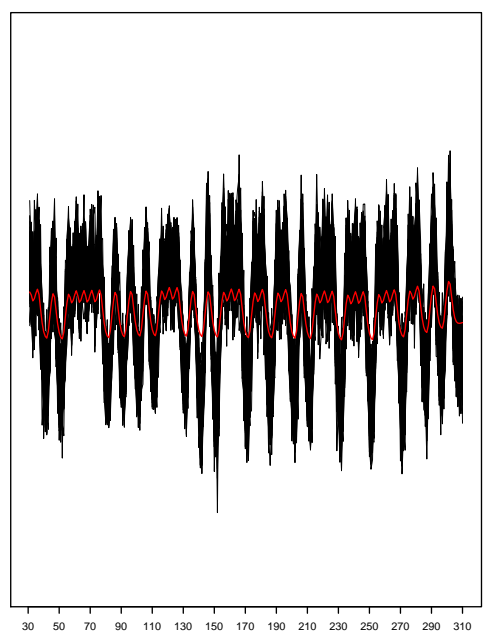

posi.ffd $=c(28,67,15)$

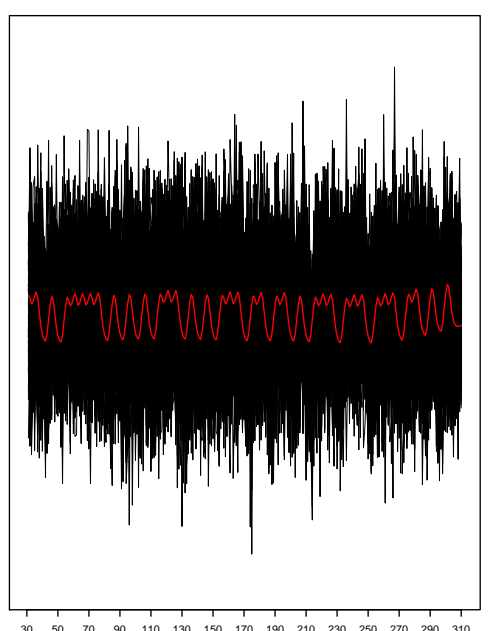

Figure 3: Simulated Bold response (black lines) and expected Bold response (red line) for an active (left) and non-active (right) voxel respectively. 
posi.ffd $=c(14,56,40)$

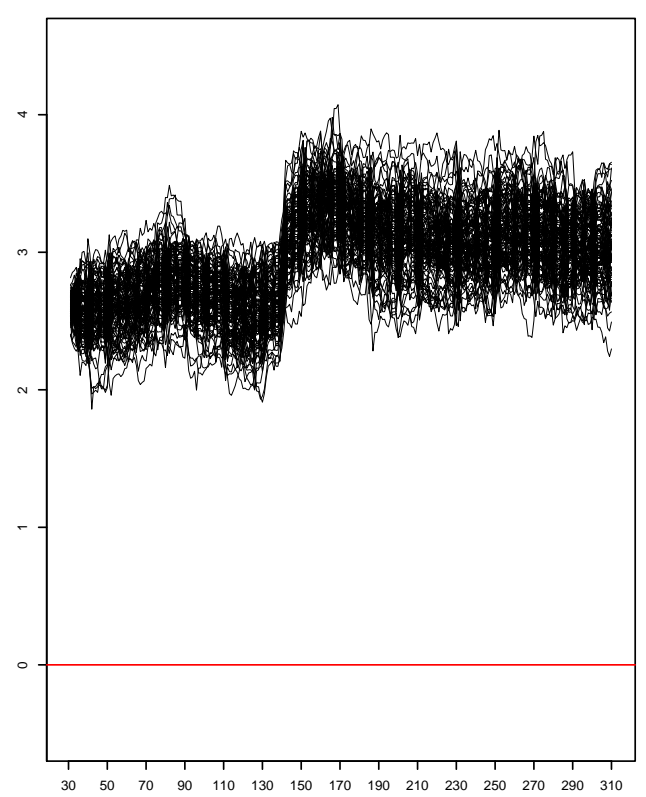

posi.ffd $=c(28,67,15)$

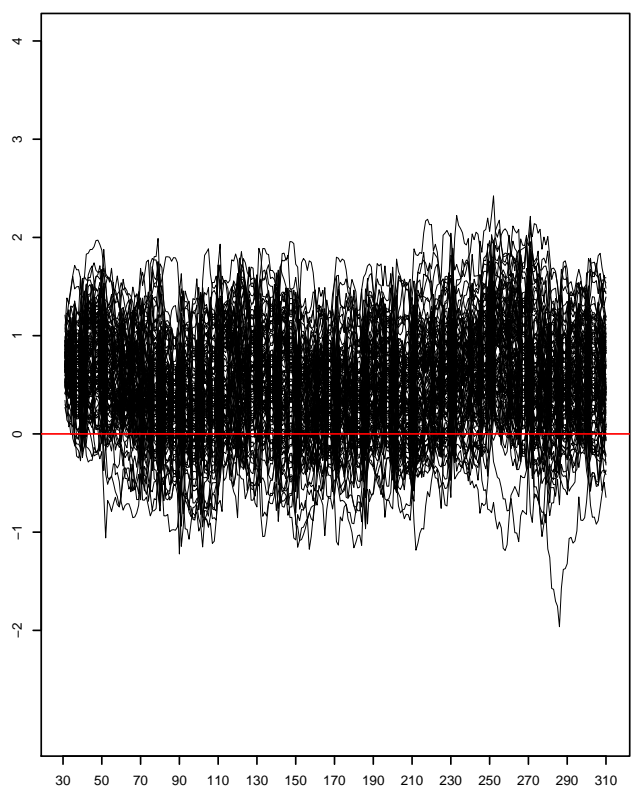

Figure 4: Simulated online trajectories of the state parameter for an active (left) and nonactive (right) volxel respectively.

\section{Group analysis}

Now we ilustrate how to run an fMRI group analysis as it is described in (RefARchive2). First, we read the fMRI images of 21 subjects from the "voice-localizer" example:

R> names <- list.files(" /fMRIData/")

$\mathrm{R}>$ names

using the "Joint" as sampler distribution.

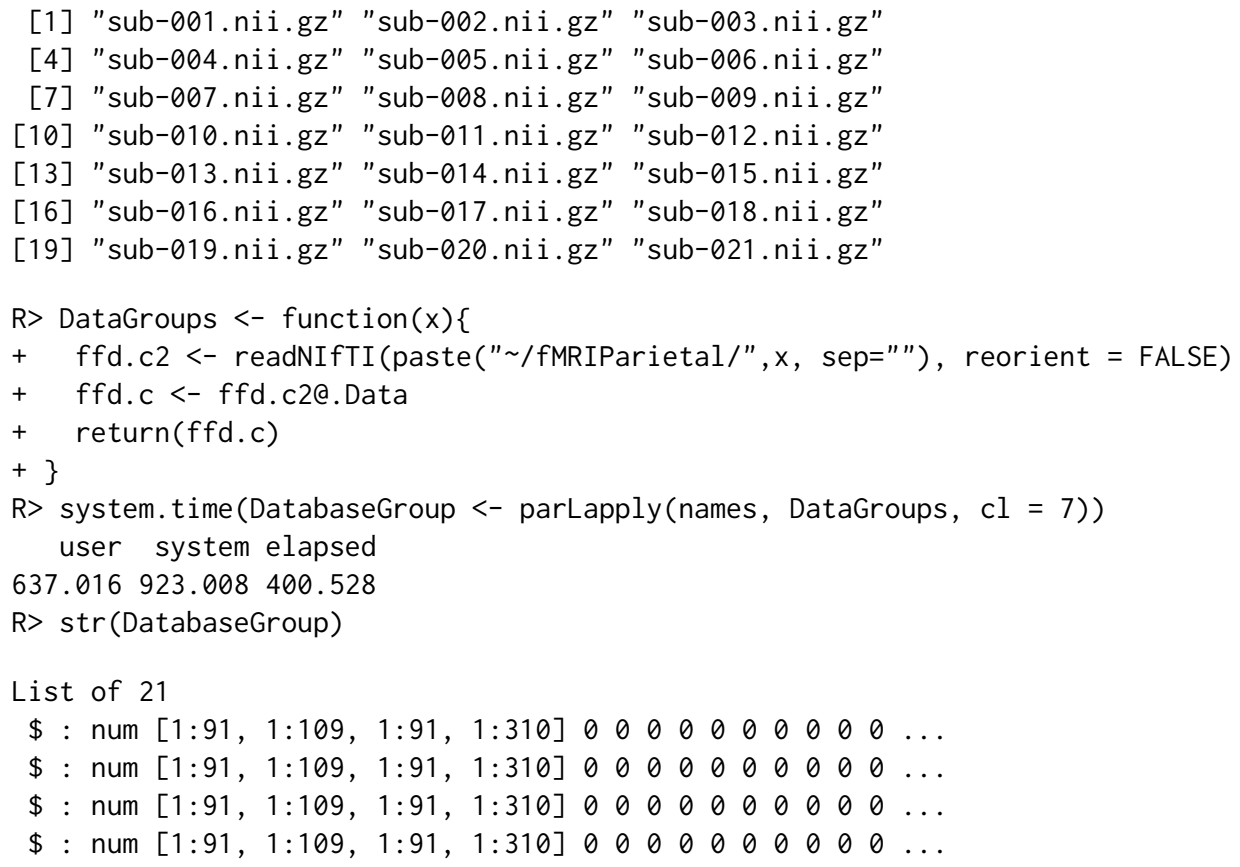




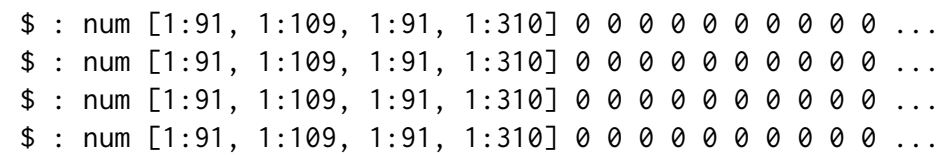

In order to run any of the functions available in this package to perform fMRI group analysis, the datasets or set of images from each subject must be stored on a list object as it is shown above. To deal with this huge amount of information the user has to have a big RAM memory capacity available on the machine where this process is going to be run. It is also recommended to have a multi-core processor available in order to speed up computation time. The arguments or input parameters for any of the functions offered in this package to run group analysis are almost the same as those required for individual analysis. There is only an additional argument needed (mask), which adds a 3D array that works as a brain of reference (MNI atlas) for the group analysis.

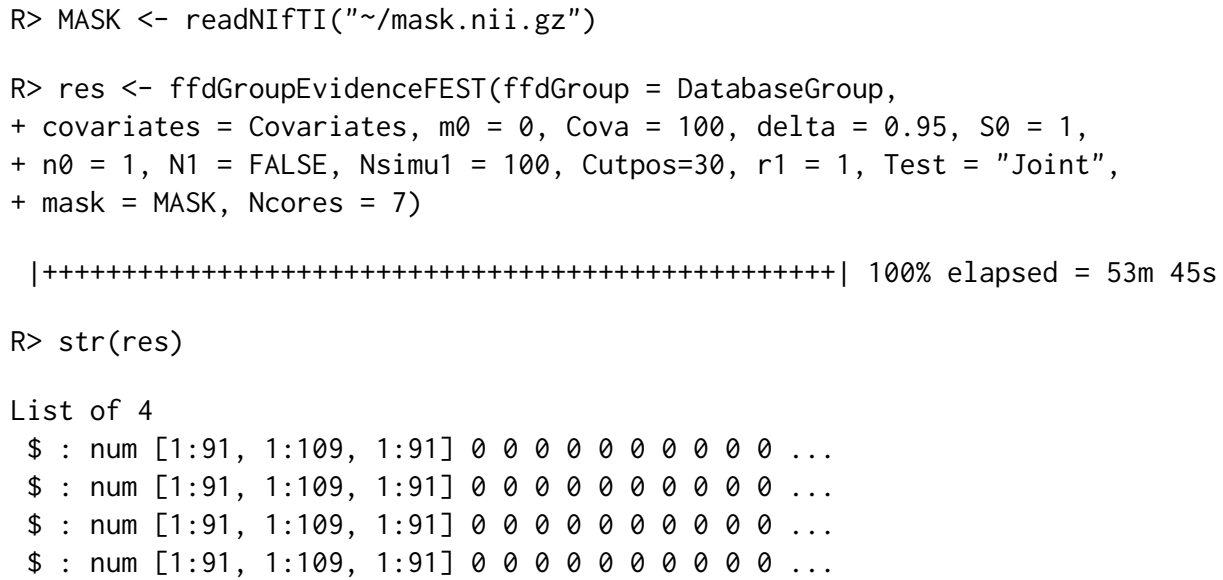

ffdGroupEvidenceFEST returns an array of dimension $2 \times p$ elements, where $p$ is the number of covariates and 2 is the number of options evaluated as sampler distributions: "Joint" and "Marginal". The first $p$ elements are the 3D arrays related to each column of the covariates matrix respectively when computing the activation evidence using the "Join" distribution. The ramaining arrays are those related to the marginal distribution.

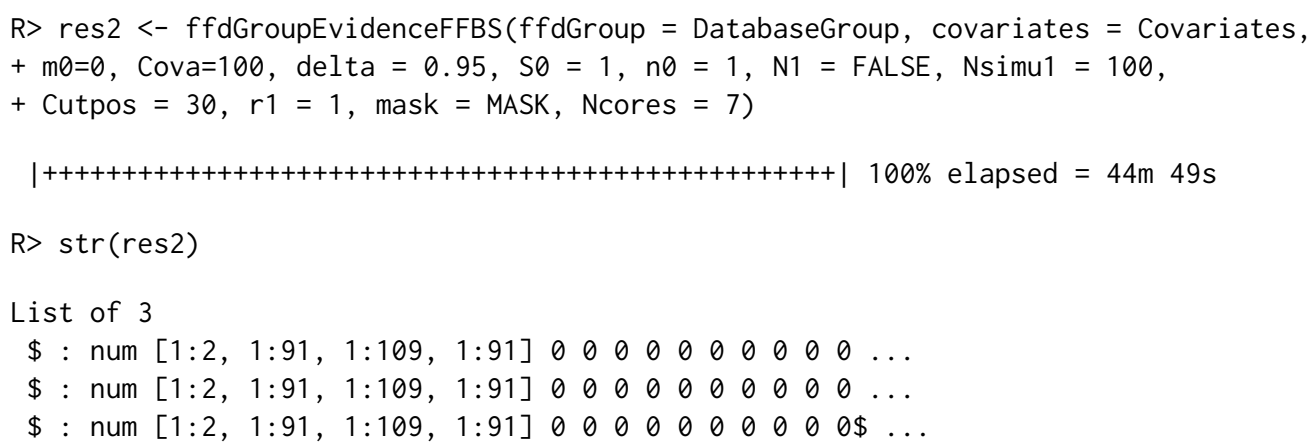

ffdGroupEvidenceFFBS returns an 3D array with the same structure and characteristics as its individual counterpart.

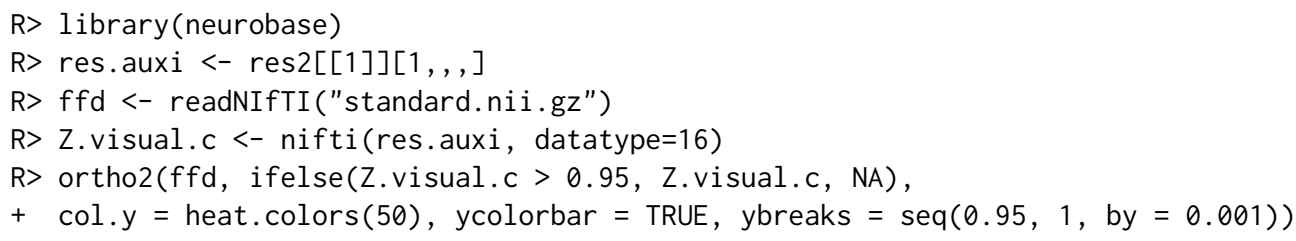



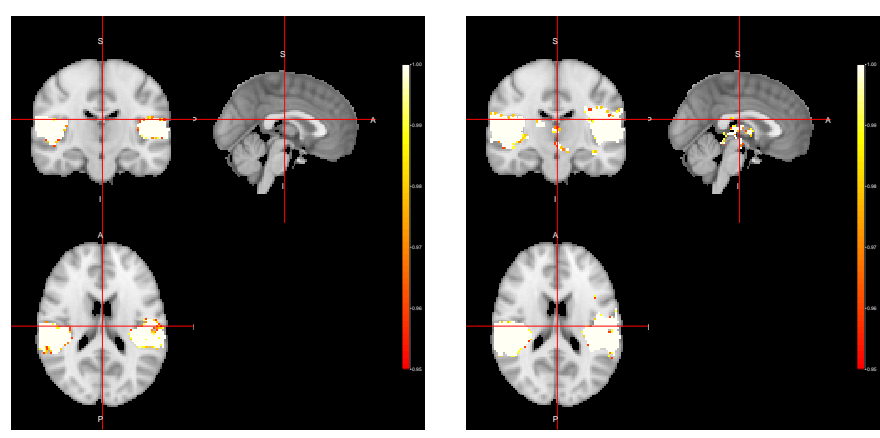

Figure 5: Activations maps obtained with the functions ffdGroupEvidenceFEST (left) and ffdGroupEvidenceFFBS (right) using the "Joint" as sampler distribution.

The functioning of the functions for single-voxel analysis at the group stage is the same as their counterparts at the individual stage.

R> resSingle <- GroupSingleVoxelFEST(posi.ffd $=c(14,56,40)$, DatabaseGroup,

+ covariates $=$ Covariates, $\mathrm{m} \theta=0$, Cova $=100$, delta $=0.95, \mathrm{~s} 0=1, \mathrm{n} \theta=1$,

$+\mathrm{N} 1=$ FALSE, Nsimu1 $=100, r 1=1$, Test = "Joint", Cutpos = 30)

$R>$ frame()

$\mathrm{R}>$ plot.window $(x \lim =c(30,311), y \lim =c(-5,5))$

$\operatorname{axis}(1, \operatorname{at}=\operatorname{seq}(30,310$, by $=20), \quad l w d=2, x l a b=$ "Time")

$\mathrm{R}>$ box $(1 w d=2)$

$\mathrm{R}>\operatorname{for}(\mathrm{j}$ in $2: 7)\{$ for ( $\mathrm{i}$ in $1: \operatorname{dim}(\operatorname{resSingle}[[4]])[1])\{\operatorname{lines}(31: \operatorname{dim}($ covariates)[1],

$+\operatorname{resSingle}[[4]][i, j]$, col $=$ "green") $\}\}$

$\mathrm{R}>$ for (i in $1: \operatorname{dim}(\operatorname{resSingle[}[4]])[1])\{\operatorname{lines}(31: \operatorname{dim}($ covariates)[1],

+ resSingle[[4]][i, 1,], col = "black")\}

lines(31:dim(covariates)[1], covariates[31:dim(covariates)[1], 1],

$+\operatorname{col}=$ "red", lwd = 2)

posi.ffd $=c(14,56,40)$

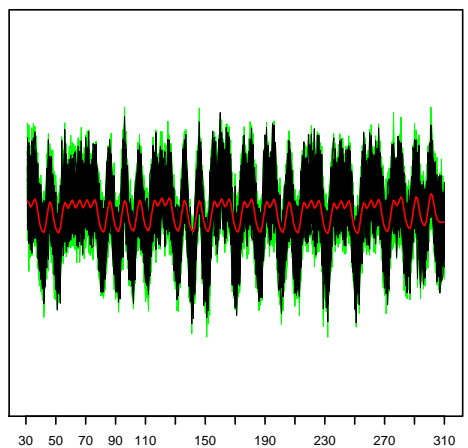

posi.ffd $=c(28,67,15)$

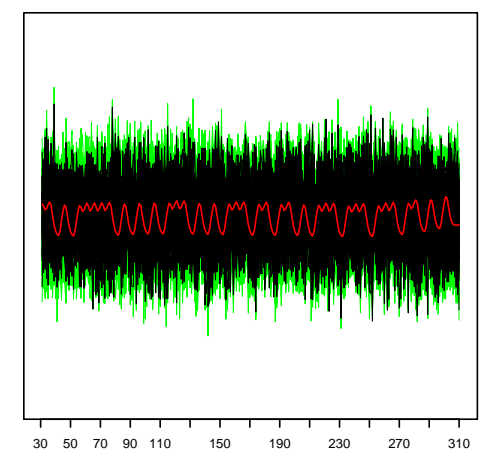

Figure 6: The black curves are simulated BOLD responses for voxel at position posi. $\mathrm{ffd}$ and the green curves are the BOLD responses related to its neighbors. The red curve is the expected BOLD response for the "voice localizer" experiment.

$\mathrm{R}>$ frame ()

$\mathrm{R}>$ plot.window $(x \lim =c(30,311), y \lim =c(-0.5,4.5), y l a b=\operatorname{expression}($ theta $))$

$\mathrm{R}>\operatorname{axis}(1, \operatorname{at}=\operatorname{seq}(30,310$, by $=20), \quad l w d=2, x l a b=$ "Time") 


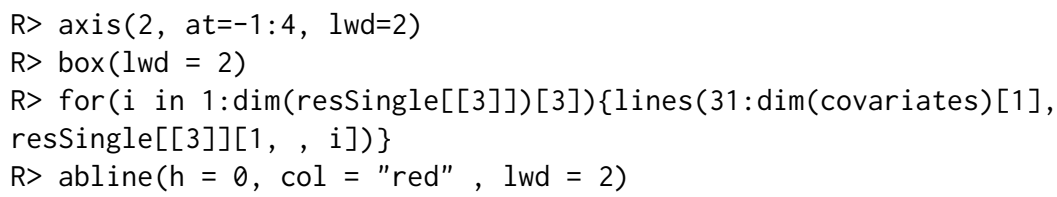

posi.ffd $=c(14,56,40)$

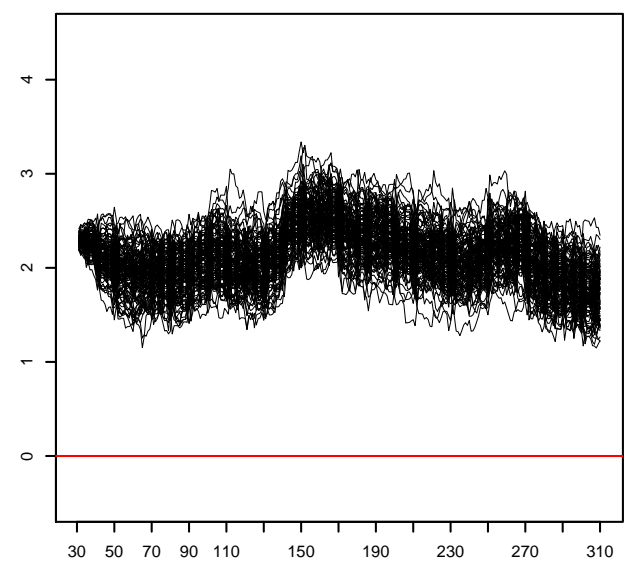

posi.ffd $=c(28,67,15)$

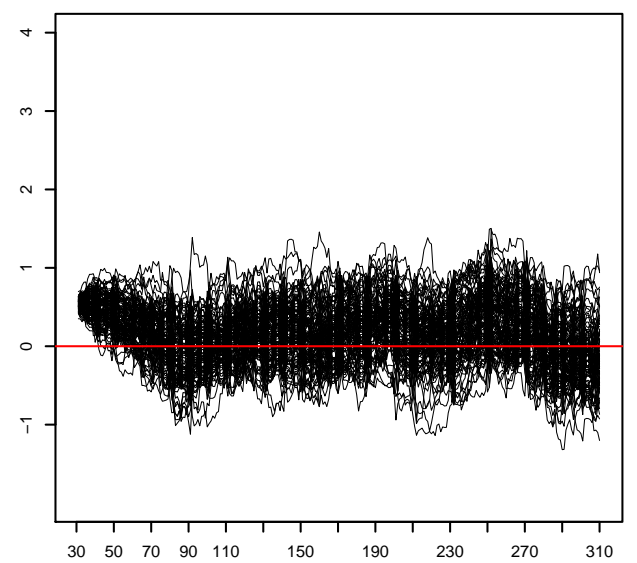

Figure 7: Simulated online trajectories of the state parameter for an active (left) and nonactive (right) volxel respectively.

\section{Conclusions and future work}

In this work, we present the BayesDLMfMRI package, which allows performing statistical analysis for fMRI data at individual and group stages. It offers different options to assess brain activation for single voxels as well as the entire brain volume and/or more specific brain regions with the help of a mask. The low-level functions are written in $\mathrm{C}++$ and options for parallel computation are available in some of the functions. Currently, some extensions for this package related to comparisons between groups and comparisons between tasks as well as other relevant features are being developed.

\section{Computational details}

The results in this paper were obtained using $\mathbf{R} 3.4 .4$ with the $\mathbf{R c p p}$ 1.0.2, RcppArmadillo 0.9.200.5.0 and pbapply 1.4.2 packages on a computer with Linux-Ubuntu, 32 CPUs and 188GB of RAM. R itself and all packages used are available from the Comprehensive $\mathbf{R}$ Archive Network (CRAN) at https://CRAN. R-project.org/.

\section{Bibliography}

M. Bezener, J. Hughes, G. Jones, et al. Bayesian spatiotemporal modeling using hierarchical spatial priors, with applications to functional magnetic resonance imaging. Bayesian Analysis, 2018. [p1]

M. Brett, I. S. Johnsrude, and A. M. Owen. The problem of functional localization in the human brain. Nature reviews neuroscience, 3(3):243, 2002. [p4]

A. F. da Silva et al. cudabayesreg: parallel implementation of a bayesian multilevel model for fmri data analysis. Journal of Statistical Software, 44(4):1-24, 2011. [p1] 
D. Eddelbuettel and C. Sanderson. Rcpparmadillo: Accelerating r with high-performance c++ linear algebra. Computational Statistics and Data Analysis, 71:1054-1063, March 2014. URL http://dx. doi. org/10.1016/j.csda.2013.02.005. [p1]

D. Eddelbuettel, R. François, J. Allaire, K. Ushey, Q. Kou, N. Russel, J. Chambers, and D. Bates. Rcpp: Seamless $r$ and c++ integration. Journal of Statistical Software, 40(8):1-18, 2011. [p1]

A. Eklund, M. A. Lindquist, and M. Villani. A bayesian heteroscedastic glm with application to fmri data with motion spikes. NeuroImage, 155:354-369, 2017. [p1]

K. Gorgolewski, O. Esteban, G. Schaefer, B. Wandell, and R. Poldrack. Openneuro - a free online platform for sharing and analysis of neuroimaging data. Organization for Human Brain Mapping. Vancouver, Canada, 1677, 2017. [p3]

M. Jenkinson, C. F. Beckmann, T. E. Behrens, M. W. Woolrich, and S. M. Smith. Fsl. Neuroimage, 62(2): 782-790, 2012. [p1]

J. C. Jiménez. fmri group analysis based on outputs from matrix-variate dynamic linear models. arXiv:1911.00708, 2019. [p1,3]

J. C. Jiménez, C. A. de B. Pereira, and V. Fossaluza. Assessing dynamic effects on a bayesian matrixvariate dynamic linear model: an application to fmri data analysis. arXiv:1910.12058, 2019. [p1, 2]

J. H. Kook, M. Guindani, L. Zhang, and M. Vannucci. Npbayes-fmri: Non-parametric bayesian general linear models for single-and multi-subject fmri data. Statistics in Biosciences, 11(1):3-21, 2019. [p1]

J. Muschelli. neurobase: 'Neuroconductor' Base Package with Helper Functions for 'nifti' Objects, 2018. URL https://CRAN.R-project.org/package=neurobase. R package version 1.27.6. [p1]

W. D. Penny, K. J. Friston, J. T. Ashburner, S. J. Kiebel, and T. E. Nichols. Statistical parametric mapping: the analysis of functional brain images. Elsevier, 2011. [p1]

C. R. Pernet, P. McAleer, M. Latinus, K. J. Gorgolewski, I. Charest, P. E. Bestelmeyer, R. H. Watson, D. Fleming, F. Crabbe, M. Valdes-Sosa, et al. The human voice areas: Spatial organization and inter-individual variability in temporal and extra-temporal cortices. Neuroimage, 119:164-174, 2015. [p3]

J. M. Quintana. A dynamic linear matrix-variate regression model, 1985. [p1,2]

J. M. Quintana. Multivariate Bayesian forecasting models. PhD thesis, University of Warwick, 1987. [p2]

J. M. Quintana and M. West. An analysis of international exchange rates using multivariate dlm's. The Statistician, pages 275-281, 1987. [p4]

R Core Team. R: A Language and Environment for Statistical Computing. R Foundation for Statistical Computing, Vienna, Austria, 2018. URL https://www.R-project.org/. [p1]

N. Sanyal and M. A. Ferreira. BHMSMAfMRI: Bayesian Hierarchical Multi-Subject Multiscale Analysis of Functional MRI Data, 2019. URL https: //CRAN. R-project.org/package=BHMSMAfMRI. R package version 1.3. $[\mathrm{p} 1]$

P. Solymos and Z. Zawadzki. pbapply: Adding Progress Bar to '*apply' Functions, 2019. URL https: //CRAN. R-project. org/package=pbapply. R package version 1.4-1. [p1]

K. Tabelow and J. Polzehl. Statistical parametric maps for functional mri experiments in R: The package fmri. Journal of Statistical Software, 44(11):1-21, 2011. URL http://www. jstatsoft. org/v44/i11/. [p1]

M. Welvaert, J. Durnez, B. Moerkerke, G. Verdoolaege, and Y. Rosseel. neurosim: An r package for generating fmri data. Journal of Statistical Software, 44(10):1-18, 2011. [p1]

B. Whitcher, V. J. Schmid, and A. Thornton. Working with the DICOM and NIfTI data standards in R. Journal of Statistical Software, 44(6):1-28, 2011. URL http://www. jstatsoft.org/v44/i06/. [p1]

C.-H. Yu, R. Prado, H. Ombao, and D. Rowe. A bayesian variable selection approach yields improved detection of brain activation from complex-valued fmri. Journal of the American Statistical Association, (just-accepted):1-61, 2018. [p1]

L. Zhang, M. Guindani, and M. Vannucci. Bayesian models for fmri data analysis. Wiley interdisciplinary reviews. Computational statistics, $7(1): 21-41,2015$. [p1] 
L. Zhang, M. Guindani, F. Versace, J. M. Engelmann, M. Vannucci, et al. A spatiotemporal nonparametric bayesian model of multi-subject fmri data. The Annals of Applied Statistics, 10(2):638-666, 2016. [p1] 


\section{References}

Beckmann, C. F., Jenkinson, M., \& Smith, S. M. (2003). General multilevel linear modeling for group analysis in fmri. Neuroimage, 20(2), 1052-1063.

1

Bezener, M., Hughes, J., Jones, G., et al. (2018). Bayesian spatiotemporal modeling using hierarchical spatial priors, with applications to functional magnetic resonance imaging. Bayesian Analysis.

1

Bowman, F. D. (2014). Brain imaging analysis. Annual review of statistics and its application, 1, $61-85$.

1

Eklund, A., Andersson, M., Josephson, C., Johannesson, M., \& Knutsson, H. (2012). Does parametric fmri analysis with spm yield valid results? - an empirical study of 1484 rest datasets. NeuroImage, 61(3), 565-578.

1,7

Eklund, A., Lindquist, M. A., \& Villani, M. (2017). A bayesian heteroscedastic glm with application to fmri data with motion spikes. NeuroImage, 155, 354-369. 1

Eklund, A., Nichols, T. E., \& Knutsson, H. (2016). Cluster failure: why fmri inferences for spatial extent have inflated false-positive rates. Proceedings of the National Academy of Sciences, 113(28), 7900-7905.

1,7

Jenkinson, M., Beckmann, C. F., Behrens, T. E., Woolrich, M. W., \& Smith, S. M. (2012). Fsl. Neuroimage, 62(2), 782-790.

1

Kashou, N. H. (2014). A practical guide to an fmri experiment. in: Duric d, editor. advanced brain neuroimaging topics in health and disease - methods and applications. 3

Penny, W. D., Friston, K. J., Ashburner, J. T., Kiebel, S. J., \& Nichols, T. E. (2011). Statistical parametric mapping: the analysis of functional brain images. Elsevier.

1

Pernet, C. R., McAleer, P., Latinus, M., Gorgolewski, K. J., Charest, I., Bestelmeyer, P. E., ... others (2015). The human voice areas: Spatial organization and inter-individual variability in temporal and extra-temporal cortices. Neuroimage, 119, 164-174.

1

Poldrack, R. A., Mumford, J. A., \& Nichols, T. E. (2011). Handbook of functional mri data analysis. Cambridge University Press.

$2,4,5,6$ 
Quintana, J. M. (1985). A dynamic linear matrix-variate regression model.

1

West, M., \& Harrison, J. (1997). Bayesian forecasting and dynamic models (2nd ed.). New York, NY, USA: Springer-Verlag New York, Inc.

1

Worsley, K. J., \& Friston, K. J. (1995). Analysis of fmri time-series revisited-again. Neuroimage, 2(3), 173-181.

6

Yu, C.-H., Prado, R., Ombao, H., \& Rowe, D. (2018). A bayesian variable selection approach yields improved detection of brain activation from complex-valued fmri. Journal of the American Statistical Association(just-accepted), 1-61.

1

Zhang, L., Guindani, M., \& Vannucci, M. (2015). Bayesian models for fmri data analysis. Wiley interdisciplinary reviews. Computational statistics, 7(1), 21-41.

1

Zhang, L., Guindani, M., Versace, F., Engelmann, J. M., Vannucci, M., et al. (2016). A spatiotemporal nonparametric bayesian model of multi-subject fmri data. The Annals of Applied Statistics, $10(2), 638-666$.

1 